Issued by Sandia National Laboratories, operated for the United States Department of Energy by Sandia Corporation.

NOTICE: This report was prepared as an account of work sponsored by an agency of the United States Government. Neither the United States Government, nor any agency thereof, nor any of their employees, nor any of their contractors, subcontractors, or their employees, make any warranty, express or implied, or assume any legal liability or responsibility for the accuracy, completeness, or usefulness of any information, apparatus, product, or process disclosed, or represent that its use would not infringe privately owned rights. Reference herein to any specific commercial product, process, or service by trade name, trademark, manufacturer, or otherwise, does not necessarily constitute or imply its endorsement, recommendation, or favoring by the United States Government, any agency thereof, or any of their contractors or subcontractors. The views and opinions expressed herein do not necessarily state or reflect those of the United States Government, any agency thereof, or any of their contractors.

Printed in the United States of America. This report has been reproduced directly from the best available copy.

Available to DOE and DOE contractors from Office of Scientific and Technical Information

P.O. Box 62

Oak Ridge, TN 37831

Prices available from (703) 605-6000

Web site: http://www.ntis.gov/ordering.htm

Available to the public from

National Technical Information Service

U.S. Department of Commerce

5285 Port Royal Rd

Springfield, VA 22161

NTIS price codes

Printed copy: A05

Microfiche copy: AO1

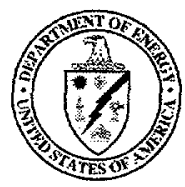




\section{DISCLAIMER}

Portions of this document may be illegible in electronic image products. Images are produced from the best available original document. 
SAND \#99-1539

Unlimited Release

Printed June 1999

\title{
Particle Transport in Parallel-Plate Reactors
}

\author{
D. J. Rader and A. S. Geller \\ Plasma and Aerosol Sciences Department \\ Sandia National Laboratories \\ P. O Box 5800 \\ Albuquerque, New Mexico 87185-0827
}

\begin{abstract}
A major cause of semiconductor yield degradation is contaminant particles that deposit on wafers while they reside in processing tools during integrated circuit manufacturing. This report presents numerical models for assessing particle transport and deposition in a parallel-plate geometry characteristic of a wide range of single-wafer processing tools: uniform downward flow exiting a perforatedplate showerhead separated by a gap from a circular wafer resting on a parallel susceptor. Particles are assumed to originate either upstream of the showerhead or from a specified position between the plates. The physical mechanisms controlling particle deposition and transport (inertia, diffusion, fluid drag, and external forces) are reviewed, with an emphasis on conditions encountered in semiconductor process tools (i.e., sub-atmospheric pressures and submicron particles). Isothermal flow is assumed, although small temperature differences are allowed to drive particle thermophoresis. Numerical solutions of the flow field are presented which agree with an analytic, creeping-flow expression for $\operatorname{Re}<4$. Deposition is quantified by use of a particle collection efficiency, which is defined as the fraction of particles in the reactor that deposit on the wafer. Analytic expressions for collection efficiency are presented for the limiting case where external forces control deposition (i.e., neglecting particle diffusion and inertia). Deposition from simultaneous particle diffusion and external forces is analyzed by an Eulerian formulation; for creeping flow and particles released from a planar trap, the analysis yields an analytic, integral expression for particle deposition based on process and particle properties. Deposition from simultaneous particle inertia and external forces is analyzed by a Lagrangian formulation, which can describe inertia-enhanced deposition resulting from particle acceleration in the showerhead. An approximate analytic expression is derived for particle velocity at the showerhead exit as a function of showerhead geometry, flow rate, and gas and particle properties. The particle showerhead-exit velocity is next used as an initial condition for particle transport between the plates to determine whether the particle deposits on the wafer, as a function of showerhead-exit particle velocity, the plate separation, flow rate, and gas and particle properties. Based on the numerical analysis, recommendations of best practices are presented that should help tool operators and designers reduce particle deposition in real tools. These guidelines are not intended to replace detailed calculations, but to provide the user with a general feel for inherently-clean practices.
\end{abstract}




\section{Acknowledgment}

The authors gratefully acknowledge interactions with Seung Choi, John Brockmann, Scott Sibbett, and Gene Feit. This work was performed at Sandia National Laboratories' Contamination Free Manufacturing Research Center under the joint support of the United States Department of Energy and SEMATECH. 


\section{Table of Contents}

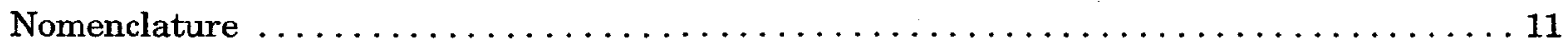

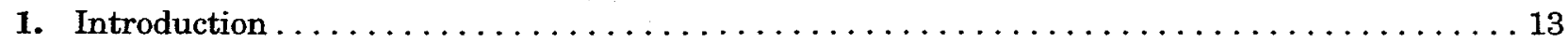

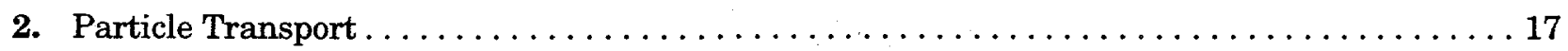

2.1 Noncontinuum Considerations . . . . . . . . . . . . . . . . . . 17

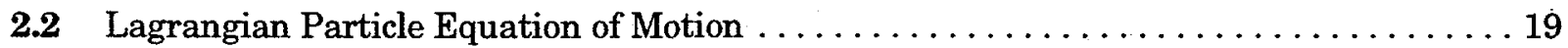

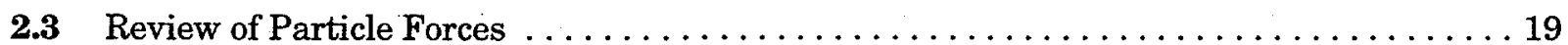

2.3.1 Fluid-Particle Drag Force. . . . . . . . . . . . . . . . . . . . 19

2.3.2 Gravitational Force. . . . . . . . . . . . . . . . . . . . . . 22

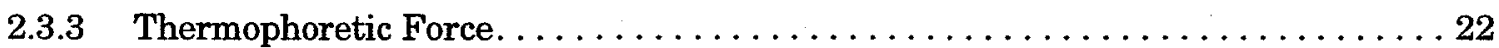

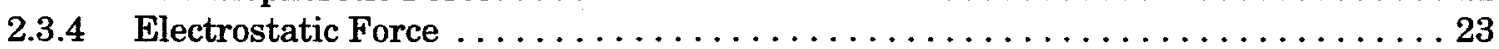

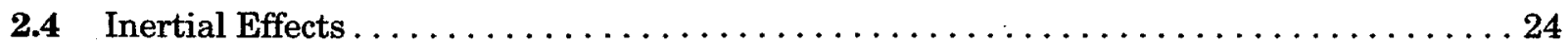

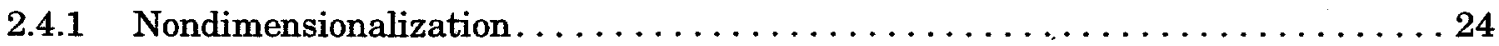

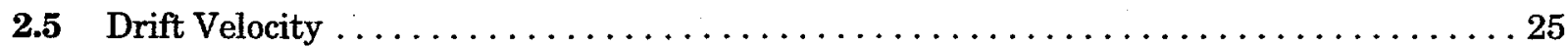

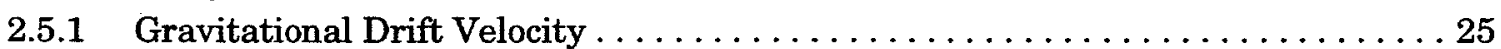

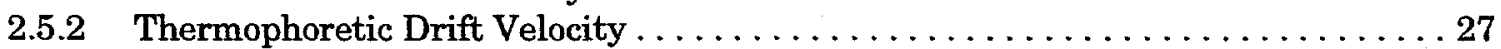

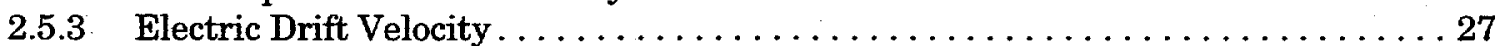

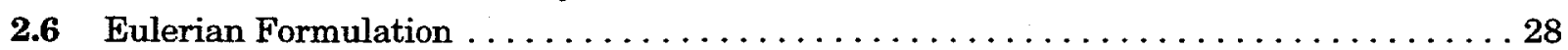

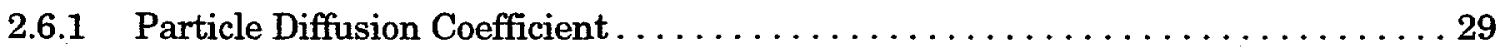

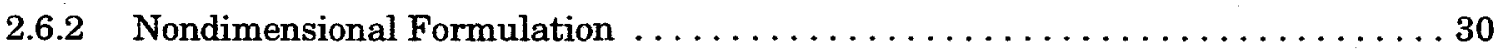

3. Analysis of Fluid Flow in a Parallel Plate Reactor $\ldots \ldots \ldots \ldots \ldots \ldots \ldots \ldots \ldots \ldots \ldots \ldots \ldots$

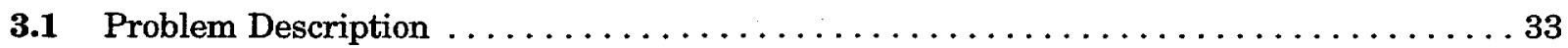

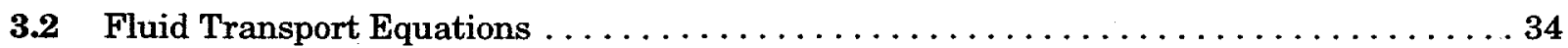

3.3 Fluid Transport in the Showerhead Holes ... . . . . . . . . . . . . . . 35

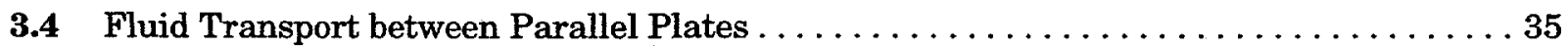

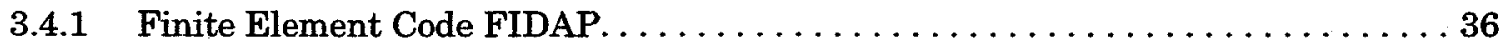

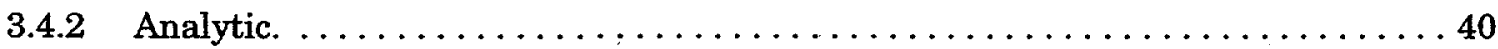

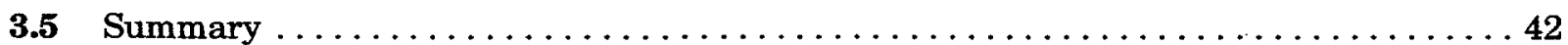

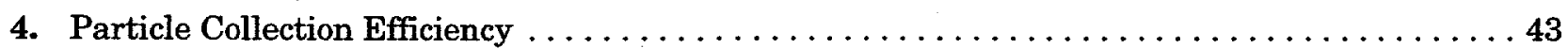

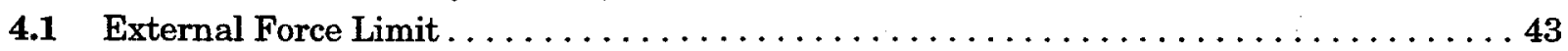

4.2 Particles Entering Through the Showerhead. . $\ldots \ldots \ldots \ldots \ldots \ldots \ldots \ldots \ldots \ldots 4$

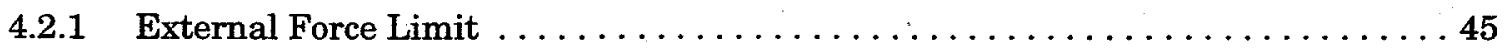

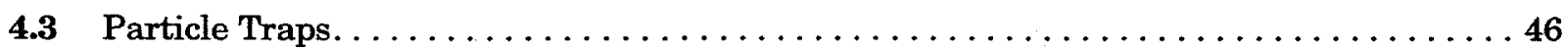

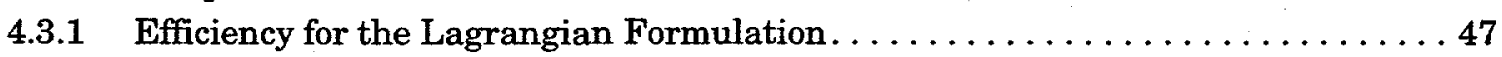

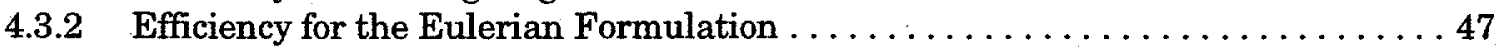

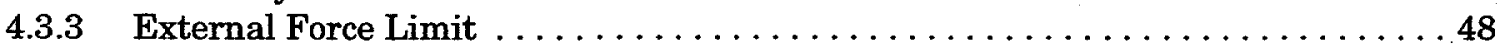

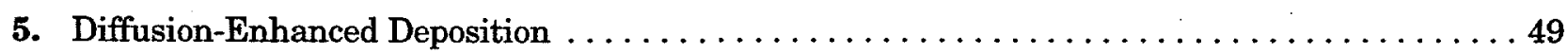

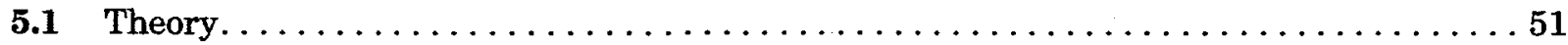

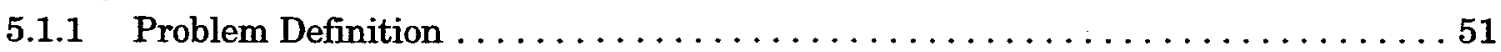

5.1.2 Solution of the Eulerian Particle Transport Equation . . . . . . . . . . . 51

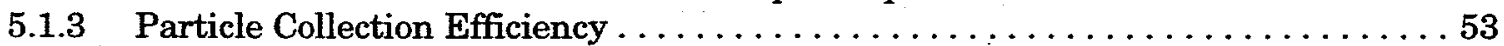

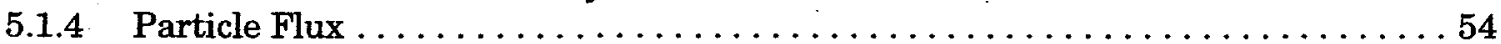

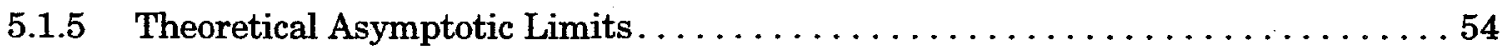

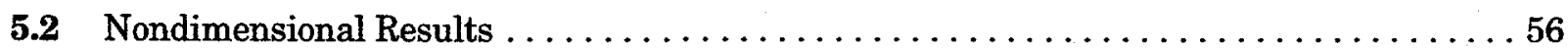

5.2.1 Comparison of Calculated Results to Asymptotic Limits . . . . . . . . . . . . 58

5.2 .2 Efficiency at Intermediate Peclet Numbers $\ldots \ldots \ldots \ldots \ldots \ldots \ldots \ldots \ldots$

5.2.3 Linear Combination of Deposition Terms ................... 60 


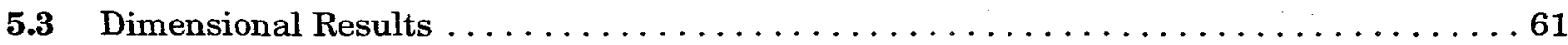

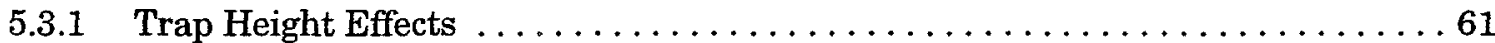

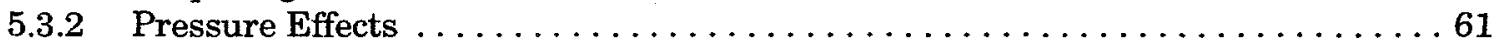

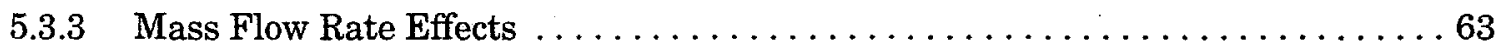

5.3.4 Effect of Thermophoresis . . . . . . . . . . . . . . . . . . . . 63

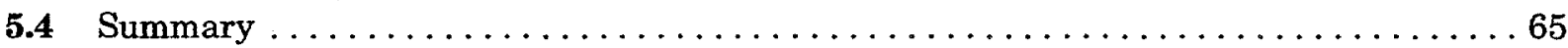

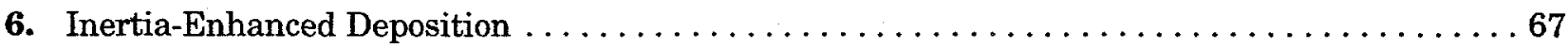

6.1 Particle Transport in the Showerhead Holes....................... 68

6.2 Particle Transport between Parallel Plates . . . . . . . . . . . . . . . . . 70

6.2 .1 General Results. . . . . . . . . . . . . . . . . . . . . . . . . 70

6.2.2 Comparison of Efficiencies using Numerical and Analytic Flow Solutions. . . . . . 71

6.2.3 Effect of Fluid Reynolds Number. . . . . . . . . . . . . . . . . . . . 74

6.2.4 Asymptotic Limit of Critical Stokes Number . . . . . . . . . . . . . . . . .75

6.3 Coupled Transport - Nondimensional Results . . . . . . . . . . . . . . . 77

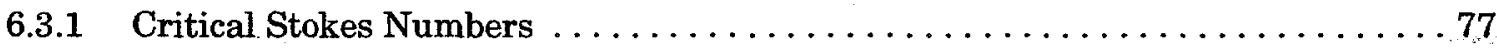

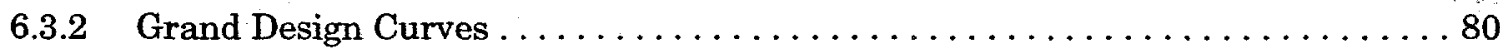

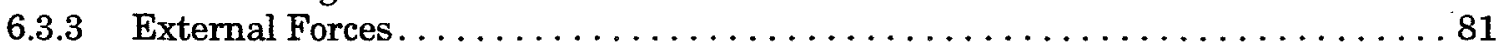

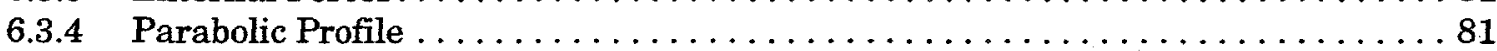

6.4 Coupled Transport - Dimensional Results $\ldots \ldots \ldots \ldots \ldots \ldots \ldots \ldots \ldots \ldots \ldots \ldots . \ldots \ldots$

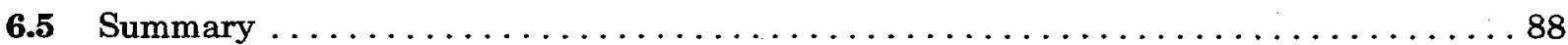

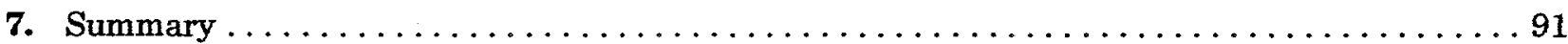

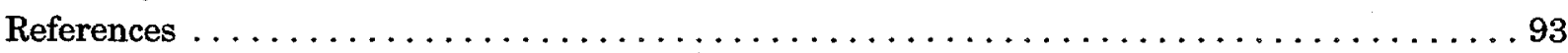

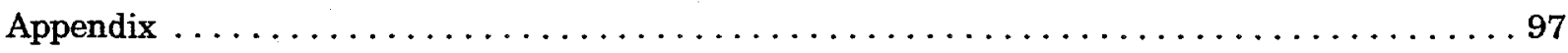

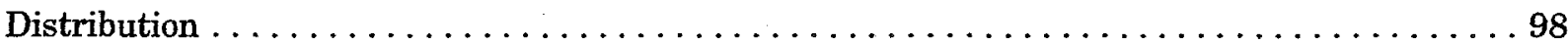




\section{List of Figures}

Figure 1. Parallel-plate Reactor Geometry. Schematic of the reactor geometry assumed in this work: a) top view of a showerhead and $b$ ) side view of a parallel-plate reactor. . . . . . 15

Figure 2. Slip Correction Factor. Dependence of the slip correction factor on particle Knudsen number (solid curve). The free molecular and continuum limits for the slip correction factor

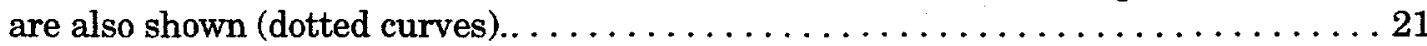

Figure 3. Gravitational Drift Velocity. Dependence of the gravitational drift (or settling) velocity on particle diameter for six process pressures (argon at $293 \mathrm{~K}$, particle density $\rho_{\mathrm{p}}=1 \mathrm{~g} / \mathrm{cm}^{3}$ ). 26

Figure 4. Thermophoretic Drift Velocity. Dependence of the thermophoretic drift velocity on particle diameter for six process pressures (argon at $293 \mathrm{~K}, k_{\mathrm{g}} / k_{\mathrm{p}}=0.001, \nabla T=1 \mathrm{~K} / \mathrm{cm}$ ). . . 28 28

Figure 5. Diffusion Coefficient. Dependence of the particle diffusion coefficient on particle diameter

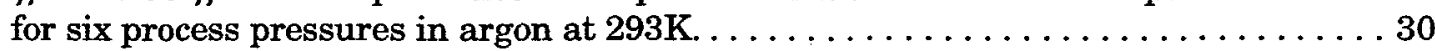

Figure 6. Grid Refinement Studies. Axial and radial velocity profiles for $R e=1,4$, and 8 calculated on a coarse ( 15 elements) and refined ( 30 elements) mesh. Lines represent the results of the refined mesh, while the symbols give the results of the coarse mesh. ( $r=S=1$ for all

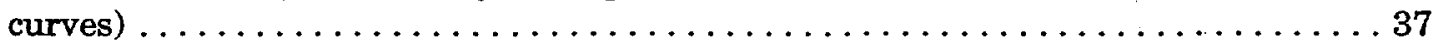

Figure 7. Flow Field Results for Various Re. Axial and radial velocity profiles for $R e=0,1,2,4$, and 8 calculated on a refined (30 elements) mesh. $(r / S=1$ for all curves) $\ldots \ldots \ldots \ldots 38$

Figure 8. Edge Effects. Axial velocity at the mid-plane $(z / S=0.5)$ is plotted versus radius for gap spacings $S=1,4$, and 8 at $R e=8$, for $S=1$ at $R e=4$, and for $S=1$ and 8 at $R e=1$ ( $30 \times 60$ element

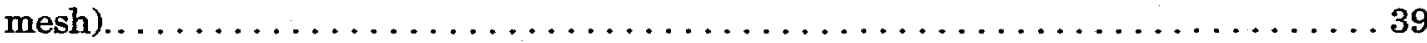

Figure 9. Comparison of 1- and 2-D Solutions. The ratio of the 1-D analytic to the 2-D FIDAP numerical solution are shown for both the axial and radial velocity. Results for $\operatorname{Re}=1,2,4,6$, and 8 are shown. The FIDAP solution used a refined mesh ( $30 \times 60$ element) with a gap spacing $S=1$; the profile was taken at $r=1$, where edge effects are small. . . . . 41

Figure 10. Critical Trajectory. Diagram of a critical trajectory for a particle which starts at the showerhead at radial position $R_{\text {crit }}$ and deposits at the wafer edge $R_{\mathrm{W}} \ldots \ldots \ldots \ldots \ldots 4$

Figure 11. Trap Schematic. Diagram of particles in a planar trap located a distance $h$ from the lower plate; a critical trajectory is also shown for a particle which starts at radial position $R_{\text {crit }}$

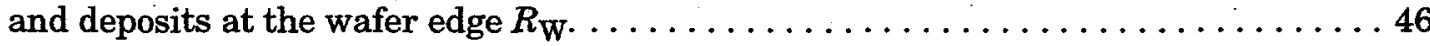

Figure 12. Geometry. Diagram of parallel, infinite-plate geometry with particles in a planar trap located a distance $h$ from the lower plate. . . . . . . . . . . . . . . . 49

Figure 13. Efficiency vs. Pe for Various Drift Velocities. Figures show calculated efficiencies for particles starting in traps at: a) $h / S=0.1$, b) $h / S=0.5$, and c) $h / S=0.9 \ldots \ldots \ldots \ldots 57$

Figure 14. Efficiency vs. Pe for Various Reynolds Numbers. Figures show calculated efficiencies for

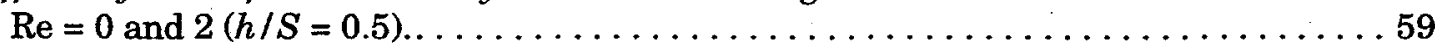

Figure 15. Efficiency vs. particle diameter and trap position (isothermal case). Collection efficiencies for particle transport over a $200 \mathrm{~mm}$ wafer including gravitational settling and diffusion for dimensionless trap locations of $0.1,0.3,0.5,0.7$, and $0.9(S=2.54 \mathrm{~cm}, Q=1000 \mathrm{sccm}$ of argon, $P=1$ torr, $T=300 \mathrm{~K}$, $R e=0.98$, and particle density of $1 \mathrm{~g} / \mathrm{cc}) \ldots \ldots \ldots \ldots \ldots 2$ 
Figure 16. Efficiency vs. particle diameter and pressure (isothermal case). Collection efficiencies for particle transport over a $200 \mathrm{~mm}$ wafer including gravitational settling and diffusion for reactor pressures of $1,10,100$, and 760 torr $(S=2.54 \mathrm{~cm}, Q=1000 \mathrm{sccm}$ of argon, $h=1.27 \mathrm{~cm}, T=300 \mathrm{~K}, \mathrm{Re}=0.98$, and particle density of $1 \mathrm{~g} / \mathrm{cc}) \ldots \ldots \ldots \ldots \ldots \ldots \ldots \ldots \ldots \ldots$

Figure 17. Efficiency vs. particle diameter and flow rate (isothermal case). Collection efficiencies for particle transport over a $200 \mathrm{~mm}$ wafer including gravitational settling and diffusion for gas mass flow rates of 10,100 , and $1000 \mathrm{sccm}$ (argon, $S=2.54 \mathrm{~cm}, h=1.27 \mathrm{~cm}, T=300 \mathrm{~K}$, $P=1$ torr, $0.01<\operatorname{Re}<0.98$, and particle density of $1 \mathrm{~g} / \mathrm{cc}) \ldots \ldots \ldots \ldots \ldots \ldots$

Figure 18. Efficiency vs. particle diameter and wafer temperature. Collection efficiencies including gravity, thermophoresis, and diffusion for wafer temperatures of $280,290,300,310$, and $320 \mathrm{~K}\left(200 \mathrm{~mm}\right.$ wafer, $1000 \mathrm{sccm}$ argon, $S=2.54 \mathrm{~cm}, h=1.27 \mathrm{~cm}, T_{\text {showerhead }}=300 \mathrm{~K}$,

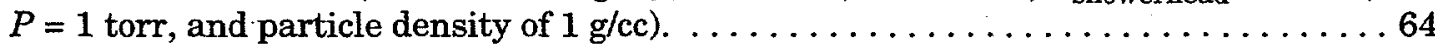

Figure 19. Acceleration of Particles Through Showerhead. Dimensionless velocity of particles exiting showerhead tubes, $V_{\mathrm{po}} / U_{\text {jet }}$, as a function of jet Stokes number, $\mathrm{St}_{\text {jet }}$, for a range of veloc-

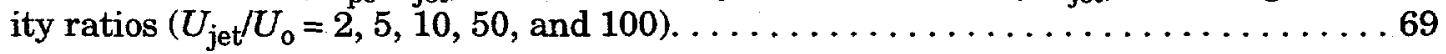

Figure 20. Efficiency vs. Stokes number for Various Drift Velocities for Re=8. Solid lines are calculated using an analytic flow field and a Runge-Kutta integrator, while the symbols are calculated using numerical flow solutions and the FIDAP particle tracking post-processing routines. a) Particle dimensionless initial velocity of $-1, b$ ) Particle dimensionless initial

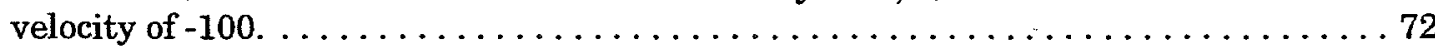

Figure 21. Efficiency vs. Stokes number for Various Drift Velocities and Reynolds Numbers. The initial particle velocity is $\mathbf{- 1}$ for all curves, which are calculated using the analytic flow field.75

Figure 22. Critical Stokes Number vs. Particle Dimensionless Inlet Velocity. Values of the critical Stokes number were calculated using the analytic approximation to the flow field for Reynolds numbers of 0,4 , and 8 . One set of curves applies for no external force $\left(V_{p}^{t} /\right.$ $U o=0)$, the other set applies for a strong force resisting deposition $\left(V_{\mathrm{p}}^{\mathrm{t}} / U o=1\right) \ldots \ldots 76$

Figure 23. Effect of Jet to Face-Velocity Ratio. Efficiency versus Stokes number for $U_{\text {jet }} / U_{0}=1,10,100$, and 1000 including coupling between showerhead and inter-plate transport (for this cal-

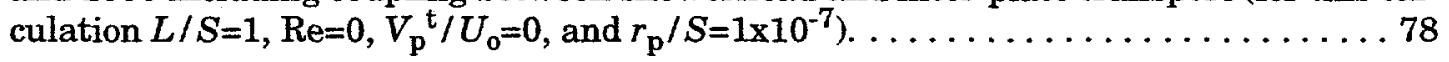

Figure 24. Effect of Showerhead Thickness. Efficiency versus Stokes number for $L / S=0.1,0.5,1$, and 2 including coupling between showerhead and inter-plate transport (for this calculation $U_{\text {jet }} / U_{0}=100, \operatorname{Re}=0, V_{\mathrm{p}}^{\mathrm{t}} / U_{0}=0$, and $\left.r_{\mathrm{p}} / S=1 \times 10^{-7}\right) \ldots \ldots \ldots \ldots \ldots \ldots \ldots \ldots \ldots \ldots \ldots \ldots \ldots \ldots \ldots$

Figure 25. Critical Stokes Number vs. Jet to Face-Velocity Ratio. Values of the critical Stokes number were calculated vs. jet to face-velocity ratios for various levels of resistive forces $\left(V_{p}^{t} / U_{0}\right.$ $=0,0.1$, and 1 ) including coupling between showerhead and inter-plate transport (for this

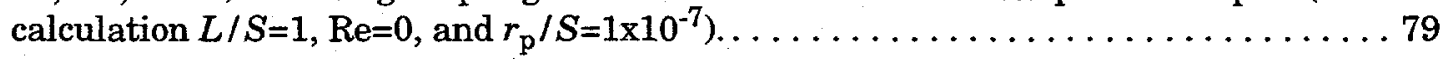

Figure 26. Grand Design Curve. Grand design curve for estimating the critical Stokes number based on showerhead parameters (for this calculation $\mathrm{Re}=0, V_{\mathrm{p}}^{\mathrm{t}} / U_{\mathrm{o}}=0$, and $r_{\mathrm{p}} / S=1 \times 10^{-7}$ )..80

Figure 27. Efficiency vs. Stokes for various drift velocities. Collection efficiencies for fully coupled particle transport for $V_{\mathrm{p}}^{\mathrm{t}} / U_{\mathrm{o}}$ values of $-0.5,-0.1,-0.01,0$, and 1 (for this calculation $\mathrm{Re}=0, L /$

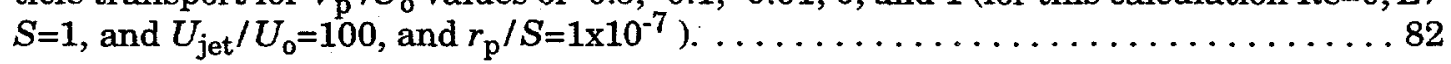

Figure 28. Efficiency vs. Stokes for parabolic showerhead profile (no external force). Collection efficiencies for fully coupled particle transport for particles experiencing the showerhead hole centerline and mean velocities (plug flow assumption), and integrated over the parabolic 
velocity profile in the showerhead holes in the absence of external forces ( $\operatorname{Re}=0, L / S=1$,

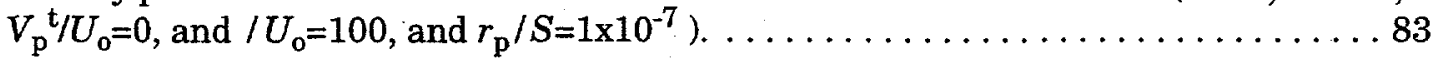

Figure 29. Efficiency us. Stokes for parabolic showerhead profile (attractive force). Collection efficiencies for fully coupled particle transport for particles experiencing the showerhead hole centerline and mean velocities (plug flow assumption), and integrated over the parabolic velocity profile in the showerhead holes for the case of a attractive force $(\operatorname{Re}=0, L / S=1$,

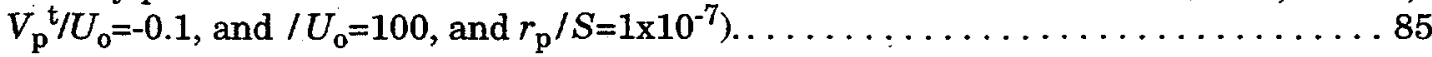

Figure 30. Efficiency vs. particle diameter and pressure (isothermal case). Collection efficiencies for fully coupled particle transport assuming plug flow through the showerhead hole for reactor pressures of $0.5,1.0,2.0$, and 10. torr $\left(\operatorname{Re}=0.98, L=S=2.54 \mathrm{~cm}, / U_{0}=99.2, Q=1000\right.$ sccm argon, $T=300 \mathrm{~K}$, and particle density of $1 \mathrm{~g} / \mathrm{cc}) \ldots \ldots \ldots \ldots \ldots \ldots \ldots \ldots \ldots$

Figure 31. Efficiency vs, particle diameter and wafer temperature (with thermophoresis). Collection efficiencies for fully coupled particle transport assuming plug flow through the showerhead hole for wafer temperatures of 280,300 , and $320 \mathrm{~K}$ ( $R e=0.98, L=S=2.54 \mathrm{~cm}, / U_{0}=99.2$, $Q=1000 \mathrm{sccm}$ argon, $P=1$ torr, $T_{\text {showerhead }}=300 \mathrm{~K}$, and particle density of $\left.1 \mathrm{~g} / \mathrm{cc}\right) \ldots 87$ 


\section{List of Tables}

Table 1: Gas Properties. Density, viscosity, molecular weight, mean free path, and slip correction constants (for oil drops) for nine common gases at $T_{0}=296.15 \mathrm{~K}$ and $P_{0}=760$ torr (Rader, 1990). 18

Table 2: Comparison of particle collection efficiencies calculated using analytic and FIDAP flowfields. Various combinations of particle initial velocities $V_{\mathrm{po}} / U_{\mathrm{o}}$ and flow Reynolds numbers are presented for the case where no external force is acting $\left(V_{p}^{t} / U_{o}=0\right) \ldots \ldots \ldots \ldots \ldots 73$

Table 3: Tabulation of Grand Design Curve Data. Critical Stokes numbers for various dimensionless jet velocities and showerhead thicknesses $\left(\mathrm{Re}=0\right.$, no external force applied, $\left.r_{\mathrm{p}} / S=10^{-7}\right) \ldots 97$ 


\section{Nomenclature}

\begin{tabular}{|c|c|}
\hline$a_{j e t}$ & showerhead hole radius $(\mathrm{cm})$ \\
\hline$A_{j e t}$ & showerhead hole area $\left(\mathrm{cm}^{2}\right)$ \\
\hline$A_{\text {showerhead }}$ & showerhead (and wafer) area $\left(\mathrm{cm}^{2}\right)$ \\
\hline & gas mean molecular velocity $(\mathrm{cm} / \mathrm{s})=(8 R T / \pi M)^{1 / 2}$ \\
\hline$C_{m}$ & coefficient in thermophoretic force expression $(-)$ \\
\hline$C_{\mathrm{s}}$ & coefficient in thermophoretic force expression (-) \\
\hline$C_{t}$ & coefficient in thermophoretic force expression (-) \\
\hline$d_{j e t}$ & showerhead hole diameter $(\mathrm{cm})$ \\
\hline$d_{p}$ & particle diameter $(\mathrm{cm})$ \\
\hline $\mathscr{D}$ & Stokes-Einstein particle diffusion coefficient $\left(\mathrm{cm}^{2} / \mathrm{sec}\right)$ \\
\hline$D_{W}$ & wafer (and showerhead) diameter $(\mathrm{cm})$ \\
\hline$e^{n}$ & elementary electric charge $\left(4.803 \cdot 10^{-10} \mathrm{esu}\right)$ \\
\hline $\boldsymbol{E}$ & local electric field (dyne/esu) \\
\hline$F_{D}$ & drag force acting on particle (dyne) \\
\hline$F_{E}$ & electric force acting on particle (dyne) \\
\hline$F_{G}$ & gravitational force acting on particle (dyne) \\
\hline$F_{T}$ & thermophoretic force acting on particle (dyne) \\
\hline$g$ & gravitational acceleration $\left(980 \mathrm{~cm} \mathrm{~s}^{-2}\right)$ \\
\hline$h$ & particle trap/source distance from lower plate $(\mathrm{cm})$ \\
\hline$J_{o}$ & particle flux to wafer $\left(\# \mathrm{~cm}^{-2} \mathrm{~s}^{-1}\right)$ \\
\hline$k$ & Boltzmann constant $\left(1.380658 \cdot 10^{-16} \mathrm{erg} \mathrm{K}^{-1}\right)$ \\
\hline$k_{g}$ & gas thermal conductivity (erg cm $\mathrm{cm}^{-1} \mathrm{~s}^{-1} \mathrm{~K}^{-1}$ ) \\
\hline$k_{p}$ & particle thermal conductivity (erg $\mathrm{cm}^{-1} \mathrm{~s}^{-1} \mathrm{~K}^{-1}$ ) \\
\hline$\dot{K}_{T}$ & interpolating factor in thermophoretic force expression $(-)$ \\
\hline$L$ & showerhead thickness $(\mathrm{cm})$ \\
\hline$m_{p}$ & particle mass ( $\mathrm{g}$ ) \\
\hline$M$ & gas molecular weight $(\mathrm{g} / \mathrm{mol})$ \\
\hline$n$ & particle concentration $\left(\# / \mathrm{cm}^{3}\right)$ \\
\hline$n_{p}$ & number of elementary charges on a particle (-) \\
\hline $\mathscr{N}$ & Avogadro constant $\left(6.0221367 \cdot 10^{23} \mathrm{~mol}^{-1}\right)$ \\
\hline$N_{\text {jet }}$ & number of showerhead holes $(-)$ \\
\hline$P^{j e t}$ & gas pressure $\left(\right.$ dyne $/ \mathrm{cm}^{2}$ or $\left.\mathrm{g} \mathrm{cm}^{-1} \mathrm{~s}^{-2}\right)\left(1 \mathrm{~atm}=760\right.$ torr $\left.=1.01325 \cdot 10^{6} \mathrm{dyne} / \mathrm{cm}^{2}\right)$ \\
\hline$Q$ & volumetric gas flow rate $\left(\mathrm{cm}^{3} \mathrm{~s}^{-1}\right)$ \\
\hline$r$ & radial coordinate in axisymmetric geometry $(\mathrm{cm})$ \\
\hline$R$ & universal gas constant $\left(8.31451 \cdot 10^{7} \mathrm{~g} \mathrm{~cm}^{2} \mathrm{~s}^{-2} \mathrm{~K}^{-1} \mathrm{~mol}^{-1}\right)$ \\
\hline $\boldsymbol{R}_{\text {crit }}$ & starting radial position of a particle that deposits on the wafer's edge $(\mathrm{cm})$ \\
\hline$R_{W}$ & wafer (and showerhead) radius $(\mathrm{cm})$ \\
\hline$S$ & axial distance between wafer and showerhead $(\mathrm{cm})$ \\
\hline$t$ & time $(s)$ \\
\hline$T$ & gas temperature $(\mathrm{K})$ \\
\hline $\boldsymbol{U}$ & gas local velocity vector $(\mathrm{cm} / \mathrm{s})$ \\
\hline$U_{0}$ & magnitude of the mean axial (or face) velocity at showerhead exit $(\mathrm{cm} / \mathrm{s})$ \\
\hline$U_{\text {jet }}$ & local gas velocity in showerhead hole $(\mathrm{cm} / \mathrm{s})$ \\
\hline $\bar{U}_{\text {jet }}$ & mean gas velocity in showerhead hole $(\mathrm{cm} / \mathrm{s})$ \\
\hline
\end{tabular}




$\begin{array}{ll}\boldsymbol{V}_{\boldsymbol{p}} & \text { particle velocity vector }(\mathrm{cm} / \mathrm{s}) \\ V_{p}^{t} & \text { net particle axial drift velocity }(\mathrm{cm} / \mathrm{s}) \\ V_{E}^{t} & \text { particle electric axial drift velocity }(\mathrm{cm} / \mathrm{s}) \\ V_{G}^{t} & \text { particle gravitational axial drift velocity }(\mathrm{cm} / \mathrm{s}) \\ V_{T}^{t} & \text { particle thermophoretic axial drift velocity }(\mathrm{cm} / \mathrm{s}) \\ V_{p o} & \text { particle axial velocity at showerhead exit }(\mathrm{cm} / \mathrm{s}) \\ z & \text { axial coordinate in axisymmetric geometry }(\mathrm{cm})\end{array}$

Greek

$\alpha \quad$ empirical coefficient in slip-correction factor (-)

$\beta \quad$ empirical coefficient in slip-correction factor $(-)$

$\delta \quad$ dimensionless axial position at which external force resisting deposition and downward drag force are equal (-)
$\gamma \quad$ empirical coefficient in slip-correction factor (-)
$\phi \quad$ coefficient in gas mean free path definition (0.491)
$\eta \quad$ particle collection efficiency (-)
$\lambda \quad$ gas mean free path $(\mathrm{cm})=\mu /(\phi \rho \bar{c})$
$\mu \quad$ gas viscosity $\left(\mathrm{g} \mathrm{cm}^{-1} \mathrm{~s}^{-1}\right)$
$v \quad$ gas kinematic viscosity $\left(\mathrm{cm}^{2} \mathrm{~s}^{-1}\right)$
$\rho \quad$ gas density $\left(\mathrm{g} / \mathrm{cm}^{3}\right)=P M / R T$ (for ideal gas)
$\rho_{p} \quad$ particle density $\left(\mathrm{g} / \mathrm{cm}^{3}\right)$
$\tau \quad$ particle response time (s)
$\Lambda \quad$ particle volumetric source term $\left(\# \mathrm{~cm}^{-3} \mathrm{~s}^{-1}\right)$
$\Lambda_{h} \quad$ particle area source term $\left(\# \mathrm{~cm}^{-2} \mathrm{~s}^{-1}\right)$

\section{Dimensionless Parameters}

$C$

$C_{D}$

$\tilde{h}$

$\mathrm{Kn}$

$L / S$

$\mathrm{Pe}$

$\mathrm{Re}$

$\mathbf{R e}_{j e t}$

$\mathrm{Re}_{p}$

Sh

St

$\bar{U}_{\tilde{V}^{t}} / U_{o}$

Notation slip correction factor for particle drag .

particle drag coefficient

particle trap position $=h / S$

particle Knudsen number $=2 \lambda d_{p}$

ratio of showerhead thickness to inter-plate spacing

particle Peclet number $=S U_{o} / \mathcal{D}$

Reynolds number for parallel plate flow $=\rho U_{o} S / \mu$

Reynolds number for showerhead hole flow $=\rho \bar{U}_{j e t} d_{j e t} / \mu$

particle Reynolds number $=\rho d_{p}\left|\boldsymbol{V}_{p}-\boldsymbol{U}\right| / \mu$

particle Sherwood number $=\left.\frac{d \tilde{n}}{d \tilde{z}}\right|_{\tilde{z}=0}$

particle Stokes number $=\tau U_{o} / S$

ratio of showerhead hole mean velocity to face velocity

dimensionless net particle drift velocity $=V_{p}^{t} / U_{o}$ 


\section{Introduction}

A major cause of semiconductor yield degradation is contaminant particles that deposit on wafers while they reside in the many processing tools required to manufacture integrated circuits (Cooper, 1986; Bowling and Larrabee, 1989). Particle deposition on a wafer can occur during wafer handling (e.g., robot arm manipulations or door openings), process set up (e.g., establishing flows, pressures, or temperatures), or during the actual process step (e.g., chemical vapor deposition, plasma etch, or sputtering). The probability that a particle is a "killer defect" depends on both the properties of the particle and at which step the deposition occurred. Thus, it is of great interest to the industry to understand when, where, and how particles deposit on wafers during manufacturing. Donovan et al. (1993) reviewed the mechanisms of particle transport in process equipment and also provided qualitative experimental verification of these models. Bowling and Larrabee (1989) recognized the increasing number of processes being carried out at low pressure, and reported analytic and experimental results for the gravitational deposition of particles in a vacuum chamber. Periasamy et al. (1993) looked at low-pressure particle deposition mechanisms including sedimentation, convective-diffusion, thermophoresis, and other phoretic forces. Periasamy et al. (1993) also provided qualitative experimental verification of their models in a custom-built vacuum chamber.

Several studies have looked at fluid, mass, and heat transfer in geometries representative of process tools. For example, authors have used stagnation flow (Houtman et al., 1986), rotating disk flow (Evans and Grief, 1987a), or a combination of the two (Evans and Grief, 1988) to simulate chemical vapor deposition (CVD) process tools. The work of Houtman et al. (1986) is of particular relevance, as the stagnation-point flow geometry they investigated is closely related to the geometry of interest in this work. A major goal in all of these studies was to characterize the film deposition process; in particular, to identify conditions under which uniform deposition is obtained. Note that none of these studies addressed particle transport phenomena. Busnaina et al. (1993) have reported work on gas flow, heat transfer, and particle transport in a horizontal diffusion furnace; particle trajectories were calculated by solving the Lagrangian equation of motion including effects of temperature, drag, lift, gravity, and Brownian motion.

A large body of work has been developed in the area of particle deposition in infinite stagnation-point flows, approximating the case of a wafer exposed to a clean-room air flow. Axisymmetric flows have been used to simulate a free-standing wafer, while planar flows have been used to simulate a horizontal wafer placed on a bench. Based on a review of the pertinent heat and mass transfer experimental literature and using the heat/mass/particle transfer analogy, Liu and Ahn (1987) proposed that particle deposition under simultaneous convective diffusion and sedimentation can be approximated as the sum of the two independent deposition velocities. Experiments have shown that Liu and Ahn's approximate model works acceptably well in many cases (Otani et al., 1989; Pui et al., 1990; Schmidt et al., 1992; Bae et al., 1994). More detailed solutions for particle deposition rates in stagnation-point flows were later obtained using direct numerical simulations of the fluid, thermal, and particle transport equations (e.g., Stratmann et al., 1988; Peterson et al., 1989; Ye et al., 1991; Opiolka et al., 1994), or by approximations based on boundary-layer theory (e.g., Yuu and Jotaki, 1978; Dabros and van de Ven, 1983; Chari and Rajagopalan, 1985; Stratmann et al., 1988; Peterson et al., 1989; Ramarao and Tien, 1989; Cooper et al., 1989, 1990; Peters et al., 1989). Recently, Broday et al. (1997) have challenged the latter class of work in which infinite plate (viscous or potential) flows were assumed, and they have proposed a physically realistic analytical model for the stagnation flow over a finite flat obstacle. Several investigators (Yuu and Jotaki, 1978; Peters et al., 1989; Peterson et al., 1989; Ramarao and Tien, 1989; Peters and Cooper, 1991) have also considered enhanced deposition caused by particle inertial effects in stagnation-point flow. Collectively, these works provide a thorough description of particle deposition in infinite stagnation-point flow resulting from convection, diffusion, inertia, and external forces (including the gravitational, electrostatic, and thermophoretic forces). Experimental studies have typically reported good agreement between data and model predictions (Wu et al., 1989; Ye et al., 1991; Opiolka et al., 1994). Although the physical geometries of an isolated wafer in an infinite external flow and a wafer enclosed in a process tool seem very different, in both cases the flowfield very near the wafer can be approximated by a viscous, axisymmetric stagnation-point flow. 
This report investigates particle transport in an enclosed, parallel-plate reactor geometry characteristic of a wide range of single-wafer process tools. The axisymmetric geometry we consider consists of uniform flow exiting a showerhead separated by a small gap from a parallel wafer, as shown in Figure 1. The wafer typically rests on a susceptor, but for the present analysis the details of the susceptor are ignored. The showerhead consists of a material (usually a metal or ceramic) through which a large number of holes are drilled (see Figure 1a). As one major function of the showerhead is to evenly distribute the flow across its face, the holes are usually made very small in diameter and are very numerous (hundreds to thousands for an 8 inch wafer process tool). Ideally, a showerhead would produce a flow characterized by a mean axial (or face) velocity that does not vary in the radial direction; such flow uniformity is needed to accomplish uniform deposition or etching of the wafer surface. In practice, however, commercial showerheads are typically designed empirically to improve process parameters (such as uniformity); the resulting showerhead designs often create nonuniform flow fields which compensate for other system deficiencies - such as radial temperature or reactive species gradients. Various flow fields can be obtained by manipulation of showerhead hole sizes, numbers, and positions.

One common feature of showerhead design is that the area available to the flow is constricted inside the showerhead; consequently, the velocity of the gas inside the holes of the showerhead is much larger than the face velocity in the gap below. Particles originating upstream of the showerhead and suspended in the flow can be dramatically accelerated while passing through the showerhead, so that at the exit of the showerhead particle velocities much larger than the fluid face velocity are possible. Depending on conditions, particle acceleration by the showerhead can lead to inertia-enhanced particle deposition on the wafer below (Rader and Geller, 1998). Thus, a complete description of particle deposition on a wafer in a parallel-plate reactor must include a description of particle transport through the showerhead as well as an analysis of particle transport in the inter-plate region.

This report presents numerical models for assessing particle transport and deposition in a parallelplate geometry. No attempt is made here to analyze particle generation mechanisms; for the present discussion, particles are assumed to originate either: 1) upstream of the showerhead with a known concentration or 2) from a specified position between the plates with a fixed number or at a known generation rate. Because of the variety of physical mechanisms controlling particle deposition (inertia, diffusion, fluid drag, and external forces), several mathematical formulations are used to describe the problem. The basic phenomena controlling particle transport and the underlying general equations are reviewed in Chapter 2; note that the field of particle transport is quite broad so that for brevity discussion proceeds with an emphasis on conditions encountered in semiconductor process tools (i.e., sub-atmospheric pressures and submicron particles). Included in the discussion are expressions for the following particle forces: fluid drag, gravity, thermophoresis, and electric. The concepts of particle drift velocity and stopping distance are introduced, and issues of continuum vs. free molecular particle transport are outlined.

The determination of particle transport in a reactor must always begin with a determination of the fluid flow and temperature fields. In this work, particle concentrations are assumed to be low enough to allow a dilute approximation, for which the coupling between the fluid and particle phases is oneway. In this case, the fluid/thermal transport equations can be solved either analytically or numerically neglecting the particle phase; the resulting velocity and temperature fields are then used as input for the particle transport calculations. ${ }^{1}$ In all of the present work isothermal flow is assumed, although small temperature differences are allowed to drive particle thermophoresis. Descriptions of the techniques used to calculate fluid flow in the parallel plate geometry are given in Chapter 3 . In this work both analytic and numerical solutions of the flow field are presented.

\footnotetext{
1. The dilute mixture approximation is certainly valid for simulations of commercial semiconductor process tools, as the particle concentrations are typically controlled to very low levels.
} 
a)

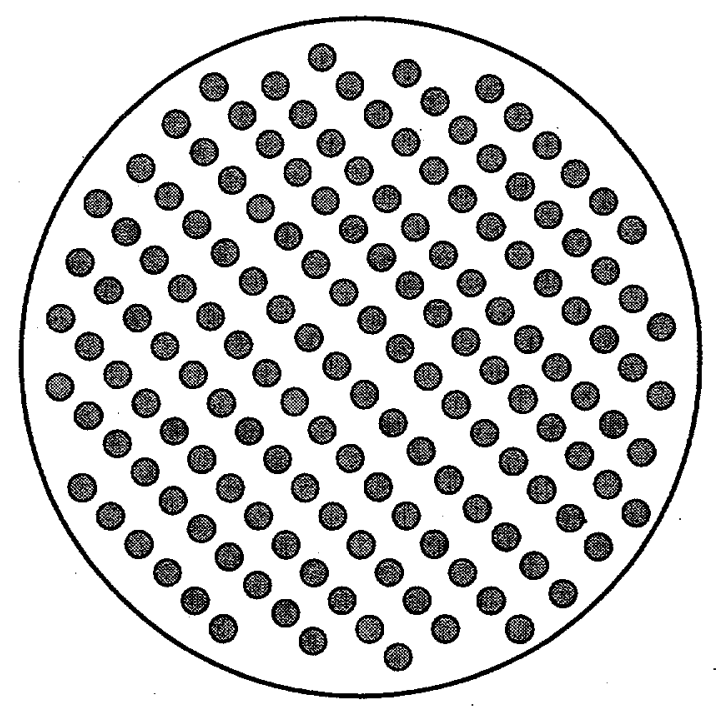

b)

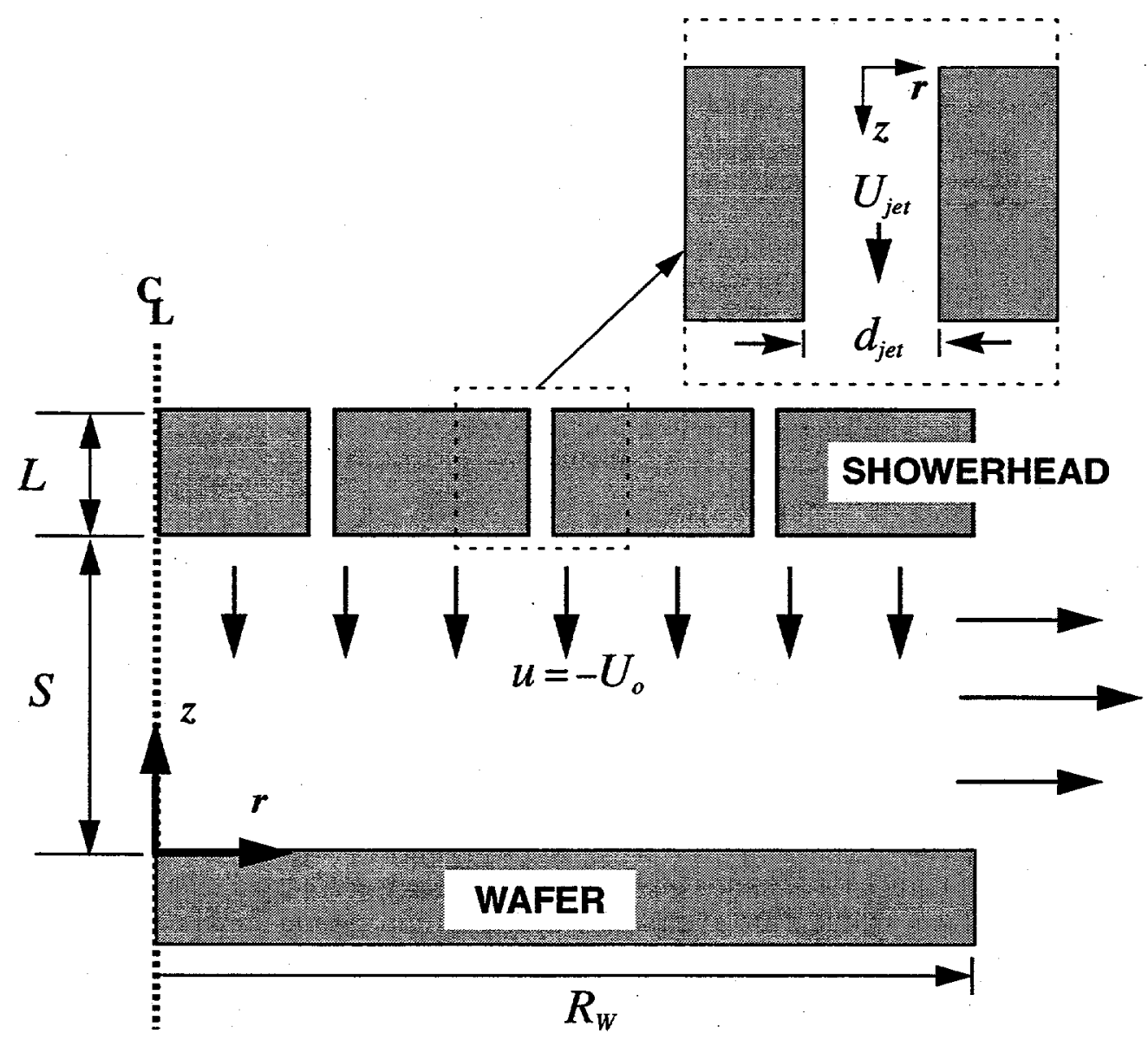

Figure 1. Parallel-plate Reactor Geometry. Schematic of the reactor geometry assumed in this work: a) top view of a showerhead and b) side view of a parallel-plate reactor. 
In order to provide a single parameter that can be used to compare particle deposition among many cases, Chapter 4 introduces a particle collection efficiency. The collection efficiency is defined as the fraction of particles that deposit on the wafer. Particles are presumed to either enter the reactor through the showerhead (uniformly spread between $r=0$ and $R_{W}$ ), or to originate in a plane parallel to the wafer. The latter case would correspond to particles being released from a plasma trap upon plasma extinction; in this case the particles are initially assumed to be uniformly spread radially between $r=0$ and $R_{W}$ at some distance $h$ from the wafer. Analytic expressions for collection efficiency are presented in Chapter 4 for the limiting case where external forces control deposition (i.e., neglecting particle diffusion and inertia).

Particle transport in the parallel-plate geometry is predicted using both a Lagrangian approach (where individual particle trajectories are calculated) and an Eulerian approach (where the particles are modeled as a continuum phase). The strength of the Eulerian formulation is in predicting particle transport resulting from the combination of applied external forces (including the fluid drag force) and the chaotic effect of particle Brownian motion (i.e., particle diffusion), although the current implementation cannot account for particle inertia. In particular, the Eulerian formulation cannot accommodate particle acceleration effects within the showerhead, and is therefore restricted to particle transport in the inter-plate region. The Eulerian formulation, presented in Chapter 5, yields an analytic description of particle deposition for the case where the flow field between the plates can be approximated analytically with a creeping-flow assumption and where the particles are assumed to originate from a planar trap located between the plates. An integral expression gives the particle collection efficiency as a function of four dimensionless parameters: $\operatorname{Re}, \tilde{h}, \tilde{V}_{p}^{t}$, and $\mathrm{Pe}$ (a fifth - the interception parameter $d_{p} / S$ - is neglected). The first parameter, the Reynolds number, completely specifies the flow field for the infinite parallel-plate geometry. The second parameter, the dimensionless trap height, $\tilde{h}=h / S$, specifies the position of the particle source term. The influence of external forces enters through the third parameter, the dimensionless particle drift velocity, $\tilde{V}_{p}^{t}=V_{p}^{t} / U_{o}$, which is defined as the $z$-component of the net drift velocity. The fourth parameter is the particle $\mathrm{Pe}$ clet number, $\mathrm{Pe}=S U_{o} / \mathcal{D}$, which is a measure of the relative importance of particle Brownian motion. In the free molecular limit the Peclet number is proportional to diameter squared, so that $\mathrm{Pe}^{1 / 2}$ can be thought of as a dimensionless particle size. The effects of these dimensionless parameters on collection efficiency are explored, and several examples are presented in dimensional terms. The need to properly account for diffusion-enhanced particle deposition becomes increasingly important as the semiconductor industry moves toward smaller feature sizes and becomes concerned with smallersized particles.

The strength of the Lagrangian formulation is in predicting particle transport resulting from the combination of applied external forces and particle inertia, although the current implementation cannot account for particle diffusion. It is the Lagrangian formulation, treated in Chapter 6, that can properly account for inertia-enhanced deposition resulting from particles which originate upstream of the showerhead and which are accelerated while passing through it. The problem is treated in two steps, in which particle and fluid transport are determined: 1) within a showerhead-hole, and 2) between the showerhead and wafer. For fluid and particle transport in the showerhead, approximate analytic expressions can be derived based on a few assumptions. The output of this first step is the particle velocity at the exit of the showerhead, as a function of showerhead geometry, flow rate, and gas and particle properties. The particle showerhead-exit velocity is next used as an initial condition required for particle transport between the plates. The output of the second step is a determination of whether the particle deposits on the wafer, as a function of showerhead-exit particle velocity, the plate separation, flow rate; and gas and particle properties. Note that the Lagrangian approach to the particle trajectory calculations is very similar to previous work in the classic inertial impactor geometry (Marple and Liu, 1974, 1975; Rader and Marple, 1985).

The final chapter summarizes the results of this study in terms of practical guidelines, i.e., recommendations of best-practices that are intended to help tool operators and designers reduce particle deposition. These guidelines are not intended to replace detailed calculations, but to provide the user with a general feel for inherently-clean practices. 


\section{Particle Transport}

This chapter reviews the theoretical models available to describe particle transport in typical semiconductor processing environments. Recent or classic references have been provided wherever possible for additional background. In all of the discussion that follows, particle concentrations are assumed to be low enough so that the influence of the particle on fluid transport can be neglected; particle-particle interactions are also neglected. Under this assumption, the fluid and thermal fields are calculated first (in the absence of particles), and then used as input for subsequent particle transport calculations. The theoretical underpinnings for both the Lagrangian approach (where individual particle trajectories are calculated) and the Eulerian approach (where the particle concentration field is modeled as a continuum) are presented. The strength of the Lagrangian formulation is in predicting particle transport resulting from external forces including particle inertia; but the current implementation can't describe the chaotic effect of particle Brownian motion (i.e., particle diffusion) on particle transport. On the other hand, the Eulerian formulation can describe particle transport resulting from applied forces and particle diffusion, but the current implementation can't account for particle inertia.

This chapter begins with a discussion of noncontinuum effects which play a key role in the transport of small particles at low pressure. Next follows a description of the Lagrangian particle transport equation, which basically describes how a particle responds when external forces are applied to it. Brief summaries of some of these forces (which are likely to be important inside a semiconductor process tool) are also included. The concept of particle response time is then introduced, which leads to a discussion of particle drift velocity. The chapter concludes with a description of the Eulerian particle transport equations, including a discussion of the particle diffusion coefficient.

\subsection{Noncontinuum Considerations}

In the following discussion, frequent mention will be made of the continuum and free molecular regimes. These terms are used here to distinguish between the two limiting cases characterizing the nature of the particle/gas interaction. ${ }^{1}$ In the continuum limit (large particles or high gas pressures), the gas surrounding the particle appears as a continuous fluid and traditional continuum fluid dynamics apply - such as the Navier-Stokes equations for fluid motion. In the free molecular limit (small particles or low gas pressures), however, the discrete nature of the gas becomes important and individual molecule/particle collisions must be considered. Discrimination between these two regimes is made by comparing the particle diameter to the gas mean free path (which is defined as the average distance a molecule travels between collisions with other gas molecules); a dimensionless parameter known as the Knudsen number is commonly used for these comparisons:

$$
\mathrm{Kn}=\frac{2 \lambda}{d_{p}}
$$

where:

$$
\begin{array}{ll}
\lambda & \text { gas mean free path }(\mathrm{cm})=\mu /(\phi \rho \bar{c}) \\
d_{p} & \text { particle diameter }(\mathrm{cm}) \\
\mu & \text { gas viscosity }\left(\mathrm{g} \mathrm{cm}^{-1} \mathrm{~s}^{-1}\right) \\
\rho & \text { gas density }\left(\mathrm{g} / \mathrm{cm}^{3}\right)=P M / R T \text { (for ideal gas) }
\end{array}
$$

\footnotetext{
1. The flowfield entraining the particle can also be either molecular or continuum in nature. In this case, discrimination between the two flow regimes is made by comparing some characteristic length associated with the reactor geometry to the gas mean free path. For example, a Knudsen number for the flowfield could be defined by replacing the particle diameter in Equation (2.1) with a characteristic reactor length scale. For small particles, it is frequently the case that the flow regime can be considered continuum while the fluid-particle interaction is characterized as free-molecular.
} 
$\bar{c} \quad$ mean thermal velocity of the gas molecules $(\mathrm{cm} / \mathrm{s})=(8 R T / \pi M)^{1 / 2}$

$R \quad$ universal gas constant $\left(8.3145 \cdot 10^{7} \mathrm{~g} \mathrm{~cm}^{2} \mathrm{~s}^{-2} \mathrm{~K}^{-1} \mathrm{~mol}^{-1}\right)$

$M \quad$ gas molecular weight ( $\mathrm{g} / \mathrm{mol})$

$P \quad$ gas pressure $\left(\right.$ dyne $/ \mathrm{cm}^{2}$ or $\left.\mathrm{g} \mathrm{cm}^{-1} \mathrm{~s}^{-2}\right)\left(1 \mathrm{~atm}=760\right.$ torr $\left.=1.01325 \cdot 10^{6} \mathrm{dyne} / \mathrm{cm}^{2}\right)$

$T$ gas temperature (K),

Also, $\phi$ is a dimensionless parameter that depends on the kinetic-theory model used to define the gas mean free path: in this work the value $\phi=0.491$ has been adopted (Allen and Raabe, 1982). Physical properties and the calculated mean free path are given for several gases in Table 1. At atmospheric pressures, the mean free path is typically less than $0.1 \mu \mathrm{m}$. Gas mean free path is inversely proportional to pressure at constant temperature; for example, the mean free path in air is $0.674 \mu \mathrm{m}$ at 76 torr, $6.74 \mu \mathrm{m}$ at 7.6 torr, and $67.4 \mu \mathrm{m}$ at 760 millitorr at $296 \mathrm{~K}$. Thus, for low-pressure applications, the Knudsen number for submicron particles can be large.

A large Knudsen number (say $>10$ ) corresponds to the free-molecular regime, while a small Knudsen number (say $<0.1$ ) corresponds to the continuum regime. Typically, verified theoretical expressions are available in the literature for the forces acting on particles in both the continuum and free-molecular limits. Unfortunately, theoretical force expressions are difficult to formulate in the transition regime that lies between the continuum and free-molecular regimes (particle size of the order of the mean free path, $K n=1$ ). Instead, interpolating or correlating functions are used which go to both the continuum and free-molecular expressions in the limit and match experimental data (if available) in between.

Table 1: Gas Properties. Density, viscosity, molecular weight, mean free path, and slip correction constants (for oil drops) for nine common gases at $T_{0}=296.15 \mathrm{~K}$ and $P_{o}=760$ torr (Rader, 1990).

\begin{tabular}{|l|c|c|c|c|c|c|c|}
\hline Gases & $\begin{array}{c}\mu_{o} \\
\left(\mathrm{~g} \cdot \mathrm{cm}^{-1} \mathrm{~s}^{-1}\right)\end{array}$ & $\begin{array}{c}M \\
\left(\mathrm{~g} \cdot \mathrm{mol}^{-1}\right)\end{array}$ & $\begin{array}{c}\rho_{o} \\
\left(\mathrm{~g} \cdot \mathrm{cm}^{-3}\right)\end{array}$ & $\begin{array}{c}\lambda_{o} \\
(\mu \mathrm{m})\end{array}$ & $\begin{array}{c}\alpha \\
(-)\end{array}$ & $\begin{array}{c}\beta \\
(-)\end{array}$ & $\begin{array}{c}\gamma \\
(-)\end{array}$ \\
\hline \hline Air & $183.47(-6)$ & 28.966 & $1.192(-3)$ & 0.0674 & 1.207 & 0.440 & 0.78 \\
\hline $\mathrm{Ar}$ & $224.80(-6)$ & 39.948 & $1.645(-3)$ & 0.0703 & 1.227 & 0.420 & - \\
\hline $\mathrm{He}$ & $197.11(-6)$ & 4.003 & $0.165(-3)$ & 0.1943 & 1.277 & 0.370 & 2.00 \\
\hline $\mathrm{H}_{2}$ & $88.61(-6)$ & 2.016 & $0.826(-3)$ & 0.1240 & 1.141 & 0.506 & - \\
\hline $\mathrm{CH}_{4}$ & $110.75(-6)$ & 16.043 & $0.661(-3)$ & 0.0545 & 1.154 & 0.493 & - \\
\hline $\mathrm{C}_{2} \mathrm{H}_{6}$ & $93.37(-6)$ & 30.069 & $1.251(-3)$ & 0.0333 & 1.254 & 0.393 & - \\
\hline$i-C_{4} \mathrm{H}_{10}$ & $75.06(-6)$ & 58.123 & $2.406(-3)$ & 0.0193 & 1.186 & 0.461 & - \\
\hline $\mathrm{N}_{2} \mathrm{O}$ & $147.88(-6)$ & 44.013 & $1.818(-3)$ & 0.0493 & 1.207 & 0.440 & - \\
\hline $\mathrm{CO}_{2}$ & $148.12(-6)$ & 44.010 & $1.823(-3)$ & 0.0438 & 1.150 & 0.497 & 0.92 \\
\hline
\end{tabular}




\subsection{Lagrangian Particle Equation of Motion}

In the Lagrangian approach to particle transport, particle Brownian motion is neglected and individual particle trajectories (position and velocity as a function of time) are determined by integrating the following system of ordinary differential vector equations:

$$
\begin{gathered}
\frac{d x_{p}}{d t}=V_{p} \\
m_{p} \frac{d V_{p}}{d t}=F_{D}+F_{G}+F_{T}+F_{E}+\Sigma F_{i}
\end{gathered}
$$

where $x_{p}$ is the particle position vector, $V_{p}$ is the particle velocity vector, $m_{p}$ is the particle mass, and $\boldsymbol{F}_{D}, \boldsymbol{F}_{G}, \boldsymbol{F}_{T} \boldsymbol{F}_{E}$, and $\boldsymbol{F}_{i}$ are the fluid-drag, gravitational, thermophoretic, electric, and any additional forces (diffusiophoresis, wall drag, etc.) acting on the particle. The forces explicitly listed in Equation (2.3) are those of greatest interest in the present analysis of parallel-plate tools; additional forces can be added linearly as needed. A review of these forces follows.

\subsection{Review of Particle Forces}

A brief review of the forces used in this report follows (for greater detail see Hinds, 1982; Donovan et al., 1993; or Rader and Geller, 1994). Because of the low pressures (typically less than 100 torr) and small particle sizes (diameters typically less than one micron) of interest in semiconductor processing, particles are usually sufficiently smaller than the gas mean free path, $\lambda$, so that the free-molecular limit of the various force expressions apply. The free-molecule assumption is well justified for particle Knudsen numbers ( $\mathrm{Kn}=2 \lambda d_{p}$ ) larger than ten, and is acceptable (accurate within about 20\%) for Knudsen numbers as small as two. For Knudsen numbers less than two, force expressions that extend into the transition or continuum regime should be used.

\subsubsection{Fluid-Particle Drag Force}

A particle moving at a different velocity than the surrounding gas will experience a gas resistance or fluid-drag force. A great deal of research has been devoted to describing the fluid-drag; only a brief review of this body of literature is reported here. For a rigid sphere of diameter $d_{p}$ moving at constant velocity $\boldsymbol{V}_{p}$ through a fluid with local velocity $U$ and viscosity $\mu$, the drag force is given by (e.g., see Friedlander, 1977, p.105):

$$
F_{D}=-\frac{3 \pi \mu d_{p}}{C(\mathrm{Kn})}\left(V_{p}-U\right) \cdot \mathrm{C}_{D}\left(\operatorname{Re}_{p}\right) \frac{\operatorname{Re}_{p}}{24}
$$

where $\mathrm{C}_{D}\left(\mathrm{Re}_{p}\right) \cdot \mathrm{Re}_{p} / 24$ is a non-Stokesian correction for gas inertial effects and $C(\mathrm{Kn})$ is a slip correction factor for noncontinuum effects. A fairly simple correlating equation for the non-Stokesian correction has been suggested by Turton and Levenspiel (1986):

$$
C_{D}\left(\operatorname{Re}_{p}\right) \frac{\operatorname{Re}_{p}}{24}=1+0.173 \operatorname{Re}_{p}^{0.657}+\frac{0.01721 \cdot \operatorname{Re}_{p}}{1+16,300 \cdot \operatorname{Re}_{p}^{-1.09}}
$$

where the particle Reynolds number is given as: 


$$
\operatorname{Re}_{p}=\frac{\rho d_{p}\left|V_{p}-U\right|}{\mu}
$$

and

$$
\left|V_{p}-\boldsymbol{U}\right|=\left[\left(u_{p}-u\right)^{2}+\left(v_{p}-v\right)^{2}+\left(w_{p}-w\right)^{2}\right]^{1 / 2}
$$

and $u, v$, and $w$ are the $x, y$, and $z$ components of velocity for the fluid (no subscript) and the particle (subscript $p$ ). This correlation applies for particle Reynolds number up to about 200,000 . Note that only moderate particle Reynolds numbers (say 10 at most) are expected for most semiconductor process applications, so that the far right terms in Equation (2.5) are small compared to unity. In fact, for submicron particles in typical processing environments, the slip-corrected Stokes drag law (Equation (2.4) with $\mathrm{C}_{D}\left(\operatorname{Re}_{p}\right) \cdot \mathrm{Re}_{p} / 24=1$ ) can generally be used with negligible error.

It has already been explained that, for small particles or at low gas pressures (large value of $\mathrm{Kn}$ ) the continuum approximation eventually breaks down and the molecular nature of the gas must be considered. Cunningham (1910) was the first to propose that a correction factor (called the Cunningham or slip correction factor), $C(\mathrm{Kn})$, be included in Equation (2.4) to account for noncontinuum effects. The functional form first suggested by Knudsen and Weber (1911) is in common use:

$$
C(\mathrm{Kn})=1+\mathrm{Kn}\left(\alpha+\beta \cdot \exp \left(-\frac{\gamma}{\mathrm{Kn}}\right)\right)
$$

where $\alpha, \beta$, and $\gamma$ are parameters that depend on the nature of the gas-particle interaction at the particle surface, and so are affected by both gas composition and particle surface roughness. For example, Ishida (1923) used a Millikan apparatus to determine the coefficient $\alpha$ for oil-drops in nine common gases. His results were recently re-evaluated by Rader (1990) using modern, more accurate values for the electric charge and gas properties; the corrected values for $\alpha$ are given in Table 1.

Based on theoretical considerations (Millikan, 1923) and experimental data (Eglin, 1923), Rader (1990) recommends that the expression $\alpha+\beta=1.647$ can be used to accurately calculate $\beta$ for most gas and particle-surface combinations; $\beta$ values calculated by this formula using Ishida's measured values for $\alpha$ are given in Table 1. The choice of the third constant, $\gamma$, is more difficult as there are no theoretical techniques for estimating it, and as complete data for empirically determining it are limited. Rader (1990) fit the available data for air, carbon dioxide, and helium and found $\gamma$ values of $0.78,0.92$, and 2.0 , respectively. For other gases, Rader (1990) suggests $\gamma=0.85$. Note that the correlation for the slip correction factor given by Equation (2.8) is not very sensitive to the value of $\gamma$ used. In fact, only small errors typically result if the slip factor is calculated using the fitted constants for oil-droplets in air ( $\alpha$ $=1.207, \beta=0.440$, and $\gamma=0.78$ ) for different gases or for particles of different surface roughness. A plot of the $C(\mathrm{Kn})$ against $\mathrm{Kn}$ is given in Figure 2 using these constants. Note that $C(\mathrm{Kn})$ approaches unity in the continuum limit, and approaches $(\alpha+\beta) \mathrm{Kn}$ in the free molecule limit.

\section{Fluid-Drag Force: Assumptions and Practical Considerations}

All of the above formulae assume solid, homogeneous, spherical particles, while real particles may be far from spherical in shape (flakes, rods, deformed droplets, etc.) and may be porous or inhomogeneous in composition. These variations from ideality can result in particle rotation, modifications to the drag law, etc. Two methods are commonly used to account for nonsphericity: the use of an equivalent diameter or the correction of the drag law with a dynamic shape factor. Hinds (1982, p.47) and Fuchs (1964, p.37) provide discussion of both of these approaches. Techniques are becoming available for more accurately modeling the transport of certain classes of nonspherical particles (including flakes, rods, chains of spheres, etc.), but these greatly increase the complexity of the problem. 


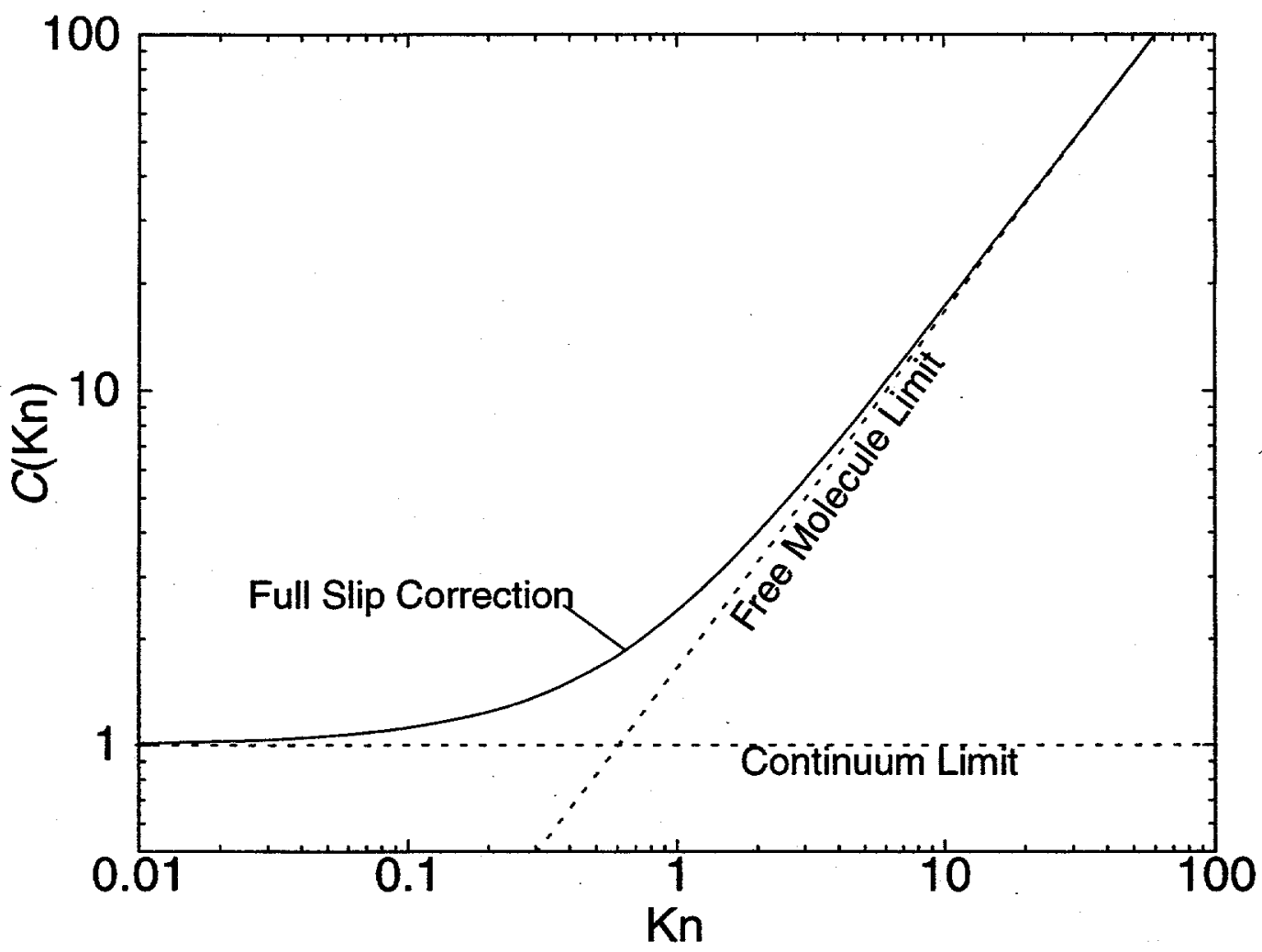

Figure 2. Slip Correction Factor. Dependence of the slip correction factor on particle Knudsen number (solid curve). The free molecular and continuum limits for the slip correction factor are also shown (dotted curves).

In adopting Equation (2.4), resistance terms resulting from fluid inertia (e.g., the virtual mass and Basset history integral terms) have been neglected, which has been shown (Fuchs, 1964; Clift et al., 1978) to be a reasonable approximation for aerosol particles (where the particle density is much larger than the fluid density) at particle Reynolds numbers not exceeding a few hundred. The uncertainty in Equation (2.4) becomes greater at higher Reynolds numbers, but high Reynolds numbers are not expected in semiconductor applications.

Equation (2.4) strictly applies to the uniform (non-accelerating), straight-line motion of a sphere in a quiescent fluid. In the present application, however, the drag on an accelerating particle in a nonuniform flow field is needed. Fuchs (1964, Chapter 3) and Clift et al. (1978, Chapter 11) review the issues related to the non-uniform rectilinear motion of aerosol particles. Although it will introduce some inaccuracy, the instantaneous drag acting on an accelerating particle can be estimated with the above constant-velocity drag expression with the fluid and particle velocities taken as their local, instantaneous velocities.

Strictly speaking, the combination of the slip $C(\mathrm{Kn})$ and non-Stokesian $\left(\mathrm{C}_{D}\left(\mathrm{Re}_{p}\right) \cdot \mathrm{Re}_{p} / 24\right)$ corrections in Equation (2.4) as a product is on flimsy grounds. Henderson (1976), for example, presents a correlation that adds the corrections. ${ }^{2}$ Unfortunately, there is little or no data available for Re 1 where slip is appreciable, so the proper formulation can't be decided. For typical semiconductor process environments, however, particle Reymolds numbers are likely to be low so that the non-Stokesian correction is near one and Equation (2.4) is likely to be quite satisfactory. In practice, non-Stokesian effects can be neglected for particle Reynolds numbers less than $\sim 0.3$. Thus, in the rest of this work, the slip-corrected Stokes drag law (Equation (2.4) with $\mathrm{C}_{D}\left(\operatorname{Re}_{p}\right) \cdot \mathrm{Re}_{p} / 24=1$ ) will be used.

\footnotetext{
2. $C$ is removed from Equation (2.4) and the expression $1 / C$ replaces " 1 " as the first term on the right hand side of Equation (2.5)
} 


\section{Continuum Regime Limit}

In the continuum limit (large particles and/or near-atmospheric process pressures) and assuming non-Stokesian effects can be neglected, Equation (2.4) for the gas resistance reduces to Stokes law (e.g., see Hinds, 1982, p.41):

$$
F_{D, \text { continuum }}=-3 \pi \mu d_{p}\left(V_{p}-U\right)
$$

The continuum regime drag force is seen to be directly proportional to particle size and to the velocity difference between the particle and the gas; it is independent of process pressure (the dependence of fluid viscosity on pressure is very weak) and depends on temperature only through the temperature dependence of viscosity.

\section{Free Molecule Regime Limit}

In the free molecular limit (small particles and/or low process pressures) and assuming non-Stokesian effects can be neglected, Equation (2.4) for the gas resistance reduces to the following free molecular result (similar to that originally derived by Epstein, 1924) in the limit of large $\mathrm{Kn}$ :

$$
F_{D, \text { molecular }}=-\frac{3 \pi \phi}{2(\alpha+\beta)} \rho \bar{c} d{ }_{p}^{2}\left(V_{p}-U\right)
$$

As in the continuum limit, free-molecular drag force is directly proportional to the gas-particle velocity difference. Unlike the continuum result, however, free molecular drag shows a much stronger (squared) dependence on particle diameter. Another difference is that free-molecular drag is directly proportional to process pressure (through the fluid density term). Temperature dependencies arise implicitly through the fluid density and the mean gas velocity.

\subsubsection{Gravitational Force}

The gravitational force acting on a spherical particle is given by Hinds (p.42, 1982):

$$
F_{G}=\frac{\pi}{6} d_{p}^{3}\left(\rho_{p}-\rho\right) g
$$

where $\rho_{p}$ and $\rho$ are the particle and gas density, respectively, and $\boldsymbol{g}$ is the gravitational acceleration vector. The gas density is typically much smaller than the particle density, and can be neglected in Equation (2.11). The gravitational force is independent of the gas mean free path.

\subsubsection{Thermophoretic Force}

Particles suspended in a gas with a temperature gradient will experience a force in the direction of decreasing temperature. The resulting motion of particles away from hot regions and towards cold regions is called thermophoresis. Thermophoresis results in the preferential deposition of particles on a cold wall, and explains the appearance of a particle free zone near a hot wall. An accepted formulation for the thermophoretic force on a spherical particle was developed by Talbot et al. (1980) in a review of thermophoresis:

$$
F_{T}=-3 \pi \mu d_{p} v K_{T} \cdot \frac{\nabla T}{T}
$$

where 


$$
K_{T}=\frac{2 C_{s}\left(\frac{k_{g}}{k_{p}}+C_{t} K n\right)}{\left(1+3 C_{m} K n\right)\left(1+2 \cdot \frac{k_{g}}{k_{p}}+2 C_{t} K n\right)}
$$

and where $\nabla T$ is the temperature gradient in the gas, $T$ is the mean gas temperature about the particle, $v=\mu / \rho, k_{g}$ and $k_{p}$ are the gas and particle thermal conductivities, ${ }^{3}$ and $C_{t}, C_{s}, C_{m}$ are the thermal creep coefficient, temperature jump coefficient, and velocity jump coefficient, respectively $\left(C_{s}=1.147\right.$, $C_{t}=2.20$, and $C_{m}=1.146$ are recommended by Batchelor and Shen, 1985). Talbot et al. (1980) compared their correlation with other experimenters data over a wide range of Knudsen numbers, and found it agrees with available experimental data to within $20 \%$.

\section{Continuum Regime Limit}

In the continuum limit of small $\mathrm{Kn}$ the thermophoretic force approaches:

$$
F_{T, \text { continuum }}=-6 \pi \mu^{2} d_{p} \cdot \frac{C_{s} k_{g}}{k_{p}+2 k_{g}} \cdot \frac{\nabla T}{\rho T}=-6 \pi \mu^{2} d_{p} \cdot \frac{C_{s} k_{g}}{k_{p}+2 k_{g}} \cdot \frac{R \nabla T}{P M}
$$

which is proportional to particle diameter and the temperature gradient. Note that the final equality applies for an ideal gas, for which the continuum-limit force is inversely proportional to pressure.

\section{Free Molecule Regime Limit}

In this limit Equation (2.12) approaches the limit first derived by Waldmann and Schmitt (1966):

$$
F_{T, \text { molecular }}=-\frac{\pi}{2} \phi \mu \bar{c} d_{p}^{2} \cdot \frac{\nabla T}{T}
$$

Note that in the free-molecular limit the thermophoretic force is proportional to diameter squared and is independent of the gas pressure and the particle thermal conductivity. ${ }^{4}$

\subsubsection{Electrostatic Force}

A charged particle suspended in a region with an electric field $E$ will experience a force (Hinds, 1982, p. 286):

$$
F_{E}=n_{p} e E=q E
$$

where $n_{p}$ is the number of elementary charge units $e\left(4.803 \times 10^{-10}\right.$ esu in cgs) on the particle giving a total charge $q$. This equation is deceptively simple, in that the determination of either the charge on the particle or the surrounding electric field can be exceedingly challenging (experimentally or theoretically). In a plasma tool, for example, $n_{p}$ and $E$ will change with time and position and may not be independent.

\footnotetext{
3. For polyatomic gases, Talbot et al. recommend the use of the 'translational' thermal conductivity, which is given by simple kinetic theory as $k_{g}=(15 / 4) \mu R / M$.

4. The gas conductivity does play a role, but has been eliminated from Equation (2.15) by the expression in footnote 4 .
} 


\subsection{Inertial Effects}

Using the particle fluid-drag force relationship (Equation (2.4) with $\mathrm{C}_{D}\left(\operatorname{Re}_{p}\right) \cdot \mathrm{Re}_{p} / 24=1$ ), the particle force balance of Equation (2.3) can be rewritten:

$$
\tau \frac{d \boldsymbol{V}_{p}}{d t}=\boldsymbol{U}-\boldsymbol{V}_{p}+\frac{C(\mathrm{Kn})}{3 \pi \mu d_{p}}\left(\boldsymbol{F}_{G}+\boldsymbol{F}_{T}+\boldsymbol{F}_{E}+\Sigma \boldsymbol{F}_{i}\right)=U-\boldsymbol{V}_{p}+\boldsymbol{V}_{G}^{t}+\boldsymbol{V}_{T}^{t}+\boldsymbol{V}_{E}^{t}+\Sigma \boldsymbol{V}_{i}^{t}
$$

where we have introduced a particle response or relaxation time

$$
\tau=\frac{\rho_{p} d_{p}^{2} C(\mathrm{Kn})}{18 \mu}
$$

and the particle drift velocity $V_{i}^{t}$ which is discussed below in Section 2.5. Particles characterized by small response times respond rapidly to changes in the flow or in the applied forces, while particles with large response times respond slowly to such changes. For large response times, particle transport is dominated by particle inertia.

\subsubsection{Nondimensionalization}

It is often convenient to nondimensionalize Equations (2.2) and (2.17). For this purpose, a characteristic length and velocity must be specified. For example, for the parallel plate geometry (see Figure 1), we could chose the inter-plate separation $S$ as the characteristic length, and the magnitude of the mean face velocity $U_{o}$ as the characteristic velocity, for which Equations (2.2) and (2.3) become:

$$
\begin{gathered}
\frac{d \tilde{\boldsymbol{x}}_{p}}{d \tilde{t}}=\tilde{\boldsymbol{V}}_{p} \\
\mathrm{St} \frac{d \tilde{\boldsymbol{V}}_{p}}{d \tilde{t}}=\tilde{\boldsymbol{U}}-\tilde{\boldsymbol{V}}_{p}+\left(\tilde{\boldsymbol{V}}_{G}^{t}+\tilde{\boldsymbol{V}}_{T}^{t}+\tilde{\boldsymbol{V}}_{E}^{t}+\Sigma \tilde{\boldsymbol{V}}_{i}^{t}\right)
\end{gathered}
$$

where $\tilde{x}_{p}=x_{p} / S, \tilde{t}=t U_{o} / S, \tilde{V}_{p}=V_{p} / U_{o}, \tilde{U}=U / U_{o}$, and $\tilde{V}_{i}^{t}=V_{i}^{t} / U_{o}$ where $V_{i}^{t}$ is the particle drift velocity described below. We have also introduced the particle Stokes number, St, which is dimensionless number defined as:

$$
\mathrm{St}=\frac{\tau U_{o}}{S}=\frac{\rho_{p} d_{p}^{2} C(\mathrm{Kn}) U_{o}}{18 \mu S}
$$

where $\tau$ is the particle relaxation time. The Stokes number is a convenient measure of the importance of particle inertia in a specific reactor; for small St inertial effects can be neglected, while for large $\mathrm{St}$ inertial effects must be considered. Physically, the Stokes number can be interpreted as the ratio of the particle stopping distance ${ }^{5}$ to the characteristic length of system. The continuum and free molecule limits of St are:

\footnotetext{
5. The particle stopping distance, $\tau U_{0}$, is defined as the distance a particle would travel before stopping if injected into a quiescent fluid at an initial velocity of $U_{0}$.
} 
Continuum Regime Limit:

$$
\mathrm{St}_{\text {continuum }}=\frac{\rho_{p} d_{p}^{2} U_{o}}{18 \mu S}
$$

Free Molecule Regime Limit:

$$
\mathrm{St}_{\text {molecular }}=\frac{\alpha+\beta}{9 \phi} \cdot \frac{\rho_{p} d_{p} U_{o}}{\rho \bar{c} S}=0.373 \cdot \frac{\rho_{p} d_{p} U_{o}}{P S} \cdot\left(\frac{\pi R T}{8 M}\right)^{1 / 2}
$$

\subsection{Drift Velocity}

The manipulation of Equation (2.3) into Equation (2.17) resulted in the introduction of the particle drift velocity, $V_{i}^{t}$, which can be shown to be the particle velocity at which the $i^{t h}$ force (neglecting all others) acting on the particle exactly balances the fluid drag force retarding the motion. At this balance point, the particle acceleration vanishes and the particle would move at a steady (or terminal) velocity which we shall refer to as the drift velocity. The time required for the particle to reach the drift velocity is short for particles characterized by small particle response times (small Stokes numbers). For small Stokes numbers, particle inertia can be neglected and the acceleration term on the left hand side of Equation (2.17) can be dropped, so that particle velocity can be expressed as:

$$
V_{p}=U+V_{p}^{t}=U+V_{G}^{t}+V_{T}^{t}+V_{E}^{t}+\Sigma V_{i}^{t}
$$

where the net particle drift velocity is obtained by summing over all external forces, i.e.:

$$
\boldsymbol{V}_{p}^{t}=V_{G}^{t}+V_{T}^{t}+V_{E}^{t}+\Sigma V_{i}^{t}=\frac{C(K n)}{3 \pi \mu d_{p}}\left(F_{G}+F_{T}+F_{E}+\Sigma F_{i}\right)
$$

Equation (2.24) and Equation (2.25) show that, neglecting inertia, the particle will move with the fluid velocity plus the vector sum of the individual drift velocities from all of the applied external forces. In the absence of any external forces (and having neglected particle diffusion), a noninertial particle will exactly follow flow streamlines. For isothermal flow between horizontal, parallel plates, all the $V_{i}^{t}$ are constant and are directed normal to the plates. Expressions are given below for the drift velocities for each of the forces listed in Section 2.3.

\subsubsection{Gravitational Drift Velocity}

The drift (or settling) velocity for a spherical particle settling under gravity can be found from Equations (2.4) and (2.11) with buoyancy and non-Stokesian effects neglected:

$$
V_{G}^{t}=\frac{\rho_{p} d_{p}^{2} g C(\mathrm{Kn})}{18 \mu}
$$

Although the form of the gravitational force is the same in both the continuum and free molecule regime limits, expressions for the two drift velocity limits differ due to the drag law contribution: 


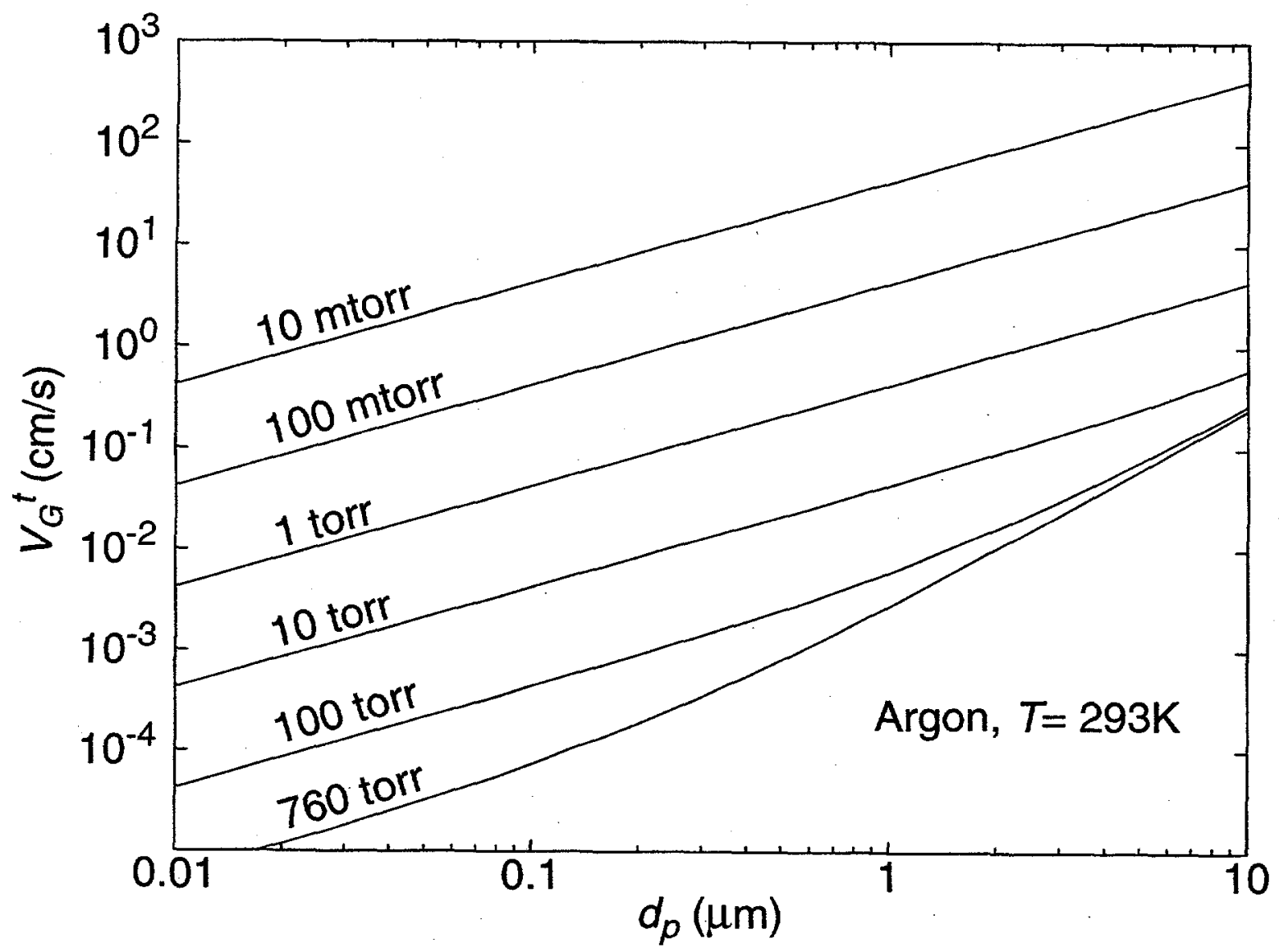

Figure 3. Gravitational Drift Velocity. Dependence of the gravitational drift (or settling) velocity on particle diameter for six process pressures (argon at $293 \mathrm{~K}$, particle density $\rho_{p}=1 \mathrm{~g} / \mathrm{cm}^{3}$ ).

Continuum Regime Limit:

$$
V_{G, \text { continuum }}^{t}=\frac{\rho_{p} d_{p}^{2} g}{18 \mu}
$$

Free Molecule Regime Limit:

$$
V_{G, \text { molecular }}^{t}=\frac{\alpha+\beta}{9 \phi} \cdot \frac{\rho_{p} d_{p} g}{\rho \bar{c}}=0.373 \cdot \frac{\rho_{p} d_{p} g}{P} \cdot\left(\frac{\pi R T}{8 M}\right)^{1 / 2}
$$

Note that the settling speed of a particle in the free molecule limit varies directly as particle diameter and inversely with pressure, while in the continuum limit the settling speed varies as diameter squared and is independent of pressure.

\section{Example}

The gravitational drift velocity for a unit density spherical particle as a function of particle size is shown in Figure 3 for six different process pressures in argon at $293 \mathrm{~K}$. For pressures below 100 torr and particle diameters below $1 \mu \mathrm{m}$, note that the lines are parallel and straight with a slope of one, as predicted by the free molecule regime limit. Thus, for most of the pressures and particle sizes of interest in semiconductor processing, the free molecule regime limit - Equation (2.28) - can be used to predict particle settling rates, greatly simplifying calculations. 


\subsubsection{Thermophoretic Drift Velocity}

The thermophoretic drift velocity resulting from the balance of thermophoretic and drag forces (neglecting non-Stokesian effects) alone can be found by equating Equations (2.4) and (2.12):

$$
V_{T}^{t}=-K_{T} C(K n) \frac{\mu \nabla T}{\rho T}=-K_{T} C(K n) \frac{\mu R \nabla T}{P M}
$$

Interestingly, the drift velocity given by Equation (2.29) depends on particle diameter only implicitly through the slip correction factor and $K_{T}$; specifically, in both the large and small particle limits the thermophoretic velocity becomes independent of particle size.

Continuum Regime Limit

$$
V_{T, \text { continuum }}^{t}=-\frac{2 C_{s} k_{g}}{k_{p}+2 k_{g}} \frac{\mu \nabla T}{\rho T}=-\frac{2 C_{s} k_{g}}{k_{p}+2 k_{g}} \frac{\mu R \nabla T}{P M}
$$

The continuum limit for thermophoretic drift velocity does not depend on particle size.

\section{Free Molecule Regime Limit}

In the free molecular limit, the thermophoretic drift velocity reduces to:

$$
V_{T, \text { molecular }}^{t}=-\frac{C_{s}(\alpha+\beta)}{3 C_{m}} \frac{\mu \nabla T}{\rho T}=-\frac{C_{s}(\alpha+\beta)}{3 C_{m}} \frac{\mu R \nabla T}{P M}=-0.549 \frac{\mu R \nabla T}{P M}
$$

which is the same result as given by Waldmann and Schmitt (1966). The free molecule limit is independent of particle size and gas/particle thermal conductivities. The numerical constant 0.549 in the final equality is very general as the sum $\alpha+\beta$ is nearly constant for most gases and particle surfaces.

Example

The thermophoretic drift velocity for a spherical particle as a function of particle size and pressure is shown in Figure 4 for an assumed temperature gradient of $\nabla T=1 \mathrm{~K} / \mathrm{cm}$. For the calculations, the ratio of gas to particle thermal conductivities was taken as $k_{g} / k_{p}=0.001$, representative of a metal particle suspended in argon (note that in the free-molecular regime the thermophoretic drift velocity is independent of $k_{g} / k_{p}$ ). Even for this small temperature gradient, the drift velocity can become large at low pressures. For pressures below 100 torr and particle diameters below $1 \mu \mathrm{m}$, note that the drift velocity becomes independent of particle diameter and inversely proportional to process pressure, as predicted for the free molecule regime limit. Thus, for must of the pressures and particle sizes of interest in semiconductor processing, the free molecule regime limit - Equation (2.31) - can be used to predict particle thermophoretic drift rates.

\subsubsection{Electric Drift Velocity}

The drift velocity for a spherical particle moving under an applied electric field is given by:

$$
V_{E}^{t}=\frac{C(K n)}{3 \pi \mu d_{p}} \cdot q E
$$

Although the electrical force is independent of process conditions such as temperature and pressure, these quantities enter the expression for drift velocity through the drag-law contribution. 


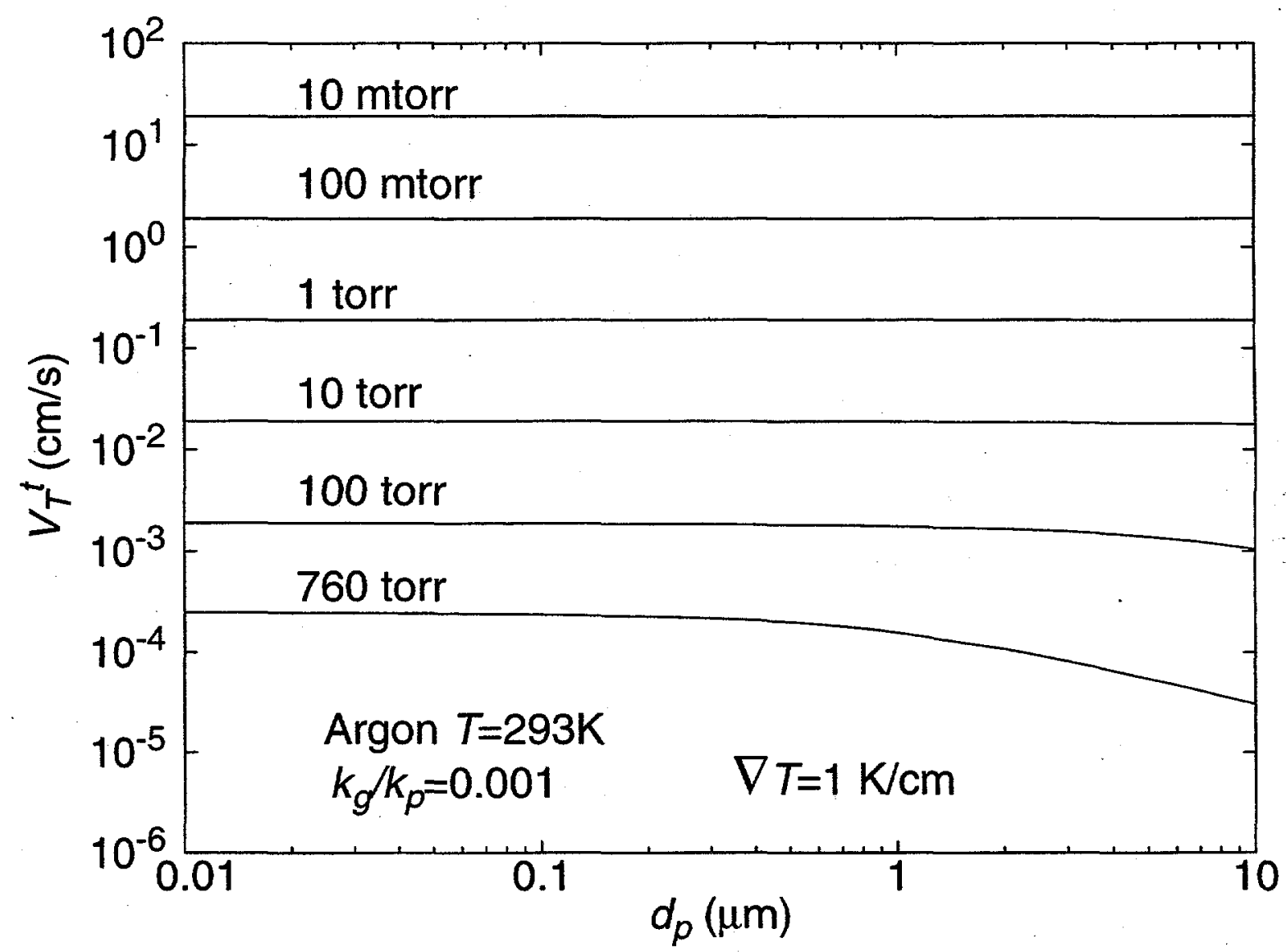

Figure 4. Thermophoretic Drift Velocity. Dependence of the thermophoretic drift velocity on particle diameter for six process pressures (argon at $293 \mathrm{~K}, k_{g} / k_{p}=0.001, \nabla T=1 \mathrm{~K} / \mathrm{cm}$ ).

\subsection{Eulerian Formulation}

While the Lagrangian (particle tracking) method predicts particle transport by considering single particle motion, the Eulerian formulation predicts particle transport by viewing the particle concentration field as a continuum. In this case, the solution of the particle transport problem becomes very much like that posed by the flow field, i.e., there is one continuous equation for particle mass (concentration) conservation and one continuous equation for particle momentum (velocity) conservation for each particle size. Particle transport by diffusion (Brownian motion) is naturally included in this formulation. A great simplification is obtained if particle inertia is neglected, i.e., it is assumed that the particle instantaneously reaches the drift velocity where drag and imposed forces are in balance. ${ }^{6}$ In this case, the particle momentum equation is no longer needed, and only the particle continuity equation for particle concentration $n$ (particles $/ \mathrm{cm}^{3}$ ) remains (Fuchs, 1964, p. 190; Peters et al., 1989):

$$
\begin{array}{llcc}
\text { NET CONVECTION } & \text { DIFFUSION } & \text { DRIFT } & \text { SOURCE } \\
\frac{\partial n}{\partial t}+ & \boldsymbol{U} \cdot \nabla n=\nabla \cdot \mathcal{D} \nabla n-\nabla \cdot\left(V_{p}^{t} n\right)+\Lambda
\end{array}
$$

\footnotetext{
6. The requirement that the characteristic time for particle diffusion is much longer than the particle characteristic time, $t \gg>$, is also essential to the development of the basic equations of particle transport by Brownian motion (see Fuchs, 1964, Section 35).
} 
where $\mathcal{D}\left(\mathrm{cm}^{2} / \mathrm{sec}\right)$ is the Stokes-Einstein particle diffusion coefficient (discussed below) and $\Lambda\left(\# / \mathrm{cm}^{3} /\right.$ sec) is a particle source/sink term to account for particle generation/consumption. The net drift velocity, $V_{p}^{t}$, appearing in Equation (2.33) is the same one discussed previously. Although we will not make further use of the fact, it is interesting to note that Equation (2.33) is applicable to either the Brownian motion of an individual particle or to the diffusion of a particle cloud taken from the continuum point of view (Fuchs, 1964, p. 191). For a single particle, $n$ is interpreted as the probability of finding a particle at position $(x, y, z)$ at time $t$ given that the particle was initially located at position $\left(x_{o}, y_{o}, z_{o}\right)$ at time $t_{o}$. Thus, although semiconductor applications are likely characterized by very low particle concentration levels, the continuum approach can still be applied if we continue to associate the particle concentration with a probability distribution (for example, we may find particle concentrations less than $1 \mathrm{~cm}^{-3}$, which is acceptable from a probabilistic point of view). For boundary conditions, it is assumed that particles which contact the wall stick and are thus instantly removed from the gas, so that the concentration $n$ equals zero at all walls.

Only one-way coupling between the fluid flow and particle concentration fields is used in this work; i.e., the flow field is coupled to particle transport through the velocity field $U$ which appears in Equation (2.33), while the influence of the particle phase upon the flow is neglected. In practice, the flow field is calculated first (in the absence of a particle phase) and the resulting velocity field is supplied to Equation (2.33) as a known solution.

\subsubsection{Particle Diffusion Coefficient}

A more complete discussion of the Stokes-Einstein particle diffusion and its derivation is available in any aerosol text (e.g., Fuchs, 1964, Chapter 5). The diffusion coefficient for a spherical particle is:

$$
\mathcal{D}=\frac{k T C(\mathrm{Kn})}{3 \pi \mu d_{p}}
$$

where $C(\mathrm{Kn})$ is defined by Equation (2.8). The validity of Equation (2.34) rests on several assumptions, such as that the particles move independently of one another and that the movements of a particle in consecutive time intervals are independent (Fuchs, 1964). The latter assumption is met only if the condition $t>>\tau$ holds true; in other words, the expression for the diffusion coefficient given in Equation (2.34) is only valid for observation times much longer than the particle response time.

\section{Continuum Regime Limit}

The continuum regime limit for the diffusion coefficient is:

$$
\mathcal{D}_{\text {continuum }}=\frac{k T}{3 \pi \mu d_{p}}
$$

which is inversely proportional to particle diameter and independent of pressure.

Free Molecule Regime Limit

In the free molecular limit, the diffusion coefficient reduces to:

$$
\mathcal{D}_{\text {molecular }}=\frac{(\alpha+\beta)}{3 \phi}\left(\frac{R T}{2 \pi M}\right)^{\frac{1}{2}} \frac{k T}{P d_{p}^{2}}
$$

which is inversely proportional to particle diameter squared and pressure. 


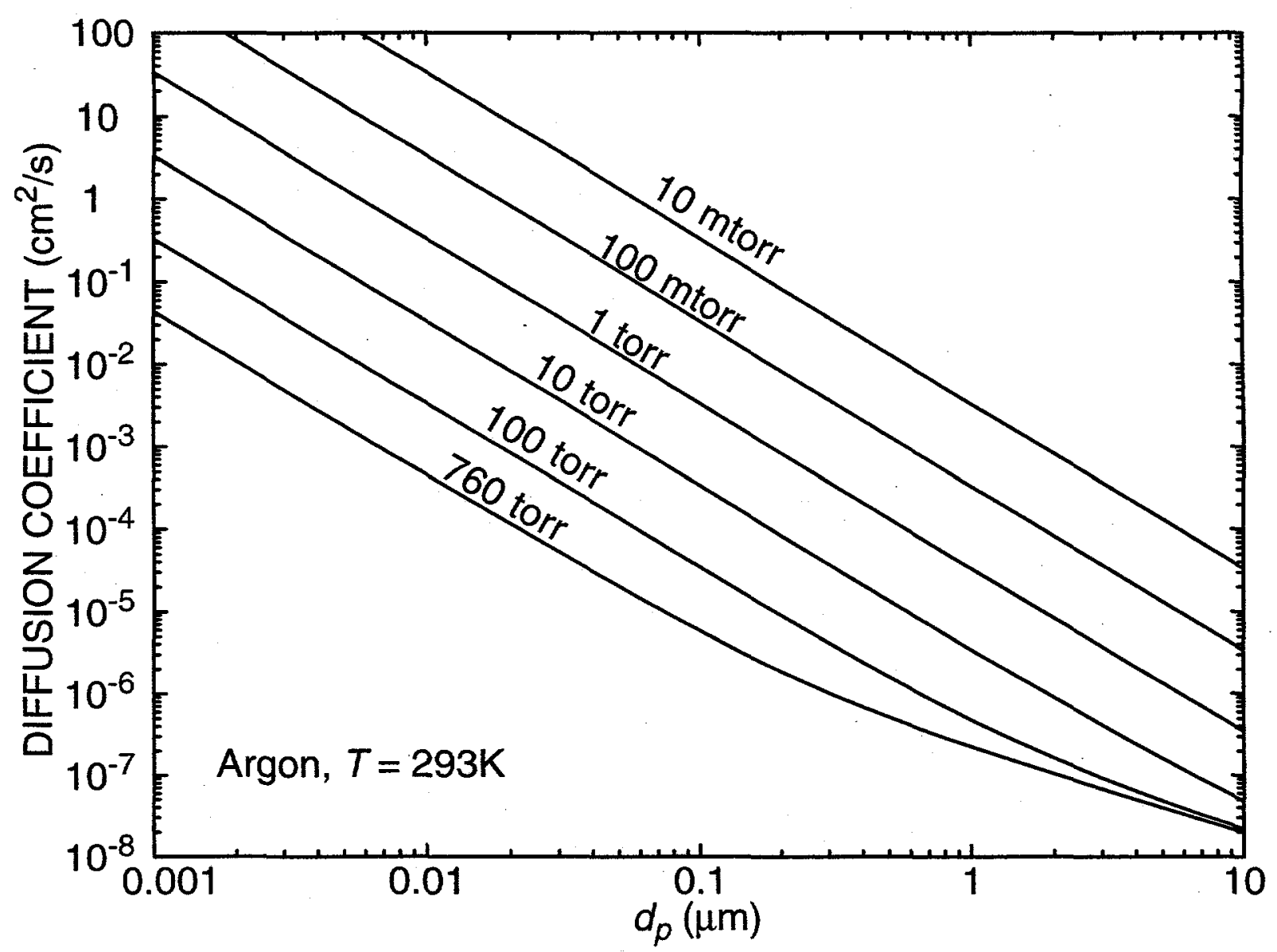

Figure 5. Diffusion Coefficient. Dependence of the particle diffusion coefficient on particle diameter for six process pressures in argon at $293 \mathrm{~K}$.

\section{Example}

The particle diffusion coefficient for a spherical particle as a function of particle size and pressure is shown in Figure 5 for a temperature of 293K. For pressures below 100 torr and particle diameters below $1 \mu \mathrm{m}$, note that the lines are parallel and straight with a slope of negative two, as predicted for the free molecule regime limit. Thus, for must of the pressures and particle sizes of interest in semiconductor processing, the free molecule regime limit - Equation (2.36) - can be used to calculate the particle diffusion coefficient.

\subsubsection{Nondimensional Formulation}

For generality, Equation (2.33) can be nondimensionalized by choosing a characteristic length (taken here as $S$, the distance between the showerhead and wafer), velocity (taken here as $U_{o}$, the mean inlet velocity of the flow at the showerhead), and concentration $\left(n_{o}=\Lambda_{h} / U_{o}\right.$, the trap source strength divided by inlet velocity, as discussed in Chapter 5). For steady state, and assuming a constant diffusion coefficient, Equation (2.33) can be written (Peters et al., 1989):

$$
\tilde{U} \cdot \nabla \tilde{n}=\frac{1}{\operatorname{Pe}} \nabla^{2} \tilde{n}-\nabla \cdot\left(\tilde{V}_{p}^{t} \tilde{n}\right)+1
$$


where the Peclet number is a dimensionless number defined as:

$$
\operatorname{Pe}=\frac{S U_{o}}{\mathcal{D}}
$$

The Peclet number is a measure of the relative strengths of convective and diffusive particle transport; large Pe values indicate that transport is controlled by convection, while small Pe values indicate that transport is controlled by particle diffusion. The continuum regime limit for the Peclet number is:

$$
\mathrm{Pe}_{\text {continuum }}=\frac{3 \pi \mu d_{p} S U_{o}}{k T}
$$

which is proportional to particle diameter. Thus, in the continuum limit, the Peclet number can be used a dimensionless particle diameter.

In the free molecular limit:

$$
\mathrm{Pe}_{\text {molecular }}=\frac{3 \phi}{(\alpha+\beta)}\left(\frac{2 \pi M}{R T}\right)^{\frac{1}{2}} \frac{P d_{p}^{2} S U_{o}}{k T}
$$

which is proportional to particle diameter squared and to pressure. In the free molecular limit, the square root of the Peclet number can be used as a dimensionless particle diameter.

In standard problems of species mass transfer, the Peclet number would be sufficient to completely characterize the problem for a given geometry and flow field. For particles, however, the presence of a drift velocity term (the second term on the right hand side) means that the Peclet number no longer uniquely specifies the solution and a dimensionless drift velocity ratio must also be considered:

$$
\tilde{V}_{p}^{t}=\frac{V_{p}^{t}}{U_{o}}
$$


Blank Page 


\section{Analysis of Fluid Flow in a Parallel Plate Reactor}

In this chapter, we first present the assumptions used to describe the fluid transport in a showerhead/ wafer parallel plate process tool. Next, the equations governing fluid transport are presented; nondimensionalization of the governing equations is also discussed. The problem is separated into two domains: flow through the showerhead and flow between two parallel plates. The flow field in each domain is solved based on the simplifying assumptions presented below.

\subsection{Problem Description}

The axisymmetric geometry we consider consists of a showerhead separated by a finite gap, $S$, from a parallel wafer, as shown in Figure 1. The radius and diameter of the wafer (and showerhead) are $R_{W}$ and $D_{W}$. The showerhead consists of a thickness, $L$, of a material through which $N_{j e t}$ straight-sided holes of diameter $d_{j e t}$ are drilled (see Figure 1). The holes are assumed to be small, numerous, and uniformly distributed over the showerhead. A volumetric gas flow $Q$ is forced to pass through the showerhead and into the region between the showerhead and wafer, where the flow is turned and allowed to exit the domain radially.

Several general assumptions used throughout the analysis are listed and described below. Additional assumptions are discussed as needed in the fluid and particle analysis sections.

1. Gas flow is incompressible, laminar, and steady. Incompressibility is satisfied because typical gas velocities in process tools are much less than the speed of sound. At the low operating pressures typical of commercial process tools, low gas densities result in low Reynolds numbers that are well within the laminar regime. The steady flow assumption prevents extending the current analysis to process transients which affect the gas flow, such as pressure and/or flow changes, although the particle source is not required to be steady.

2. Constant gas properties. Temperature-dependent variations in gas properties are neglected, limiting applicability of the present results to the case where variations in gas properties are small relative to their mean values. Modest temperature gradients are allowed as a driving force for particle thermophoresis.

3. Inlet flow is uniform injection through a porous plate. The showerhead is assumed to act as a porous plate by evenly distributing flow across its face, thereby producing a uniform flow characterized by a mean axial (or face) velocity of magnitude $U_{o}=Q / \pi R_{W}{ }^{2}$ that does not vary in the radial direction. Implicit in this assumption is that the total flow is evenly divided among the showerhead holes, and that the individual jets effusing from the showerhead merge immediately. Any persistence of the jets into the gap would give particles a longer time to accelerate (equivalent to increasing the showerhead thickness) and less time to de-accelerate (equivalent to decreasing the separation between the showerhead and wafer). Thus, jetting effects would act to increase inertial deposition relative to what is predicted below.

4. Gas velocity and particle flux are independent of axial position in each showerhead hole. This assumption neglects the details of the flow upstream of the showerhead and in the entrance region of the hole where both the fluid and suspended particles are accelerated as the flow field develops. Entrance effects would give particles a longer time to accelerate (equivalent to increasing the showerhead thickness) and would focus the particles toward the centerline; both effects would act to increase inertial deposition relative to what is predicted below.

5. Flow in the showerhead holes is laminar and streamlines lie parallel to the walls. Typically, commercial tools operate with showerhead-hole Reynolds numbers (based on hole diameter) that are less than several hundred; laminar flow is expected for Reynolds numbers as high as 2100. Further discussion of the second assumption is contained in the showerhead flow analysis section. 
The previous assumptions may prove to be too restrictive in the analysis of real process tools; e.g., when strong temperature gradients are present, when jetting phenomena become prominent, or when the tool geometry becomes complex. In these cases numerical solutions of coupled thermal and fluid flow must be sought. For the infinite parallel plate geometry, Coltrin et al. (1991) have developed the Fortran program SPIN for modeling one-dimensional coupled fluid and thermal transport. In addition to the velocity field, the code calculates the axial variation of temperature ${ }^{1}$ and temperature-dependent gas properties (such as density and viscosity). For coupled fluid/thermal transport in more complex geometries, several commercial codes are available; the results of one of these codes $\cdot$ is presented below. Particle transport in a variable temperature, complex-geometry system can still be described by the analysis of Chapter 2, although the spatial variation of gas properties (through their temperature dependencies) must now be included in the integrations.

\subsection{Fluid Transport Equations}

In both of the domains considered (flow within the showerhead and between two parallel plates), the geometry will be axisymmetric. Under the above assumptions (constant properties, incompressible; laminar, steady flow) the governing equations for axisymmetric flow are the conservation of mass:

$$
\frac{\partial u}{\partial z}+\frac{1}{r} \frac{\partial}{\partial r}(r v)=0
$$

and conservation of momentum:

$$
\begin{gathered}
\rho\left(\frac{\partial v}{\partial t}+v \frac{\partial v}{\partial r}+u \frac{\partial v}{\partial z}\right)=-\frac{\partial P}{\partial r}+\mu\left(\frac{\partial^{2} v}{\partial r^{2}}+\frac{1}{r} \frac{\partial v}{\partial r}+\frac{\partial^{2} v}{\partial z^{2}}-\frac{v}{r^{2}}\right) \\
\rho\left(\frac{\partial u}{\partial t}+v \frac{\partial u}{\partial r}+u \frac{\partial u}{\partial z}\right)=-\frac{\partial P}{\partial z}+\mu\left(\frac{\partial^{2} u}{\partial r^{2}}+\frac{1}{r} \frac{\partial u}{\partial r}+\frac{\partial^{2} u}{\partial z^{2}}\right)
\end{gathered}
$$

where $u$ and $v$ are the axial and radial components of the fluid velocity, $P$ is the pressure, $\rho$ is the fluid density, and $\mu$ is the fluid viscosity (Bird et al., 1960, p. 85).

Boundary conditions are needed to complete the problem specification: 1) no-slip (zero radial and axial velocities) is assumed at solid walls (i.e., on the wafer, and walls of the showerhead holes), 2) symmetry is assumed along centerlines (zero radial velocity and zero normal derivative of the axial velocity), and 3) zero gradients are assumed at all outflows. For the two flow domains, specific inlet boundary conditions and methods for solving the governing equations are discussed in greater detail below. Note that for convenience, a different coordinate system is used for describing the flow through a showerhead hole than in the region between the parallel plates (see Figure 1b).

The generality of the results are improved if the fluid equations are solved in nondimensional form. Because there are two domains of interest (flow within the showerhead and between the plates), there are two choices for a characteristic length and velocity. For flow in the showerhead holes, the hole diameter $d_{j e t}$ and the magnitude of the mean velocity $\bar{U}_{j e t}$ are used as the characteristic length and velocity, for which the jet Reynolds number is defined as $\operatorname{Re}_{j e t}=\rho \bar{U}_{j e t} d_{j e t} / \mu$. For the flow between two plates, the inter-plate separation $S$ and the magnitude of the mean face velocity $U_{o}$ are the appropriate choices, and the inter-plate flow is then characterized by a separate Reynolds number: $\operatorname{Re}=\rho U_{o} S / \mu$.

\footnotetext{
${ }^{1}$ For the infinite parallel plate geometry, temperature is found to be independent of radial position.
} 


\subsection{Fluid Transport in the Showerhead Holes}

The idealized geometry and the coordinate system for fluid and particle transport in the showerhead holes is shown in the insert in Figure 1b. Unlike within the reactor, $z$ is taken as increasing in the direction of flow (towards the wafer), so that all fluid and particle axial velocities considered in this part of the solution are positive.

The flow in the showerhead holes is assumed laminar with parallel streamlines, thereby neglecting any axial variations in velocity. For laminar flow in a tube with a uniform inlet velocity, however, it is well known that a fully developed parabolic velocity profile develops over an entrance length given approximately by $0.04 d_{j e t} \operatorname{Re}_{j e t}$ (Friedlander, 1977, p. 72). For many showerheads this entrance length is much less than the hole length (showerhead thickness) and so may be safely neglected; for thin showerheads, however, this may not be the case. In the present analysis, we consider two limiting velocity profiles that meet the above assumptions: 1) plug flow (constant velocity profile) and 2) fully-developed laminar flow (parabolic velocity profile). For laminar flow, the velocity profile anywhere along the hole will fall somewhere between these two limiting cases. In case one, the velocity is constant throughout the tube and, for incompressible flow, is equal to the mean velocity in the hole, $\bar{U}_{j e t}=4 Q /\left(N_{j e t} \pi d_{j e t}^{2}\right)$. By mass conservation it can be shown that the ratio of the mean axial velocity in a hole and the face velocity is the ratio of the showerhead area to the total hole area:

$$
\frac{\bar{U}_{j e t}}{U_{o}}=\frac{A_{\text {showerhead }}}{\Sigma A_{j e t}}=\frac{D_{W}^{2}}{N_{j e t} d_{j e t}^{2}} .
$$

For case two, fully-developed laminar flow in a tube is given by:

$$
U_{j e t}(r)=2 \bar{U}_{j e t}\left(1-\left(\frac{r}{a_{j e t}}\right)^{2}\right)
$$

where $r$ is the radial distance from the tube centerline and $a_{j e t}$ is the radius of the hole. The maximum velocity for parabolic flow is twice the mean velocity and occurs on the centerline.

\subsection{Fluid Transport between Parallel Plates}

Both analytic and numerical techniques have been used to calculate the flow between the showerhead and wafer. For both methods we assume axisymmetric, incompressible, constant property, laminar, steady flow between two parallel plates (Figure 1b). Note that here $z$ is zero at the wafer. The flow enters through the showerhead $(z=S)$ and is assumed to spread immediately, so that the inlet boundary condition is assumed to be a uniform axial velocity, $-U_{o}$, with zero radial velocity. Both the radial and axial components of velocity vanish at the lower plate $(z=0)$. The analytic approach assumes that the plates are infinite in the radial direction and that the Reynolds number is small; the numerical technique is used for finite plates and is valid for higher Reynolds numbers. The numerical technique is relied on here to define the conditions over which the simpler analytic solution is valid.

The assumption of constant-property flow requires further comment. Although the numerical methods used here can solve for coupled fluid-thermal transport, we have not used this capability in the present analysis. For cases where temperature differences are large or where more accurate solutions are needed for a specific application, the reader is advised to perform the coupled fluid-temperature problem. As mentioned above, a modest temperature gradient is allowed to drive particle thermophoresis (the magnitude of all particle drift velocities will be calculated using fluid properties evaluated at the wafer temperature).

Under the above assumptions, the flow between the plates is entirely determined by the geometry and the Reynolds number $\operatorname{Re}=\rho U_{o} S / \mu$. For the range of conditions encountered in semiconductor reac- 
tor processes, the associated Reynolds numbers are typically less than one, and seldom greater than ten. For small Reynolds numbers, viscous effects dominate fluid inertial effects and the "creeping flow" or Stokes flow regime is encountered; it is this regime that allows an analytic solution. The numerical technique was used to calculate flow fields for Reynolds numbers up to eight.

\subsubsection{Finite Element Code FIDAP.}

The numerical method used for calculating the fluid velocity field was the commercial fluid dynamics analysis code FIDAP (Version 7, Fluid Dynamics International, Evanston, Illinois, USA). This general purpose finite-element code allows steady-state or transient simulations of fluid and thermal transport in 2-D, 3-D, and axisymmetric geometries. A variety of options for mesh generation are available that allow computations in complex geometries. Here, however, in keeping with our previous assumptions, FIDAP is used to model axisymmetric, laminar, incompressible, constant property, steady flow between two plates. For these simulations, a finite radial domain of radius $R_{W}=12$ was used. ${ }^{2}$ The use of a finite domain requires that an outlet boundary condition be imposed: here a zero traction assumption was made for the flow exiting the domain at $R_{\mathrm{W}}$. All calculations used 9-node, biquadratic quadrilateral elements with a mixed, discontinuous pressure scheme. A quasi-Newton solution algorithm was selected with error tolerances of $1 \times 10^{-5}$. Grid refinement studies were used to verify that converged solutions were being obtained. Typically, calculations were first performed with a coarse, graded mesh of 15 elements ( 31 node points) in the axial direction; next, the number of elements was doubled to 30 (61 node points) and the calculation repeated. The solution was not very sensitive to the number of elements in the radial direction; typically 60 elements were used. The results from a few grid-refinement studies are shown in Figure 6; as can be seen, the coarse and refined mesh results are indistinguishable. For all the cases reported, calculations with the refined mesh agreed with coarse mesh results to four significant figures. The ease with which accurate solutions could be obtained with a fairly sparse mesh results from a combination of the simple geometry, low Reynolds numbers, and use of quadratic elements.

With fixed values for plate separation $(S=1)$, mean inlet velocity $\left(U_{o}=1\right)$, and fluid viscosity $(\mu=1)$, the fluid density $\rho$ was varied to obtain flow field solutions for Reynolds numbers between one and eight. The FIDAP option of solving the Stokes flow equations $(\operatorname{Re}=0)$ was also used. The results of these calculations are given in Figure 7, which shows axial and radial velocity profiles at $r=1$ for $\operatorname{Re}=0,1,2,4$, and 8 as a function of the dimensionless axial coordinate $z / S$. Note that all velocities have been normalized by the magnitude of the inlet velocity $U_{o}$, and that the radial velocity is also normalized by radius. For $\operatorname{Re}=0$, the radial velocity profile is found to be parabolic and symmetric around $z / S=0.5$. As Reynolds number increases, the symmetry vanishes and the maximum in the radial velocity moves closer to the plate $(z / S=0)$. Variations in the axial and radial velocity profiles are seen to be quite small for Reynolds numbers less than two.

As in previous work in a similar geometry (Rader and Geller, 1994), it was found that the flow was quasi-1-D except near the radial edge of the domain $r=R_{W}$. By quasi-1-D, we mean that the axial velocity is independent of radius, while the radial velocity is found to scale with radius such that $v / r$ is independent of radius. Calculations of the radial variation of axial velocity (midway between the plates) are shown in Figure 8 for several Reynolds numbers and plate separations. Inspection of Figure 8 reveals three interesting trends: 1) for small plate separations, the extent of the uniform interior region is only weakly dependent on Reynolds number, 2) for small Reynolds numbers, the extent of the uniform region varies with plate separation but the magnitude of the error does not, and 3) for higher Reynolds numbers, both the extent of the uniform region and the magnitude of the error depend strongly on plate separation. The first observation is supported by comparing the velocity profiles for $R e=1,4$, and 8 all with a plate separation of one (corresponding to an aspect ratio $R_{W} / S=12$ ). In all three cases, the axial velocity is seen to be constant over the majority of the interior out to a ra-

\footnotetext{
2. Arbitrary units are used for all lengths and gas properties; the values are selected to provide the desired Reynolds number.
} 

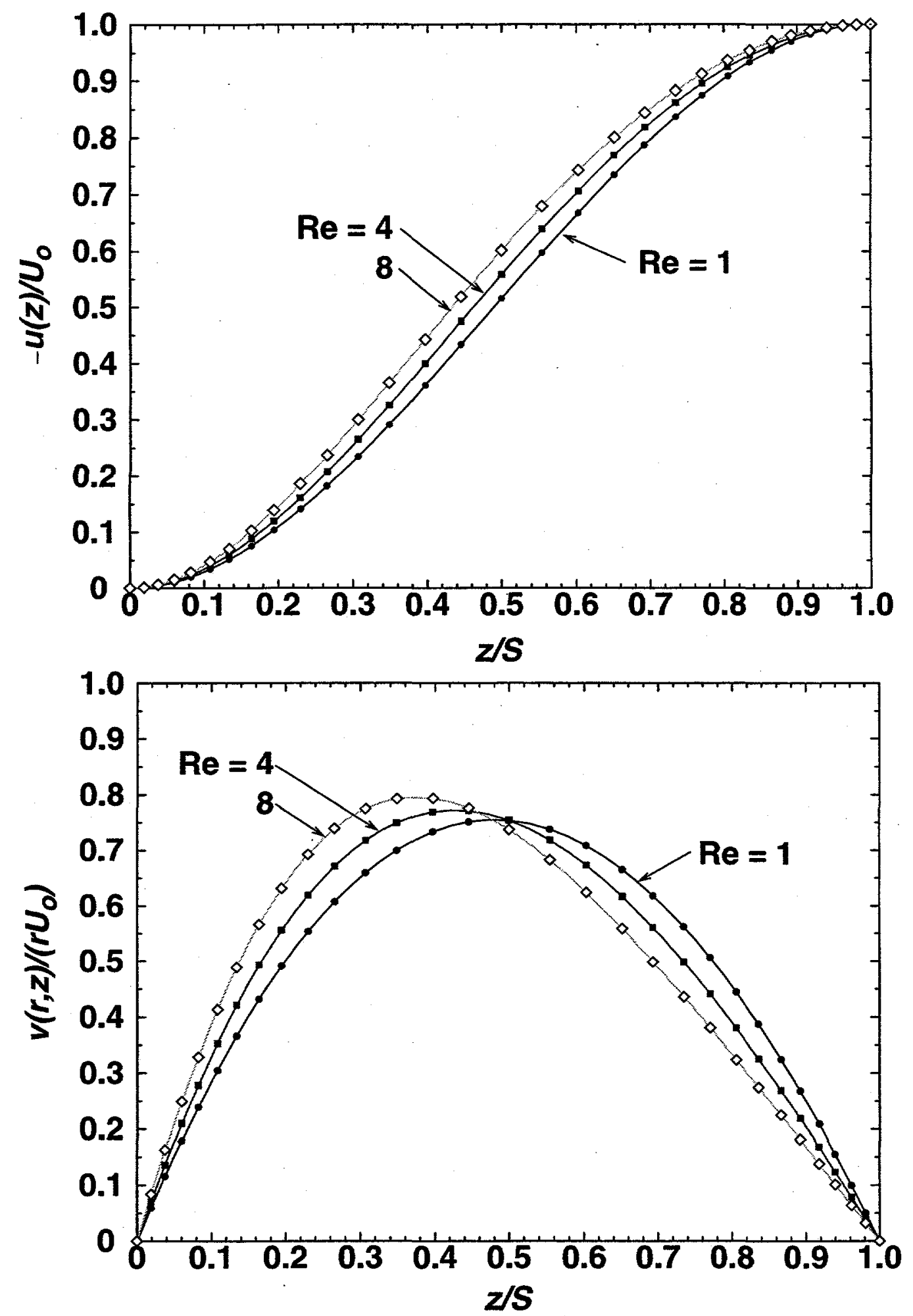

Figure 6. Grid Refinement Studies. Axial and radial velocity profiles for $R e=1,4$, and 8 calculated on a coarse (15 elements) and refined ( 30 elements) mesh. Lines represent the results of the refined mesh, while the symbols give the results of the coarse mesh. ( $r=S=1$ for all curves) 

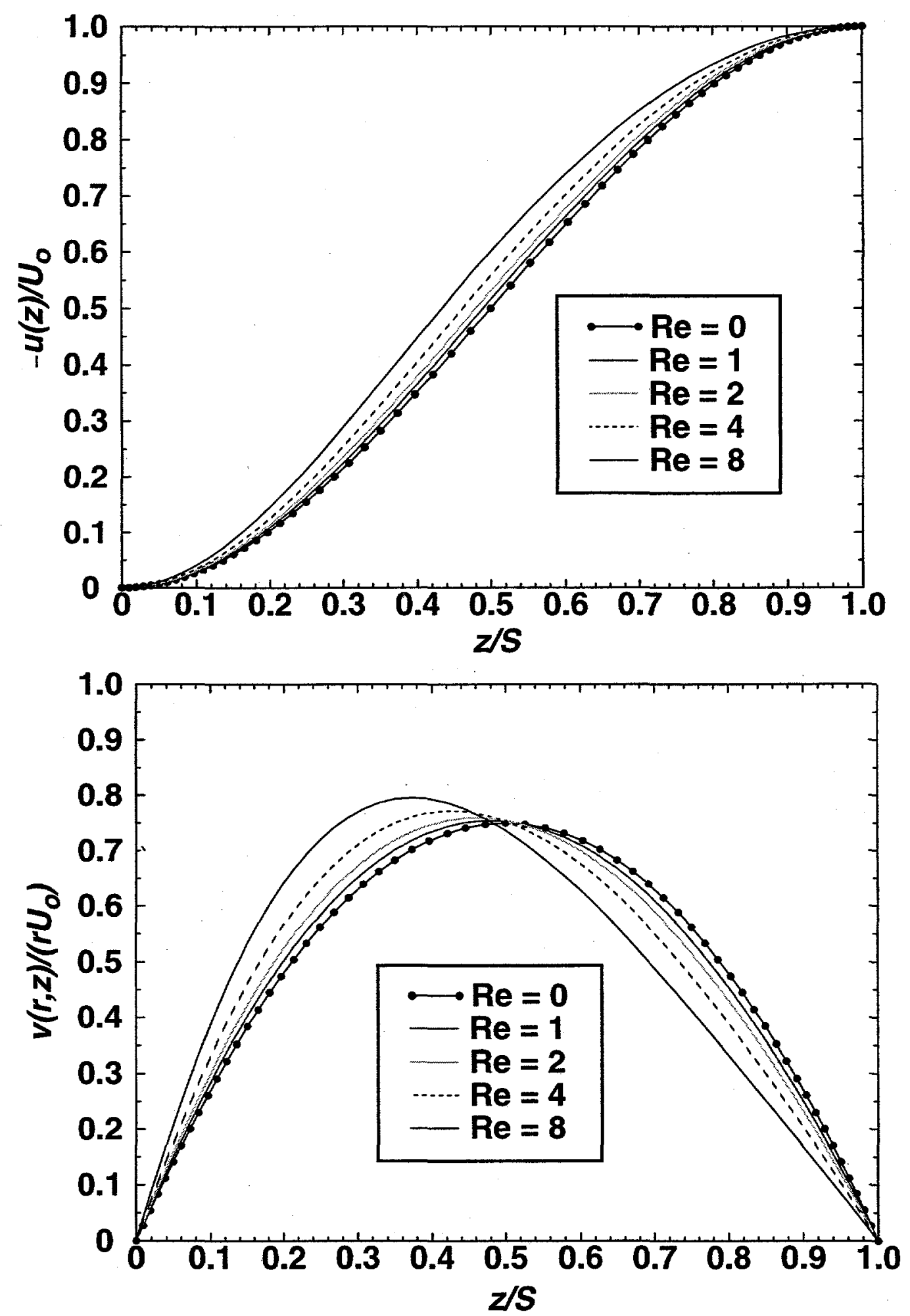

Figure 7. Flow Field Results for Various Re. Axial and radial velocity profiles for Re $=0,1,2,4$, and 8 calculated on a refined ( 30 elements) mesh. ( $r / S=1$ for all curves) 


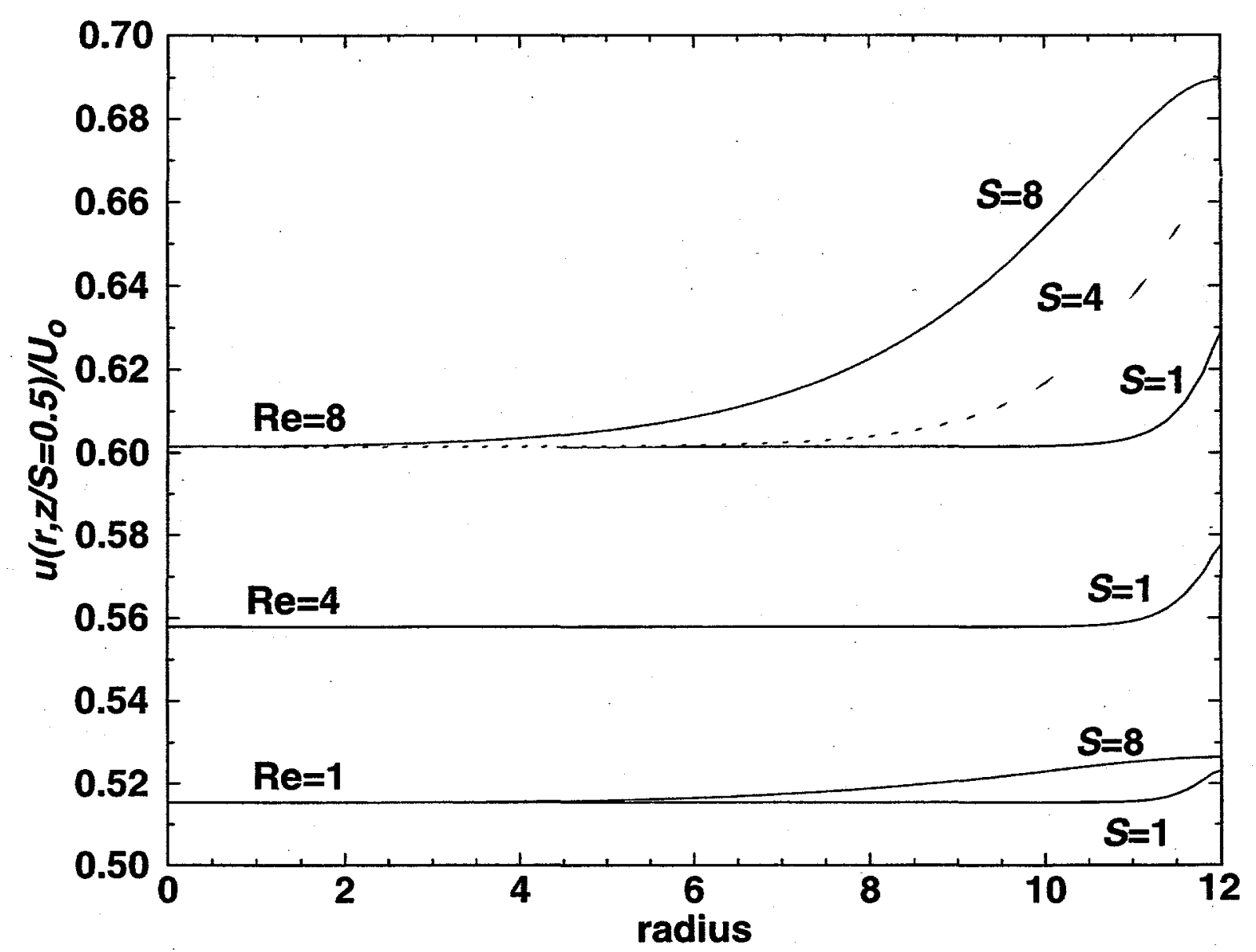

Figure 8. - Edge Effects. Axial velocity at the mid-plane $(z / S=0.5)$ is plotted versus radius for gap spacings $S=1,4$, and 8 at $\operatorname{Re}=8$, for $S=1$ at $\operatorname{Re}=4$, and for $S=1$ and 8 at $\operatorname{Re}=1$ (30x60 element mesh).

dius of about 11; the radial location of the onset of nonuniformity is not noticeably affected by the Reynolds number. The second observation is supported by comparing the velocity profiles for $\operatorname{Re}=1$ with plate separations of 1 and 8. Although the magnitude of the edge effects are roughly the same at the edge, the onset of the nonuniformity begins nearer the centerline for the larger plate separation. The third observation is supported by comparing the velocity profiles for $R e=8$ with plate separations of 1,4 , and 8 (aspect ratios of 12,3, and 1.5). Here, the region of uniform axial velocity retreats towards the centerline while the magnitude of the deviation increases as the plate separation widens.

An interesting result of these edge-effect studies is that, for Reynolds numbers below about eight, the nonuniform region is restricted to an annular region extending about one plate-separation distance radially inward from the wafer's outer edge. Thus, as a gross rule-of-thumb, the flow can be approximated as quasi-1-D in the core region between the centerline and the radial position $\left(R_{W}-S\right)$. This is not generally true, as the geometric and process details of the reactor will determine the precise region over which the 1-D solution is adequate. Further discussion is available in Houtman et al. (1986), who used full thermal/fluid models of a simple stagnation point flow CVD reactor geometry (very similar to the present geometry) to determine the range of operating conditions over which the 1-D treatment accurately predicts the deposition process. Although they were looking specifically at the uniformity of heat transfer, their detailed results qualitatively support the above rule-of-thumb. Also, in unpublished work, the present authors have used finite element simulations of fluid, thermal, and 
particle transport in parallel-plate, single-wafer, commercial CVD and etch process chambers and have found the 1-D approximation to be reasonably accurate over most of the wafer's surface. Particularly for low Reynolds numbers and small aspect ratios, the assumption of 1-D flow over the majority of the inter-plate region is reasonable.

\subsubsection{Analytic.}

A great simplification is gained if the flow between the plates can be approximated as a quasi-1-D stagnation point flow. Terrill and Cornish (1973) give an asymptotic solution to the problem of axisymmetric, laminar, incompressible, constant property and steady flow between two co-axial infinite parallel disks with constant injection across the disks (a uniform gas inlet velocity across the showerhead). Under these assumptions, a similarity solution reduces the 3-D Navier-Stokes equations to a system of ordinary differential equations; for low Reynolds numbers (creeping flow), these equations can be solved with a power series in Reynolds number (Terrill and Cornish, 1973). The first two terms of their asymptotic expansion (translated into the present problem definition) are:

$$
\begin{gathered}
\tilde{u}(\tilde{z})=\frac{u(\tilde{z})}{U_{o}}=2 \tilde{z}^{3}-3 \tilde{z}^{2}+\frac{\operatorname{Re}}{70}\left(2 \tilde{z}^{7}-7 \tilde{z}^{6}+18 \tilde{z}^{3}-13 \tilde{z}^{2}\right) \\
\tilde{v}(\tilde{r}, \tilde{z})=\frac{v(\tilde{r}, \tilde{z})}{U_{o}}=\tilde{r}\left[3 \tilde{z}-3 \tilde{z}^{2}-\frac{\operatorname{Re}}{70}\left(7 \tilde{z}^{6}-21 \tilde{z}^{5}+27 \tilde{z}^{2}-13 \tilde{z}\right)\right]
\end{gathered}
$$

where $\tilde{z}=z / S$ and $\tilde{r}=r / S$. These two equations exactly satisfy all boundary conditions. The quasi-1-D nature of the result is clearly seen as the axial velocity is independent of radius, while the radial velocity scales linearly with radius.

In the limit of vanishingly small Reynolds number, Equation (3.5) reduces to a symmetric, parabolic profile for radial velocity, in excellent agreement with the Stokes flow solution $(\operatorname{Re}=0)$ obtained by FIDAP (this limit has also been previously reported by Houtman et al., 1986). To determine the range of Reynolds numbers over which the asymptotic solution is accurate, velocity profile calculations using Equation (3.5) have been compared to the FIDAP simulations reported in the previous section. ${ }^{3}$ The error in the asymptotic solution is defined as the ratio of the velocity calculated by Equation (3.5) to the numerical result calculated by FIDAP (with the refined 30x60 mesh, with $S=1$ and $U_{o}=1$ ); the error for both axial and radial velocity are shown in Figure 9. Equation (3.5) does a very good job of approximating the axial velocity profile - agreeing with FIDAP solutions to better than $1 \%$ for Reynolds numbers less than 4 , and to better than about $4 \%$ for Reynolds numbers up to 8 . The success of Equation (3.5) in predicting radial velocity is not nearly so good. Although the error is better than $1 \%$ for $\operatorname{Re}<1$, the maximum observed error quickly grows, reaching $15 \%$ for $\operatorname{Re}=4$ and $70 \%$ at $\operatorname{Re}=8$. As can be seen, the largest errors are found near the showerhead $(z / S=1)$, where the magnitude of the radial velocity is quite small. Thus, although the relative error is quite large, the absolute error is small. In any case, our treatment of the region near the showerhead is only approximate because we have neglected the effect of the discrete jets issuing from the showerhead. Strictly from a fluid velocity point of view, Equation (3.5) provides a very good approximation of the flow for Reynolds numbers less than two, and a reasonable approximation up to a Reynolds number of four. The utility of the analytic solution in describing particle transport is discussed in later chapters.

\footnotetext{
3. Since Equation (3.5) is 1-D, its results must be compared with FIDAP calculations in the central region of the domain (from Figure 8, all FIDAP solutions are essentially 1-D for $r<1$ ).
} 

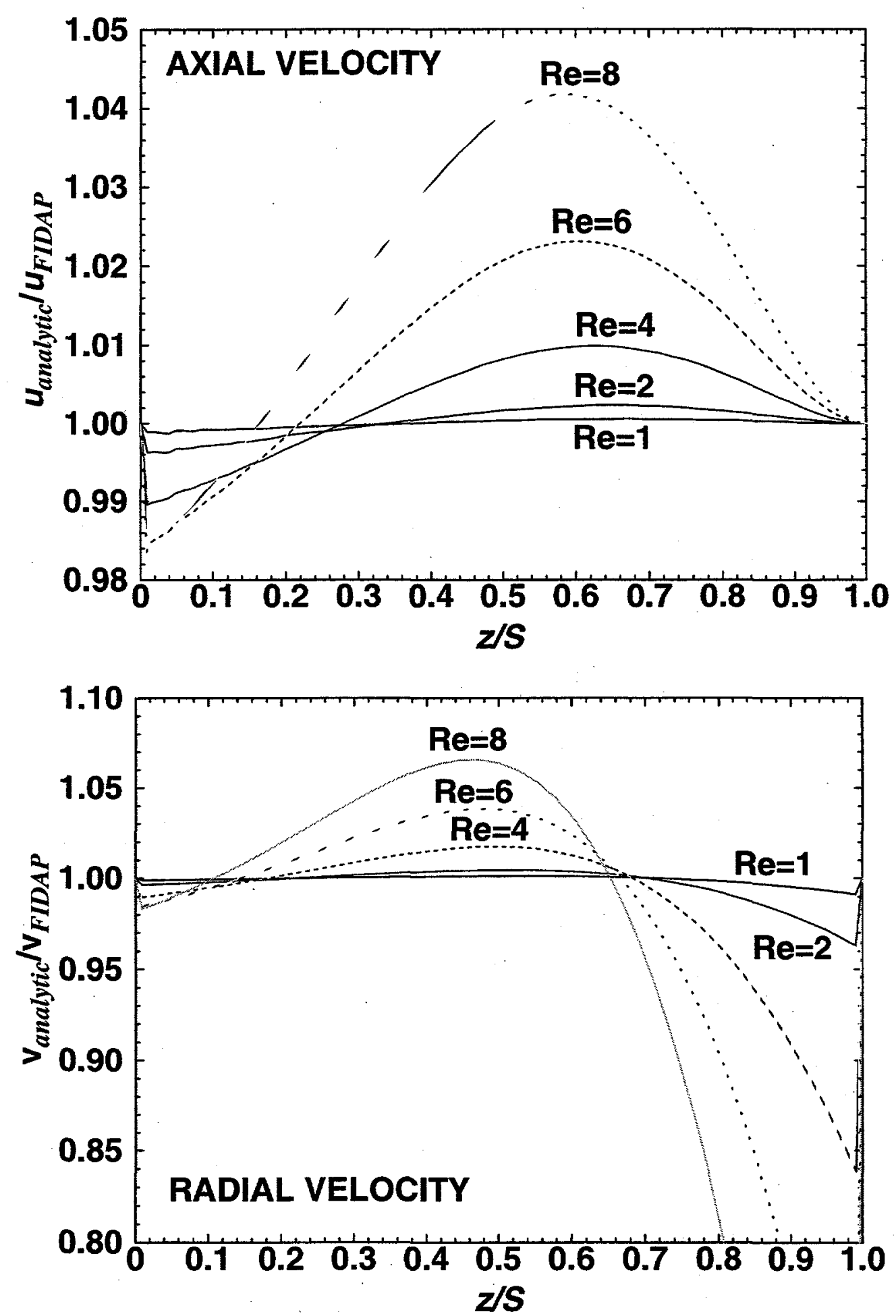

Figure 9. Comparison of 1- and 2-D Solutions. The ratio of the 1-D analytic to the 2-D FIDAP numerical solution are shown for both the axial and radial velocity. Results for $\operatorname{Re}=1,2,4,6$, and 8 are shown. The FIDAP solution used a refined mesh (30x60 element) with a gap spacing $S=1$; the profile was taken at $r=1$, where edge effects are small. 


\subsection{Summary}

This chapter has defined the parallel-plate geometry which will be used to approximate the flow inside a showerhead-type etch or CVD reactor. The acceleration of the gas flow as it passes through the showerhead will later be found to play a key role in enhancing particle deposition by particle inertia; for this reason, solutions for flow within the showerhead holes have been presented. The two limiting cases which were considered, plug and fully-developed parabolic flow, should bracket the range of flows likely to be encountered in semiconductor applications. Laminar, incompressible, constant-property flow between two infinite, parallel plates is used to approximate the inter-plate flow in real reactors which are certainly more complicated. Reynolds number and edge effects were discussed in the chapter, and an analytic solution was found that should provide a fairly accurate description of the flow for Reynolds numbers less than about four when the plate separation is much smaller than the overall system radial dimension. Variable temperature effects have not been considered in this work. 


\section{Particle Collection Efficiency}

The collection efficiency is defined as the fraction of particles present in the inter-plate region that deposit on the wafer. The collection efficiency is introduced to provide a single parameter that can be used to compare particle transport and deposition results among many cases. Note that the use of collection efficiency side-steps the important issue of the particle source term. Thus, while the present transport analysis addresses the question of the fraction of gas-borne particles that deposit on the wafer, a prediction of the number of particles that deposit on the wafer additionally requires a clear understanding of the controlling particle generation mechanisms. In practical terms, the present analysis helps identify strategies for reducing the probability that particles are transported to and deposit on a wafer; a complete strategy for reduction of total particle-on-wafer counts also requires that particle source terms be understood and controlled.

In this work, three particle-source scenarios are considered: 1) a continuous source of particles entering the inter-plate region through the showerhead with known concentration (such as for contaminated process gases), 2) a discrete number of particles that are originally trapped between the plates (such as by a plasma) but are subsequently released (such as at plasma extinction), and 3) a continuous source of particles which are created between the plates at a known generation rate (such as by particle nucleation). General collection efficiency expressions for these cases are defined below. In addition, analytic expressions are provided for the limiting case where external forces control particle deposition - i.e., both particle inertia and Brownian motion are neglected.

\subsection{External Force Limit}

In the absence of particle inertia and Brownian motion, Robinson (1956) has shown that particle concentration is constant along particle trajectories if: 1) the flow is incompressible, and 2) the external forces acting on the particle are all divergence free. For the infinite parallel-plate geometry with constant-property flow, the flow is clearly incompressible and the second condition is met for the gravitational and Coulombic electric particle forces (which are each constant between the plates). When the flow is not isothermal, however, temperature dependencies in local gas properties result in all particle forces having a nonvanishing divergence. ${ }^{1}$ Moreover, the divergence of the thermophoretic force, which exists only in the presence of a temperature gradient, is always nonzero. Thus, strictly speaking, the efficiency expressions presented in this section for the external-force limit only apply for isothermal flow and do not rigorously apply for thermophoresis. However, in this work, we will often apply the isothermal approximation even in the case of modest temperature differences between the plates as long as the resulting particle external forces (including thermophoresis) are nearly constant, and the flow field is satisfactorily incompressible.

For the present 1-D geometry, where the particle concentration boundary condition is assumed radially uniform, the Robinson result implies (assuming negligible inertia and Brownian motion) that the particle concentration will be uniform everywhere above the lower plate. ${ }^{2}$ In this limit, the calculation of particle deposition onto the lower plate is quite simple - being the product of the net particle drift velocity (drift velocity normal to the lower plate induced by summing over all external forces) times the concentration at the showerhead. Interestingly, in the regime where external forces dominate, particle defect density should be uniform over the wafer surface (i.e., no radial dependence) assuming a radially uniform particle source.

\footnotetext{
1. Temperature variability also implies that the flow becomes compressible.

2. Robinson's result that particle concentration is constant along particle trajectories does allow for concentration discontinuities. For example, consider a uniform concentration of particles starting from a finite region about the showerhead centerline, while the remainder of the showerhead flow was clear of particles. In this 2-D case, regions that held particles would be characterized by a uniform concentration, while adjacent particle-free regions would be characterized by zero concentration. For our 1-D geometry, however, concentration discontinuities can only exist in the axial direction, i.e. particle traps or clear zones.
} 


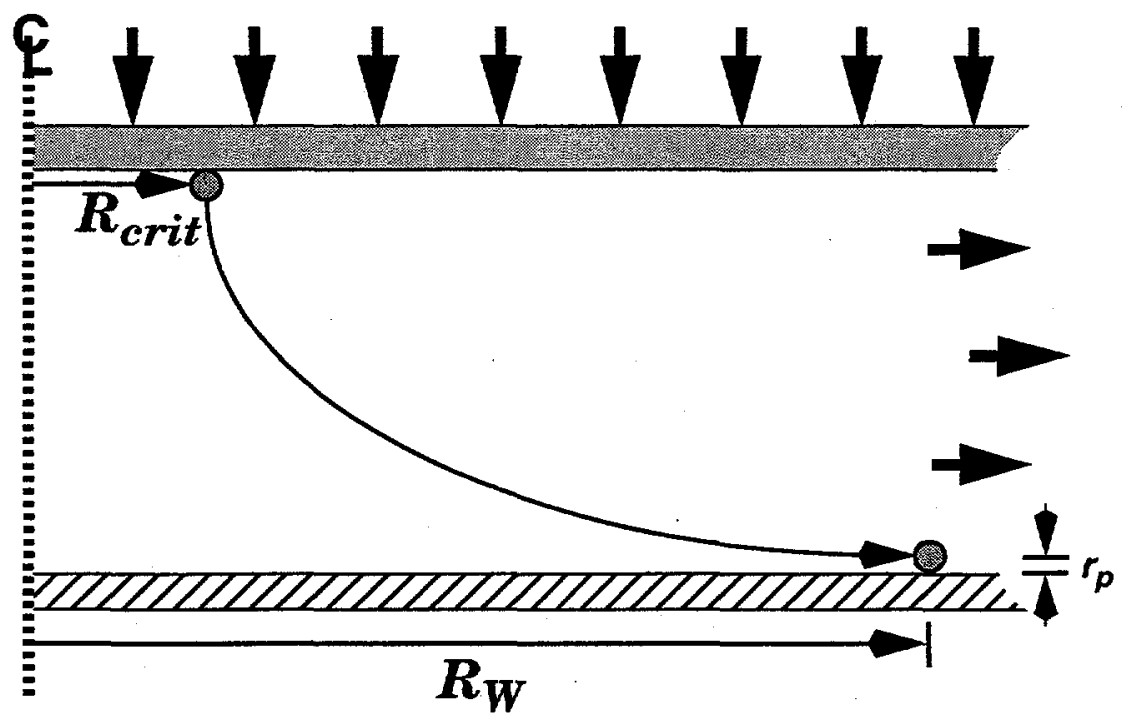

Figure 10. Critical Trajectory. Diagram of a critical trajectory for a particle which starts at the showerhead at radial position $R_{c r i t}$ and deposits at the wafer edge $R_{W}$.

\subsection{Particles Entering Through the Showerhead}

For this case, collection efficiency is defined as the fraction of particles entering the inter-plate region through the showerhead (between $r=0$ and $R_{W}$ ) that deposit on the wafer. These particles are presumed to originate upstream of the showerhead and are assumed to be evenly distributed across the showerhead. Particle acceleration through the showerhead needs to be considered in this case, which is treated by a Lagrangian formulation in Chapter 6 . Note that the present Lagrangian formulation accounts for the coupling between particle inertia and external forces in determining particle transport in the inter-plate region. The calculation of a collection efficiency with a Lagrangian technique requires the determination of the critical radius, $R_{\text {crit }}$, which is the starting radial position (at the showerhead) of a particle that follows a trajectory that leads it to deposit at the edge of the wafer, $R_{W}$ (see Figure 10). All particles starting closer to the centerline will deposit on the wafer, while those starting farther out will exit the reactor. For a uniform concentration across the showerhead, the collection efficiency, $\eta$, can be written as:

$$
\eta=\left(\frac{R_{c r i t}}{R_{W}}\right)^{2}=\left(\frac{R_{i}}{R_{f}}\right)^{2} .
$$

In general, the critical trajectory must be found by a trial-and-error method. The second equality of Equation (4.1) is a simplification that only applies under our quasi-1-D approximation. In this case, all the factors that influence particle deposition (e.g., axial fluid velocity profile, the particle initial velocity, and particle axial drift velocity) are independent of radial position; thus, the question of whether a particle will hit the wafer must not depend on its initial radial position, $R_{i}$, (although the radial position at which the particle hits the wafer, $R_{f}$, will depend on $R_{i}$ ). It can be shown for our quasi-1-D case that the ratio $R_{i} / R_{f}$ is independent of initial radial position. Thus, efficiency in the Lagrangian framework is calculated by starting the particle at a particular radial position $\left(R_{i}=1\right)$ and calculating its trajectory to determine the radial position of contact with the wafer; ${ }^{3}$ the efficiency is then $\left(R_{i} / R_{f}\right)^{2}=\left(1 / R_{f}\right)^{2}$ as given in Equation (4.1). The total number of particles depositing on the wafer is the product of efficiency times the total flux of particles entering through the showerhead.

\footnotetext{
3. In the event that the particle axial velocity becomes zero, or begins to move away from the wafer, then the trajectory calculation is terminated and the efficiency is set to zero.
} 


\subsubsection{External Force Limit}

For the case where external forces control particle deposition (neglecting inertia, interception, and diffusion), Rader et al. (1994a,b) used a Lagrangian analysis to obtain the following expression for deposition efficiency in isothermal, quasi-1-D parallel-plate flows:

$$
\eta=\left(\frac{R_{c r i t}}{R_{W}}\right)^{2}=\left(\frac{R_{i}}{R_{f}}\right)^{2}=\frac{\left|V_{p}^{t}\right|}{\left|V_{p}^{t}\right|+U_{o}} \quad V_{p}^{t} \leq 0
$$

where $V_{p}{ }^{t}$ is the $z$-component of the net particle drift velocity (the resultant of all external forces in the axial direction). ${ }^{4}$ For net drift velocities greater than zero (net external force pushing particles away from the wafer), no particle deposition on the wafer is predicted (although it will be shown later that particle inertia or diffusion can cause deposition even in this case). Equation (4.2) provides a lower bound for particle deposition, as inertial and diffusional effects can only increase deposition from what is predicted. Interestingly, as the particle net drift velocity is typically much smaller than the fluid entrance velocity, Equation (4.2) predicts that (in the absence of inertia and diffusion) the particles which land on the wafer originate from near the reactor centerline.

That Equation (4.2) is independent of the flowfield (consider that the flow Reynolds number does not appear) can be more easily understood by applying Robinson's (1956) result (as discussed above). Neglecting diffusion and inertia, the concentration over the lower plate must equal the inlet concentration, $n_{o}$. The rate at which particles deposit on the lower plate becomes $\left|V_{p}^{t}\right| n_{o} \pi R_{W}^{2}$, while the rate at which particles enter through the showerhead is $\left(\left|V_{p}\right|+U_{o}\right) n_{o} \pi R_{W}^{2}$. Taking the ratio of these expressions gives the same efficiency as Equation (4.2) (see also Cooper et al., 1990). Similar results, including Equation (4.2), were found by Ramarao and Tien (1989) for plane-stagnation flow.

Interception effects were neglected in Equation (4.2), which implies that a particle is collected only when its center of mass reaches the wafer surface. A better assumption is that particle collection occurs when the particle comes within one particle radius $\left(z=r_{p}=d_{p} / 2\right)$ of the wafer surface. ${ }^{5}$ The derivation of Equation (4.2) can be easily modified to include interception, with the following result:

$$
\eta=\left(\frac{R_{c r i t}}{R_{W}}\right)^{2}=\left(\frac{R_{i}}{R_{f}}\right)^{2}=\frac{\left|V_{p}^{t}\right|+\left|u\left(r_{p}\right)\right|}{\left|V_{p}^{t}\right|+U_{o}} \quad V_{p}^{t} \leq\left|u\left(r_{p}\right)\right|
$$

Note that particle collection is now expected in the absence of external forces (or even for weak repulsive forces); the physical interpretation is that collection occurs when the flow brings the particle within one particle radius of the wall. The inclusion of interception also has the effect that Equation (4.3) (unlike the previous equation) depends on the flow field through the term $u\left(z=r_{p}\right)$. Because the gas velocity one particle radius away from the wafer is typically vanishingly small, interception effects are generally neglected in the following discussion, and Equation (4.2) is used. The numerical Lagrangian trajectory algorithms described in Chapter 6, however, do account for particle interception; thus, for nondimensional studies the influence of interception is numerically eliminated by setting the dimensionless particle diameter to a small value. Dimensional plots presented in Chapter 6 , however, do include interception.

\footnotetext{
4. When inertia is neglected, the particles enter the reactor with the mean gas velocity so that $V_{p o}=-U_{o}$. Also, note that $U_{o}$ is the magnitude of the face velocity, and so is positive.

5. The inclusion of particle interception effects is somewhat overkill, as we have neglected wafer surface roughness/structure which is likely characterized by dimensions similar to particle sizes.
} 


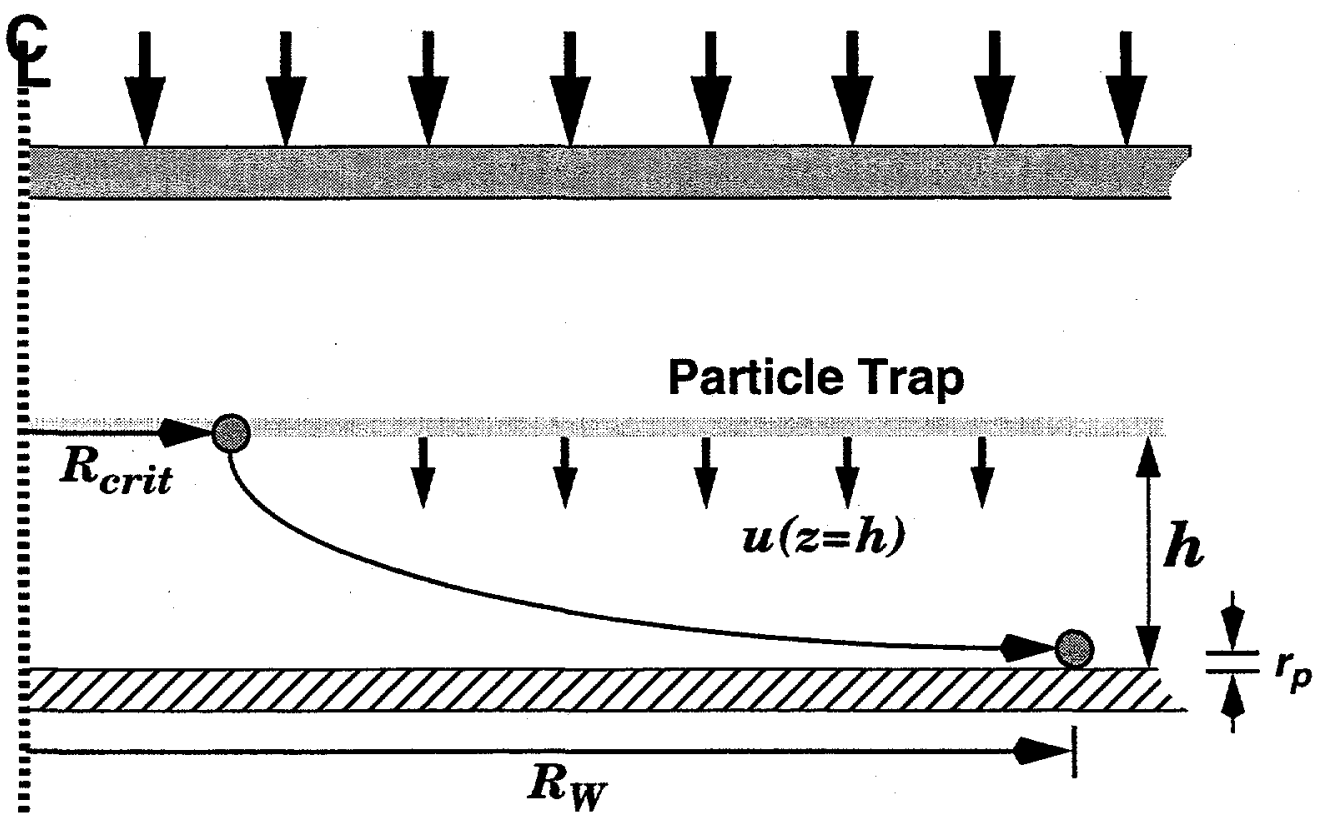

Figure 11. Trap Schematic. Diagram of particles in a planar trap located a distance $h$ from the lower plate; a critical trajectory is also shown for a particle which starts at radial position $R_{c r i t}$ and deposits at the wafer edge $R_{W}$.

\subsection{Particle Traps}

Another source of wafer contamination is from particles that start somewhere between the plates, and are subsequently transported to the wafer. One example is particles generated in situ by nucleation. A second example is particles that are originally trapped between the plates during a plasma process, which are subsequently released at plasma extinction. While the plasma is on, contaminant particles generally accumulate in specific regions of the radio frequency (RF) discharge. Roth et al. (1985) first used laser light scattering to observe that particles accumulate near the bulk plasma-sheath boundary in these discharges. Sommerer et al. (1991) and Barnes et al. (1992) first proposed that particle transport in the discharge is dominated by two forces: electrostatic and viscous ion drag. The electrostatic force accelerates negatively charged particles towards the center of electropositive plasmas, while viscous ion drag accelerates particles in the direction of net ion flux (generally towards plasma boundaries). Particle "traps" occur in regions where the sum of forces acting on the particle vanishes. In many cases these traps are approximately planar and parallel to the plates (Choi et al., 1994); a schematic of a planar trap is shown in Figure 11 where the particles are uniformly distributed at a distance $h$ above the lower plate. Only planar traps are considered in this work, although a variety of other trap structures (rings, domes, etc.) are well known in the literature. For any trap structure more complicated than an infinite plane the problem becomes inherently $2-\mathrm{D}$, which is beyond the scope of the present analysis.

At the end of the process step, the discharge is extinguished and the plasma-induced forces responsible for particle trapping are assumed to dissipate rapidly (compared to particle transport times) in the afterglow. In this work, we assume that the charged particles are rapidly neutralized after the plasma extinction and can therefore be treated as neutral particles; this was experimentally observed by Jellum et al. (1991), Shiratani et al. (1991) and Yeon et al. (1995). ${ }^{6}$ Under the assumption of rapid neutralization, the particles are released from the traps and can deposit on the wafer as a result of

\footnotetext{
6. However, a recent study by Collins et al. (1996) suggests that some particles might retain a few residual charges (positive or negative) in the afterglow.
} 
external forces, inertia, or Brownian motion (diffusion). To analyze the extent of deposition both the Lagrangian and Eulerian formulations have been used. The Lagrangian formulation allows simultaneous consideration of external forces and inertia, although particle diffusion is neglected; the Eulerian approach allows simultaneous consideration of external forces and diffusion, although inertial effects are neglected. Although the physical interpretation of efficiency (fraction of particles starting in the trap that end up on the wafer) is the same for both approaches, the methods of calculating the efficiency are quite different.

\subsubsection{Efficiency for the Lagrangian Formulation}

In the Lagrangian formulation, Brownian motion is neglected and calculation of particle trajectories is determined from the coupling between particle inertia and external forces. Consequently, the determination of a collection efficiency reduces to the determination of a critical trajectory just as defined in Equation (4.1) of the previous section, except that the particle starting position is now at axial position $h$ and the particle initial velocity is assumed to be zero. As before, it can be shown for our quasi-1$\mathrm{D}$ case that the ratio $R_{i} / R_{f}$ is independent of initial radial position within the trap. Thus, efficiency in the Lagrangian framework is calculated by starting the particle at a particular radial position $\left(R_{i}=1\right)$ in the trap $(z=h)$ and calculating its trajectory to determine the radial position of contact with the wafer; the efficiency is then calculated by Equation (4.1).

\subsubsection{Efficiency for the Eulerian Formulation}

For small particles and/or at low pressure, the effects of Brownian motion on particle transport must be considered. Brownian motion results from random variations in the force exerted on the particle by background-gas molecular bombardment, and gives rise to particle diffusion along concentration gradients. Also, Brownian motion implies that particle trajectories are no longer deterministic; that is, identical particles started at the same initial location with the same initial conditions will not follow the same path through the reactor. In this case, an Eulerian formulation of particle transport is used, in which the particles are treated as a continuum or cloud and the particle concentration field is calculated (inertia is neglected). Particle deposition is determined in terms of a particle flux at the wafer's surface, $J_{o}\left(\# / \mathrm{cm}^{2} / \mathrm{s}\right)$, which is calculated from the surface concentration gradient (where particle interception is neglected):

$$
J_{o}=\left.\mathcal{D} \frac{d n}{d z}\right|_{z=0}
$$

where the $n$ is the particle concentration and $\mathcal{D}$ is the particle diffusion coefficient. Note that a general expression for particle flux would include both a diffusional term, given by Equation (4.4), and a drift-velocity term, given by $n \tilde{V}_{p}^{t}$. In Equation (4.4) only the diffusional term is shown because, under our assumption that particle concentration vanishes at surfaces, the drift-velocity contribution must also vanish at the wafer. Thus, even when external forces are controlling deposition, a thin boundary layer must exist near the wafer wherein the concentration drops from the free-stream value to zero at the wafer's surface. The particle collection efficiency is then calculated as the ratio of particle flux to the wafer divided by the particle source term (number of particles being released from the trap). More detailed discussion is provided in the next chapter.

Equation (4.4) can be extended to account for particle interception by evaluating the concentration derivative at $z=r_{p}$ (instead of zero):

$$
J_{o}=\left.\mathcal{D} \frac{d n}{d z}\right|_{z=r_{p}}
$$




\subsubsection{External Force Limit}

As in the previous section, an analytic result can be derived for deposition efficiency in the limiting case where external forces control particle deposition (particle inertia, interception, and diffusion are all neglected):

$$
\eta=\left(\frac{R_{c r i t}}{R_{W}}\right)^{2}=\left(\frac{R_{i}}{R_{f}}\right)^{2}=\frac{\left|V_{p}^{t}\right|}{\left|V_{p}^{t}\right|+|u(h)|} \quad V_{p}^{t} \leq 0
$$

which is the same as Equation (4.2) except that the gas axial velocity at the trap location replaces the mean gas velocity in the denominator. For net drift velocities greater than zero (net external force pushing particles away from the wafer), no particle deposition on the wafer is predicted. Equation (4.6) provides a lower bound for particle deposition, as inertial and diffusional effects can only increase deposition from what is predicted. As expected, the collection efficiency tends toward unity as the particle trap moves closer to the lower plate $(h \rightarrow 0)$ because the axial gas velocity must approach zero at the plate surface. For particles which ultimately deposit on the wafer, Equation (4.6) also can be used to determine the radial position on the wafer at which a particle is collected $-R_{f}$ based on its starting position $r=R_{i}$ and $z=h$. As discussed in the previous section, particles which deposit on the wafer are those which start nearest to the reactor centerline. It should be noted that both the Eulerian and Lagrangian collection efficiencies defined above must tend to Equation (4.6) in the limit when particle diffusion, inertia, and interception effects are all negligible.

Equation (4.6) can be extended to include particle interception as in the previous section:

$$
\eta=\left(\frac{R_{c r i t}}{R_{W}}\right)^{2}=\left(\frac{R_{i}}{R_{f}}\right)^{2}=\frac{\left|V_{p}^{t}\right|+\left|u\left(r_{p}\right)\right|}{\left|V_{p}^{t}\right|+|u(h)|} \quad V_{p}^{t} \leq 0
$$

As before, particle collection is now predicted in the absence of (or for weak) external forces, and is seen to depend on the flowfield through flow velocity terms in both the numerator and denominator. Both the Eulerian and Lagrangian collection efficiencies defined above must tend to Equation (4.7) in the limit where particle diffusion and inertial effects are negligible. 


\section{Diffusion-Enhanced Deposition}

Because of semiconductor manufacturing trends towards smaller feature sizes on integrated circuits and low-pressure processing, the size of killer defects are now small enough so that the role of particle Brownian motion in particle transport and deposition must be considered in addition to deposition from external forces (Cooper, 1986; Cooper et al., 1989). This chapter builds on the analysis of the previous chapter, here considering particle deposition due to the simultaneous action of convective diffusion and uniform external forces (see also Rader et al., 1999). As before, a showerhead-type reactor is considered and the particles are assumed to start in planar traps between the plates. Physical processes leading to this initial condition would include particles generated in situ by nucleation, and particles that are originally trapped between the plates during a plasma process, which are subsequently released at plasma extinction (Choi et al., 1996).

When a radio frequency $(\mathrm{RF})$ plasma is present, contaminant particles in it generally accumulate near the bulk plasma-sheath boundary of the discharge (Roth et al., 1985). Sommerer et al. (1991) and Barnes et al. (1992) proposed that particle transport in the discharge is dominated by two forces: electrostatic and viscous ion drag. The electrostatic force accelerates negatively charged particles towards the center of electropositive plasmas, while viscous ion drag accelerates particles in the direction of net ion flux (generally towards plasma boundaries). Particle "traps" occur in regions where the sum of forces acting on the particle vanishes. In many cases these traps are approximately planar and parallel to the plates (Choi et al., 1994); a schematic of a planar trap in a parallel-plate reactor is shown in Figure 12, where the particles are uniformly distributed at a distance $h$ above the lower plate. Only 1D planar traps are considered in this work, although a variety of other trap structures (rings, domes, etc.) are known in the literature. For any trap structure more complicated than an infinite plane the problem becomes inherently $2-\mathrm{D}$, which is beyond the scope of the present analysis.

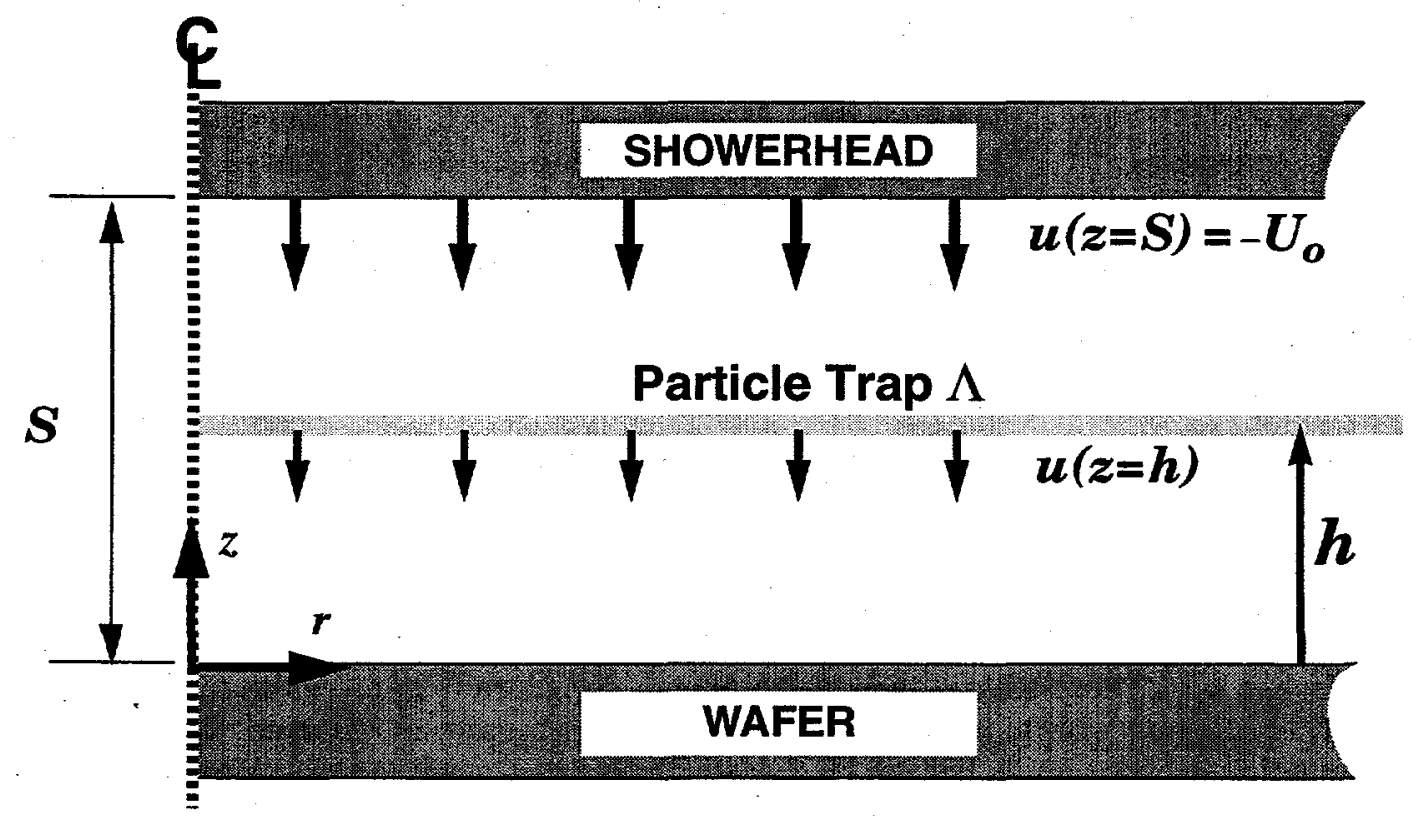

Figure 12. Geometry. Diagram of parallel, infinite-plate geometry with particles in a planar trap located a distance $h$ from the lower plate. 
At the end of the plasma process step, the discharge is extinguished and the plasma-induced forces responsible for particle trapping are assumed to dissipate rapidly (compared to particle transport times) in the afterglow. In this work, we assume that the charged particles are rapidly neutralized after the plasma extinction and can therefore be treated as neutral particles as experimentally observed by Jellum et al. (1991), Shiratani et al. (1991) and Yeon (1995) (although a recent study by Collins et al. (1996) suggests that some particles might retain a few residual charges in the afterglow). Under the assumption of rapid neutralization, the particles are released from the traps and can deposit on the wafer as a result of gravity, thermophoresis, inertia, or Brownian motion (diffusion).

A planar particle source could also be encountered for particles generated by nucleation. As the time scale for particle nucleation and growth can be quite small compared to transport time scales, it is possible to model the nucleated particles as appearing suddenly at a location between the plates, at which point the analysis of their transport is analogous to particles released from a plasma trap. The analogy is completed by considering that, for 1-D parallel plate flow, the region of particle nucleation would be expected to be planar. One difference between these two particle-source scenarios is that the source term resulting from nucleation would be continuous, while the source term for a plasma-trap release would be a transient event characterized by the number of particles in the trap at the time of release.

In this chapter, particle inertia is neglected and the Eulerian approach of Section 2.6 is used to simulate particle transport resulting from the combination of particle external forces and Brownian motion. The key result of the present analysis is the particle collection efficiency - which is the fraction of trapped particles which end up on the wafer. For creeping flow between infinite parallel plates, and with a few additional assumptions, an analytic expression is derived that gives the particle collection efficiency as a function of four dimensionless parameters: $\operatorname{Re}, \tilde{h}, \tilde{V}_{p}^{t}$, and Pe (a fifth - the interception parameter $d_{p} / S$ - is neglected in this chapter). The first parameter, the Reynolds number, completely specifies the flow field for the infinite parallel-plate geometry - as demonstrated in the analytic low-Re approximation to the flow field given in Equation (3.5). The second parameter, the dimensionless trap height, $\tilde{h}=h / S$, specifies the position of the particle source term. The influence of external forces enters through the third parameter, the dimensionless particle drift velocity, $\tilde{V}_{p}^{t}=V_{p}^{t} / U_{o}$, which is defined as the $z$-component of the net drift velocity. The fourth parameter is the particle Peclet number, $\mathrm{Pe}=S U_{o} / \mathcal{D}$, which is a measure of the relative importance of particle Brownian motion. In the free molecular limit the Peclet number is proportional to diameter squared, so that $\mathrm{Pe}^{1 / 2}$ can be thought of as a dimensionless particle size. A theoretical analysis of particle deposition from simultaneous diffusion and external forces is presented in Section 5.1. The effect of the various dimensionless parameters on particle capture efficiency is explored in Section 5.2, and Section 5.3 presents several examples in dimensional terms.

The present analysis closely follows the previous work of Peters et al. (1989) who investigated the convective-diffusive deposition of particles onto disks in semi-infinite stagnation-point flow (such as for a wafer exposed to the downward flow in a clean room). Although the present creeping, stagnation-point solution for flow between parallel plates is closely related to that used by Peters et al., there are some important differences. For example, the earlier work relies on a boundary layer approximation which is only valid near the wafer, while the present analysis is valid everywhere between the two plates. The finite domain of the present geometry also distinguishes it from the semi-infinite domain of Peters. In the earlier work there exists some ambiguity as to where to enforce the far-field velocity condition (Broday et al., 1997) while in the present case this ambiguity is removed. The two studies also differ in the particle concentration boundary conditions that are used: in the earlier work particle concentration was assumed to be zero at the disk and to approach a constant infinitely far away from it, while in this work the two plates are considered perfectly absorbing with a planar particle source located between them. Consequently, the present results for particle deposition show significant differences compared to the work of Peters et al. - particularly in the diffusive limit. 


\subsection{Theory}

\subsubsection{Problem Definition}

In this section, we assume the geometry shown in Figure 12: axisymmetric flow between two infinite, parallel plates (a showerhead and a wafer) separated by a distance $S$. The effect of jetting out of the showerhead holes is neglected, so that the flow is assumed to be uniformly distributed across the bottom of the showerhead with velocity $-U_{o}$. The flow is assumed to be isothermal (constant gas properties), steady, laminar, incompressible, and viscous such that the quasi-one-dimensional analytic result of Chapter 3 - Equation (3.5) - can be used. For ease of reference, that result is also given here:

$$
\begin{gathered}
\tilde{u}(\tilde{z})=\frac{u(z)}{U_{o}}=2 \tilde{z}^{3}-3 \tilde{z}^{2}+\frac{\operatorname{Re}}{70}\left(2 \tilde{z}^{7}-7 \tilde{z}^{6}+18 \tilde{z}^{3}-13 \tilde{z}^{2}\right) \\
\tilde{v}(\tilde{r}, \tilde{z})=\frac{v(r, z)}{U_{o}}=\tilde{r}\left[3 \tilde{z}-3 \tilde{z}^{2}-\frac{\operatorname{Re}}{70}\left(7 \tilde{z}^{6}-21 \tilde{z}^{5}+27 \tilde{z}^{2}-13 \tilde{z}\right)\right]
\end{gathered}
$$

As discussed in Chapter 3, Equation (5.1), is reasonably accurate for flow Reynolds numbers less than about four based on comparison to more accurate numerical finite-element simulations.

The particles are assumed to enter the domain at a steady generation rate $\Lambda_{h}\left(\# / \mathrm{cm}^{2} / \mathrm{sec}\right)$ from a planar source located a distance $h$ from the wafer. Although this description adequately applies to a continuous source such as particle nucleation, it is not immediately obvious that a steady-state analysis is applicable to the transient case where a finite number of particles are simultaneously released from a trap at time $t=0$. Analysis of the governing equations reveals that the particle collection efficiencies from the steady-state and transient problems are in fact the same under the following conditions: 1) steady flow field, 2) infinite parallel-plate (1-D) domain, 3) radially uniform distribution of initial particle positions for the transient problem, and 4) radially uniform particle source for the steadystate problem. To confirm this contention, particle transport calculations using the present steadystate Eulerian approach have been compared with the Brownian dynamics simulations (BDS) of Choi et al. (1996). Choi and coworkers solved the Langevin equation directly using a massively parallel numerical Lagrangian particle tracking model which included a fluctuating Brownian force; transport calculations were presented for particles that were initially distributed in planar traps in a parallelplate geometry similar to that of interest here. The BDS method is inherently transient in nature, in that a large number of particles were initially distributed uniformly throughout the trap, and their trajectories followed in time until the particles either deposited on a plate or left the calculation domain. For comparison with the present approach, Brownian dynamics simulations were performed with the analytic velocity field given by Equation (5.1). As expected, BDS results for particle collection efficiency were in excellent agreement with the steady-state Eulerian formulation presented here. Thus, the analytic result for particle collection efficiency given below applies equally well for a steadystate particle planar source as for the case of a cloud of particles released from a planar trap.

\subsubsection{Solution of the Eulerian Particle Transport Equation}

Neglecting particle inertia, the Eulerian expression for particle concentration, $n\left(\# / \mathrm{cm}^{3}\right)$, was given in Chapter 2 by Equation (2.33) which is reproduced here:

$$
\frac{\partial n}{\partial t}+\boldsymbol{U} \cdot \nabla n=\nabla \cdot \mathcal{D} \nabla n-\nabla \cdot\left(\boldsymbol{V}_{p}^{t} n\right)+\Lambda
$$

where $\mathcal{D}\left(\mathrm{cm}^{2} / \mathrm{sec}\right)$ is the Stokes-Einstein particle diffusion coefficient, $\Lambda\left(\# / \mathrm{cm}^{3} / \mathrm{sec}\right)$ is the particle source term, and $V_{p}^{t}$ is the net drift velocity vector. Consistent with our flow assumptions, the concentration field is assumed to be steady and one-dimensional (depending only axial position). Also, for 
isothermal flow, the diffusion coefficient and particle drift velocity are constant. With these assumptions and simplifications, Equation (5.2) may be rewritten as:

$$
u \frac{d n}{d z}=\mathcal{D} \frac{d^{2} n}{d z^{2}}-V_{p}^{t} \frac{d n}{d z}+\Lambda
$$

In Equation (5.3), $V_{p}^{t}$ is the $z$-component of the net drift velocity vector and $u$ is the axial velocity field given by Equation (5.1). For boundary conditions, the assumption of perfectly absorbing walls is made which implies that the particle concentration is zero at both the upper and lower plates, i.e. $n(z=0)=n(z=S)=0 .{ }^{1}$ Note that by assuming an absorbing surface at the showerhead we are neglecting the showerhead holes, but this is reasonable as the holes typically account for only a few per cent of the total showerhead surface.

For this analysis, the particle source is assumed to be infinitely thin so that:

$$
\Lambda=\Lambda_{h} \delta_{d}(z-h)
$$

where $\Lambda_{h}\left(\# / \mathrm{cm}^{2} / \mathrm{sec}\right)$ is a constant area source term and $\delta_{d}\left(\mathrm{~cm}^{-1}\right)$ is the Dirac delta function. Although the following derivation also could be followed for a finite-thickness source term, the resulting analytic expression for particle collection efficiency would be much more complicated than that given below.

To simplify later discussions, it is convenient to nondimensionalize Equation (5.3). The appropriate characteristic length and velocity scales are $S$ and $U_{o}$, respectively. A characteristic particle concentration, $n_{o}$, can be defined based on the particle source strength and gas inlet velocity:

$$
n_{o}=\frac{\Lambda_{h}}{U_{o}}
$$

Using these definitions, Equation (5.3) may be nondimensionalized as:

$$
\tilde{u} \frac{d \tilde{n}}{d \tilde{z}}=\frac{1}{\operatorname{Pe}} \frac{d^{2} \tilde{n}}{d \tilde{z}^{2}}-\tilde{V}_{p}^{t} \frac{d \tilde{n}}{d \tilde{z}}+\delta_{d}(\tilde{z}-\tilde{h})
$$

where $\tilde{n}=n / n_{o}, \tilde{u}=u / U_{o}, \tilde{z}=z / S, \tilde{V}_{p}^{t}=V_{p}^{t} / U_{o}, \mathrm{Pe}=S U_{o} / \mathcal{D}$, and $\tilde{h}=h / S$. As discussed above, the solution for the dimensionless concentration is completely determined by the dimensionless groups $\mathrm{Pe}, \tilde{h}$, $\tilde{V}_{p}^{t}$, and $\operatorname{Re}$ (which enters implicitly as $\tilde{u}$ depends on Reynolds number).

After defining a dimensionless concentration gradient

$$
\tilde{G}=\frac{d \tilde{n}}{d \tilde{z}}
$$

Equation (5.6) can be rewritten as:

$$
\frac{d \tilde{G}}{d \tilde{z}}-\operatorname{Pe}\left(\tilde{u}+\tilde{V}_{p}^{t}\right) \tilde{G}=-\operatorname{Pe} \delta_{d}(\tilde{z}-\tilde{h})
$$

\footnotetext{
1. Deposition by interception, due to the finite size of the particle, is neglected. To account for interception requires that the boundary conditions be given as $n\left(z=r_{p}\right)=n\left(z=S-r_{p}\right)=0$ where $r_{p}$ is the particle radius.
} 
The solution to Equation (5.8) is:

$$
\tilde{G}=\operatorname{Sh} \cdot \exp (A)-\exp (A) \int_{0}^{\tilde{z}} \operatorname{Pe} \delta_{d}(\tilde{z}-\tilde{h}) \exp (-A)(d \tilde{z})
$$

where:

$$
A(\tilde{z})=\operatorname{Pe}\left[\frac{1}{2} \tilde{z}^{4}-\tilde{z}^{3}+\frac{\operatorname{Re}}{70}\left(\frac{1}{4} \tilde{z}^{8}-\tilde{z}^{7}+\frac{9}{2} \tilde{z}^{4}-\frac{13}{3} \tilde{z}^{3}\right)+\tilde{V}_{p}^{t} \tilde{z}\right]
$$

and the integration constant Sh is the dimensionless concentration gradient at the lower plate, frequently referred to as the Sherwood number (e.g., Peters et al., 1989). To determine Sh, apply the boundary conditions $\tilde{n}(\tilde{z}=0)=\tilde{n}(\tilde{z}=1)=0$ after integrating Equation (5.7):

$$
\int_{0}^{1} \frac{d \tilde{n}}{d \tilde{z}} d \tilde{z}=\int_{0}^{0} d \tilde{n}=0=\int_{0}^{1} \tilde{G} d \tilde{z}
$$

Solving Equation (5.11) for Sh gives:

$$
\mathrm{Sh}=\frac{\operatorname{Pe} \cdot \int_{\tilde{h}}^{1} \exp \left\{\operatorname{Pe}\left[\frac{1}{2}\left(t^{4}-\tilde{h}^{4}\right)-\left(t^{3}-\tilde{h}^{3}\right)+\frac{\operatorname{Re}}{70}\left(\frac{1}{4}\left(t^{8}-\tilde{h}^{8}\right)-\left(t^{7}-\tilde{h}^{7}\right)+\frac{9}{2}\left(t^{4}-\tilde{h}^{4}\right)-\frac{13}{3}\left(t^{3}-\tilde{h}^{3}\right)\right)+\tilde{V}_{p}^{t}(t-\tilde{h})\right]\right\} d t}{\int_{0}^{1} \exp \left\{\operatorname{Pe}\left[\frac{1}{2} t^{4}-t^{3}+\frac{\operatorname{Re}}{70}\left(\frac{1}{4} t^{8}-t^{7}+\frac{9}{2} t^{4}-\frac{13}{3} t^{3}\right)+\tilde{V}_{p}^{t} t\right]\right\} d t}
$$

A similar integral expression for particle deposition in a semi-infinite, axisymmetric stagnation-point flow hase been reported previously by Peters et al. (1989, see Equation 18).

\subsubsection{Particle Collection Efficiency}

The particle collection efficiency is found as the ratio of particle flux to the lower plate divided by the total number of particles entering the reactor:

$$
\eta=\frac{\left.A_{W} \mathcal{D} \frac{d n}{d z}\right|_{z=0}}{A_{W} \int_{0}^{S} \Lambda_{h} \delta(z-h) d z}=\frac{\frac{\mathcal{D} \Lambda_{h} d \tilde{n}}{S U_{o}} \frac{d \tilde{z}}{\tilde{z}=0}}{\Lambda_{h}}=\frac{S h}{\mathrm{Pe}}
$$

where $A_{W}$ is the wafer (and showerhead) area in the $r-\theta$ plane. Thus, an analytic result for the particle collection efficiency is given by Equations (5.12) and (5.13) - although the solution requires numerical quadrature. The dependence of the collection efficiency on the four dimensionless groups, $\operatorname{Re}, \tilde{h}, \tilde{V}_{p}^{t}$, and $\mathrm{Pe}$, is clearly shown in Equations (5.12) and (5.13). The use of collection efficiency as the dimensionless number characterizing the deposition process is preferred here over the Sherwood number primarily for two reasons: 1) determination of the collection efficiency is also straightforward for Lagrangian formulations (such as applied to showerhead-enhanced inertial deposition in Chapter 6) in which critical trajectories can be calculated, and 2) efficiency is a commonly accepted concept within the semiconductor industry (e.g., yield). In practical terms, an efficiency of unity indicates that all particles in the chamber are depositing on the wafer, while an efficiency of zero indicates none are. 


\subsubsection{Particle Flux}

The particle deposition rate on the wafer is found as the product of collection efficiency times the number of particles released from the trap (or generated by nucleation):

$$
J_{o}=\mathcal{D} \frac{d n}{d z}=\left.\frac{\mathcal{D} \Lambda_{h}}{S U_{o}} \frac{d \tilde{n}}{d \tilde{z}}\right|_{\tilde{z}=0}=\Lambda_{h} \frac{S h}{\mathrm{Pe}}=\Lambda_{h} \eta
$$

When the nature of the source term $\Lambda_{h}$ is not known, the best strategy for reducing the number of defects on a wafer is to choose conditions that inhibit particle transport to the wafer, i.e., minimize the collection efficiency. One potential weakness of this strategy is if process conditions selected to reduce the collection efficiency result in a corresponding increase in the particle generation rate; this possibility is certainly of concern for particle nucleation.

\subsubsection{Theoretical Asymptotic Limits}

To explore the behavior of Equations (5.12) and (5.13), we consider several asymptotic limits for which the collection efficiency can be found.

Trap-height limits.

As the trap approaches the lower plate, $\tilde{h} \rightarrow 0$, the integrals of Equation (5.12) become equal and the collection efficiency tends to unity; as expected, in this limit all of the particles deposit on the lower plate. On the other hand, when $h \rightarrow 1$, the integral in the numerator of Equation (5.12) tends to zero and the efficiency tends to zero; in this limit all of the particles deposit on the upper plate. Thus, according to the present model, particles that originate at the showerhead never reach the wafer since the showerhead is treated as a perfectly absorbing surface. Actually, the present model breaks down in the region immediately below the showerhead, as the flow consists of many individual jets which emerge from holes in the showerhead and remain distinct for some distance before merging into a uniform flow. Particles passing through the showerhead holes might actually penetrate into the reactor due to inertial effects, or by being swept along by the relatively high gas velocities in the jet.

Diffusion dominated limits.

For small particles, Brownian motion becomes increasingly important, the Peclet number becomes small, and the exponential functions within the integrals of Equation (5.12) tend towards unity. For $\mathrm{Pe}<<1$, the exponential functions of Equation (5.12) can be approximated by a Taylor series and the leading terms integrated analytically to give:

$$
\eta \rightarrow 1-\tilde{h} \quad \text { as } \quad \mathrm{Pe} \rightarrow 0 \quad \text { for all } \tilde{V}_{p}^{t}
$$

Thus, when diffusion dominates, the fraction of particles reaching the lower plate is linearly related to the distance between it and the trap. In this limit, particle concentration profiles are linear in the regions above and below the source, and each slope is determined by the proximity of the trap to the appropriate surface. The efficiency limit of Equation (5.15) can also be derived independently through an analysis of pure diffusional transport (neglecting convection and external forces) with a delta-function source term. The limit of Equation (5.15) applies for any external forces and trap position, although the rate of approach to the limit with Pe will depend on the specific values of $\tilde{V}_{p}^{t}$ and $\tilde{h}$.

In the absence of external forces $\left(\tilde{V}_{p}^{t}=0\right)$, deposition must be controlled by diffusion for all Peclet numbers. With the additional constraint that the particle size (and Peclet number) is very large, an analytic approximation may be obtained using Laplace's method (Bender and Orszag, 1978) to ap- 
proximate the integrals in both the denominator and numerator of Equation (5.12). In Laplace's method, the arguments of the exponentials are expanded in a Taylor series about their maxima within the integration limits (which occur at the lower-limits of integration here) and the resulting series can then be integrated analytically to find:

$$
\eta \rightarrow \frac{\operatorname{Pe}^{-2 / 3}\left(1+\frac{13 R e}{210}\right)^{1 / 3}}{\Gamma\left(\frac{4}{3}\right)|\tilde{u}(\tilde{h})|} \quad \text { as } \quad \mathrm{Pe} \rightarrow \infty \quad \text { for } \tilde{V}_{p}^{t}=0
$$

where $\Gamma(4 / 3)=0.89298$ is the Gamma function. Peters et al. (1989) arrived at a similar result in their analysis for infinite stagnation point flow; their result for Sherwood number can be converted to efficiency to give $\eta=0.616 \mathrm{Pe}^{-2 / 3}$. Both results show the same Peclet dependence, and in both cases the coefficient is of order one. The present result, however, shows a specific dependence on both the flow Reynolds number and the trap position (note that the Reynolds dependence enters explicitly in the numerator and implicitly through the local fluid velocity in the denominator). The approximations underlying Equation (5.16) break-down when the trap is near a plate - particularly near the wafer in which case the denominator tends to zero. Based on numerical integrations of Equation (5.12), the limiting result of Equation (5.16) is accurate to within $10 \%$ for $0.2<\widehat{h}<0.995$ and Pe>10 $0^{3}$.

\section{Attractive external-force dominated limit.}

In the limit where particle size and Peclet number become large, the contribution of Brownian motion to particle transport becomes negligible compared to any attractive external forces $\left(\tilde{V}_{p}^{t}<0\right)$. In this case (having already neglected inertia and interception), the efficiency must tend to the limit given in Chapter 4 - Equation (4.6) - for the case where external forces control particle deposition:

$$
\eta \rightarrow \frac{\left|\tilde{V}_{p}^{t}\right|}{\left|\tilde{V}_{p}^{t}\right|+|\tilde{u}(\tilde{z}=\tilde{h})|} \quad \text { as } \quad \mathrm{Pe} \rightarrow \infty \quad \text { for } \tilde{V}_{p}^{t}<0
$$

To obtain this limit from Equation (5.12), the integrals in both the denominator and numerator can be evaluated using Laplace's method as discussed above: the numerator of Equation (5.12) is found to be $-1 /\left[\mathrm{Pe}\left(\tilde{u}(\tilde{h})-\tilde{V}_{p}^{t}\right)\right]$, while the denominator is $-1 /\left(\mathrm{Pe} \tilde{V}_{p}^{t}\right)$. Substituting these two expressions into Equation (5.12) and using Equation (5.13) gives the correct external force limit of Equation (5.17). Note that Equations (5.16) and (5.17) might appear inconsistent in the limit where $\tilde{V}_{p}^{t}$ approaches zero; in fact, the limit of Equation (5.17) holds for even very small drift velocities although the Pe number at which the limit is achieved becomes increasingly larger. The root cause of any apparent inconsistencies is the attempt to isolate either diffusive or external-force driven deposition; the true collection efficiency is a combination of the two effects as represented in the complete solution of Equations (5.12) and (5.13). Cooper et al. (1989) have shown that a linear addition of the independent terms for external-force and diffusion-driven deposition - here Equations (5.16) and (5.17) - adequately approximates the combined deposition rate for infinite axisymmetric stagnation point flow. This approximation is explored below for the present geometry.

\section{Repulsive external-force dominated limit.}

An external force directed away from the wafer $\left(\tilde{V}_{p}^{t}>0\right)$ inhibits particle deposition on the wafer. In the absence of diffusion, a repulsive force creates a particle-free zone between the wafer and $\delta$ (the socalled "turning point"), which is the dimensionless axial position at which the upward external and downward drag forces are equal. ${ }^{2}$ In this case, the only way for a particle to deposit on the wafer is

\footnotetext{
2. This position is defined by $\tilde{V}_{p}^{t}=-\tilde{u}(\delta)$ and is independent of the initial trap position, $h$. Note that $0<\delta<1$.
} 
when the random fluctuations in position associated with Brownian motion become large enough to overcome the resisting energy barrier. For example, in the small Peclet-number limit, diffusion overwhelms the repulsive force and the deposition efficiency is given by Equation (5.15). An interesting limit for the repulsive-force case is that of large Peclet numbers, for which the effects of Brownian motion are just becoming noticeable. For large Pe, Laplace's method again can be used to evaluate the integrals of Equation (5.12). Although there are several specific cases, the general form of the result is $\eta \sim \alpha \exp (-\phi \mathrm{Pe}) \quad$ where $\alpha$ and $\phi$ are positive functions, $\alpha\left(\mathrm{Pe}^{1 / 2}, \delta\right)$ and $\phi\left(\delta, \tilde{h}, \tilde{V}_{p}^{t}\right)$, and where $\delta$ is the dimensionless turning point described above. In all cases, as diffusion vanishes (Pe becomes large) the exponential term dominates and efficiency exponentially tends to zero. In the presence of a repulsive force, the particle concentration field for large $P e$ is of the boundary-layer type, where the concentration decreases rapidly from the its value at the trap or turning point (whichever is nearest the wafer) as you move towards the wafer. For decreasing Pe, the thickness of this boundary layer increases until, in the diffusive limit, the concentration profile becomes linear between $\delta$ and the wafer.

One case requires special attention, which is that where a strong-enough repulsive force is acting so that the net drift velocity is upward and larger than the flow velocity at the trap position: $\tilde{V}_{p}^{t}>|\tilde{u}(\tilde{h})|$. In this case, the turning point is above the particle starting position, so that the particles move upward upon release. In this case, the value of the integral in the numerator of Equation (5.12) becomes very large with increasing Pe and numerical quadrature can become troublesome. ${ }^{3}$ The approach used here is to monitor the numerical value of the integral until the limit of machine accuracy is reached, and then to replace Equations (5.12) and (5.13) with an analytic asymptotic value found by application of Laplace's method:

$$
\eta \rightarrow \exp \left\{-\operatorname{Pe}\left[\frac{\tilde{h}^{4}}{2}-\tilde{h}^{3}+\frac{\operatorname{Re}}{70}\left(\frac{\tilde{h}^{8}}{4}-\tilde{h}^{7}+\frac{9}{2} \tilde{h}^{4}-\frac{13}{3} \tilde{h}^{3}\right)+\tilde{V}_{p}^{t} \tilde{h}\right]\right\} \mathrm{Pe} \rightarrow \infty \text { for } \tilde{V}_{p}^{t}>|\tilde{u}(\tilde{h})|
$$

As expected for the case of a strong repulsive force, the efficiency given by Equation (5.18) tends towards zero in the large Peclet limit where diffusion becomes negligible.

\subsection{Nondimensional Results}

This section presents calculations of particle collection efficiency using numerical quadrature of Equations (5.12) and (5.13) using a fourth-order Runge-Kutta technique with automatic error control. Both local and global error control parameters can be set, and convergence tests showed that the resulting integrals were unchanged in the fifth place when these parameters were set to $10^{-10}$. As discussed above, efficiency is found to be a function of four dimensionless parameters: Re, $\tilde{h}, \dot{V}_{p}^{t}$, and Pe (a fifth - the interception parameter $d_{p} / S$ - is neglected in this work). Calculations of particle collection efficiency versus Peclet number are shown in Figure 13 for creeping flow $(\operatorname{Re}=0)$ for three attractive forces (characterized by $\tilde{V}_{p}^{t}=-0.1,-0.5$, and -1.0 ), for no external force $\left(\tilde{V}_{p}^{t}=0\right.$ ), and for three repulsive forces $\left(\tilde{V}_{p}^{t}=0.1,0.5\right.$, and 1.0). Plots are shown for three different trap heights, where the particles are trapped: a) near the wafer $(h / S=0.1)$, b) midway between the wafer and showerhead $(h / S=0.5)$, and c) near the showerhead $(h / S=0.9)$. To verify that the quadrature method is performing properly, the numerical results are first compared to the asymptotic limits discussed in the previous section; next follows a general discussion of the effects of the controlling parameters on collection efficiency. Finally, this section concludes with a discussion of the effectiveness of approximating deposition by a linear combination of terms from the various deposition mechanisms.

3. The denominator of Equation (5.12) tends to infinity faster than the numerator, however, so that the ratio of the two integrals tends toward zero. However, significant computational errors are introduced by taking the ratio of two very large numbers. 
a)

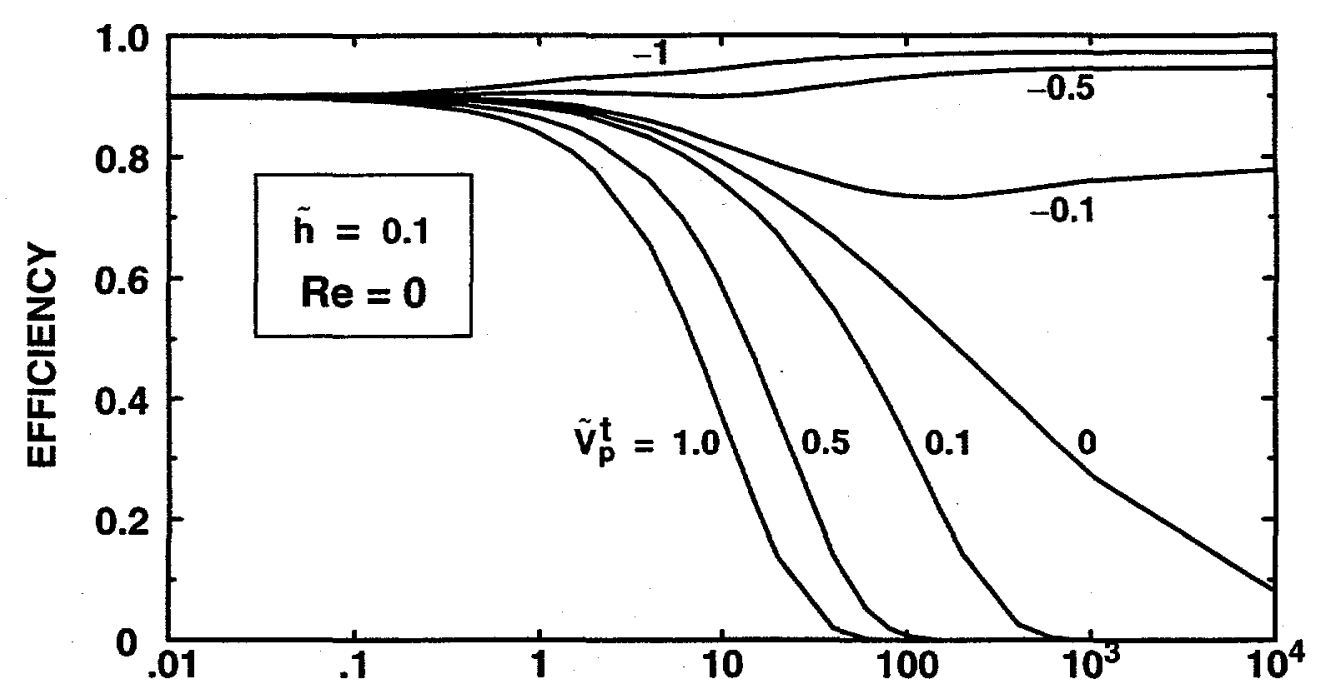

b)

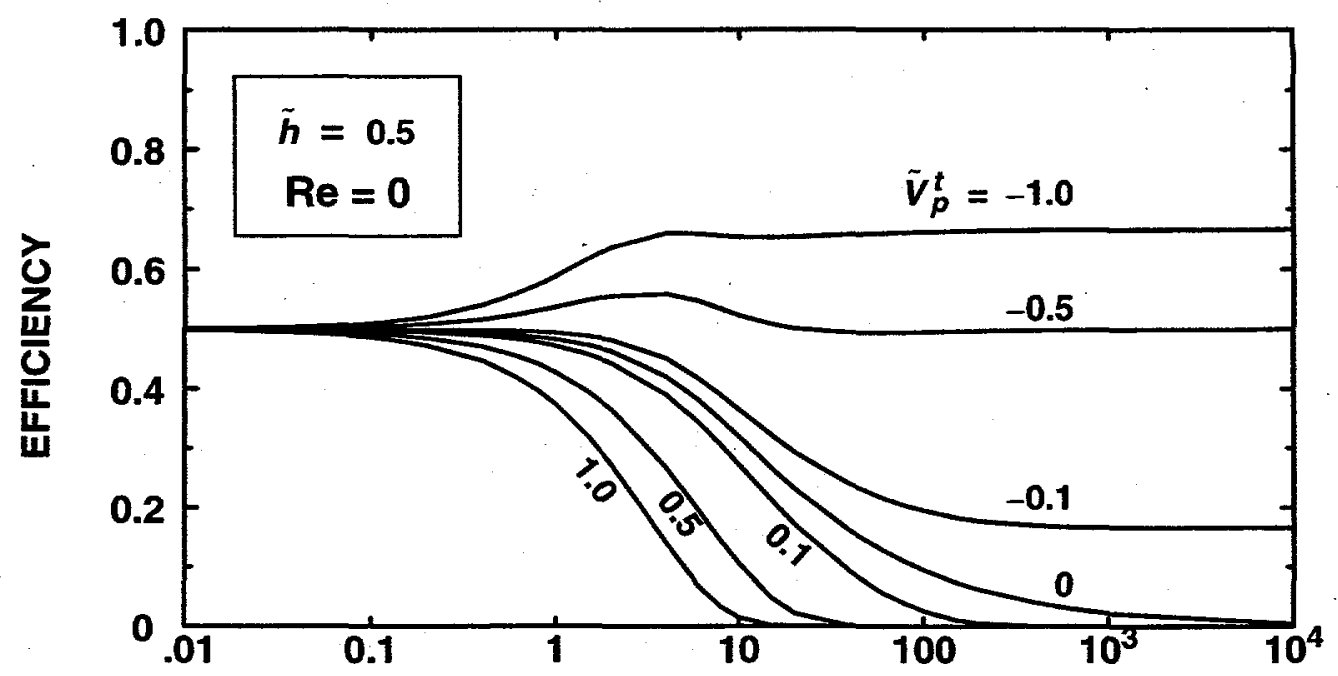

c)

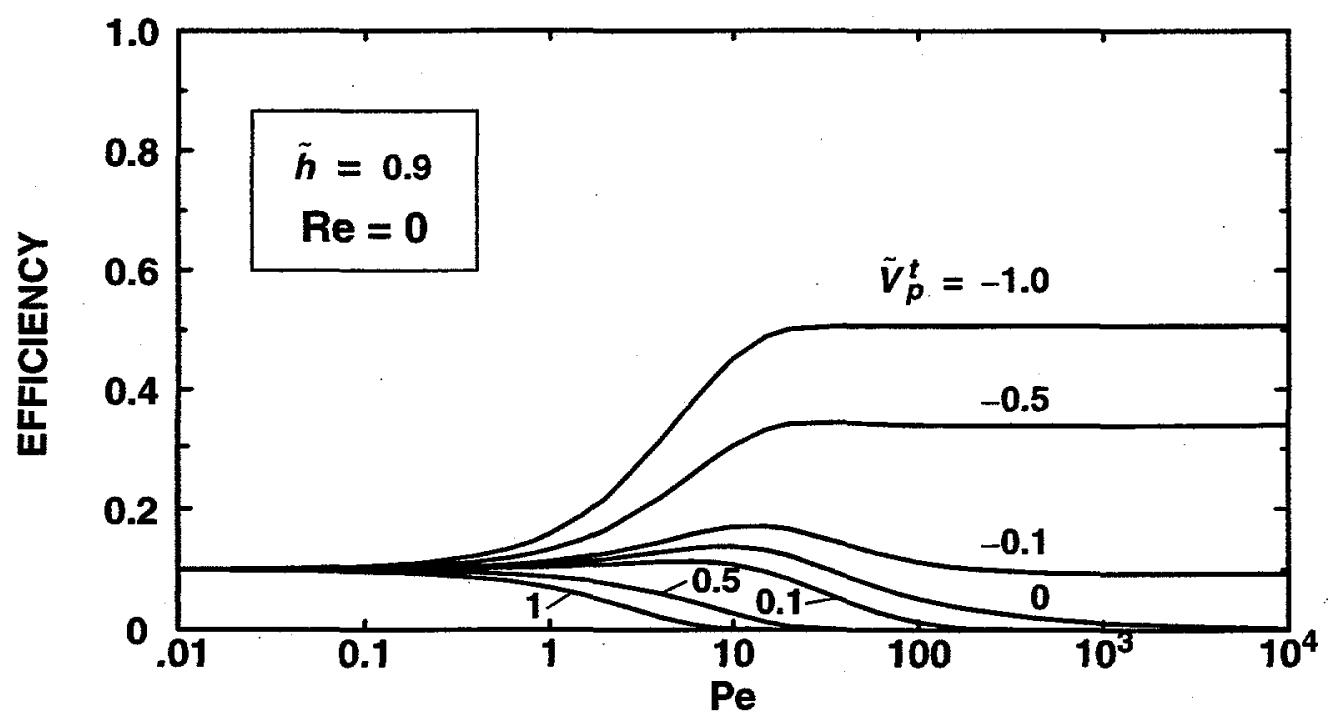

Figure 13. Efficiency vs. Pe for Various Drift Velocities. Figures show calculated efficiencies for particles starting in traps at: a) $h / S=0.1$, b) $h / S=0.5$, and c) $h / S=0.9$. 


\subsubsection{Comparison of Calculated Results to Asymptotic Limits}

First, for rapidly diffusing particles (small particles and small $\mathrm{Pe}$ ), the results of Figure 13 show that $\eta \rightarrow 1-\tilde{h}$ for any external force (i.e., for all $\tilde{V}_{p}^{t}$ ) as required by Equation (5.15). For example, a highly diffusive particle starting at a trap midway between the plates is equally likely to be collected on the showerhead as on the wafer, so that the efficiency tends to 0.5 . This limit is intuitively pleasing in that particles traps near the wafer give proportionately higher collection efficiencies that particle traps near the showerhead. It is seen in Figure 13 that the limit of Equation (5.15) is essentially reached in all the cases shown for $\mathrm{Pe}<0.1$. Thus, under the present assumptions, particle deposition is dominated by diffusion for $\mathrm{Pe}<0.1$.

Diffusion must also control deposition in the absence of any external force $\left(\tilde{V}_{p}^{t}=0\right)$; the large Pecletnumber limit of this case is given by Equation (5.16). The range of Peclet numbers over which Equation (5.16) is approached, however, is found to depend strongly on trapping height, particularly for the case where the particles are initially trapped near the wafer. For example, for $\tilde{h}=0.1$, the numerical integration results approach Equation (5.16) to within $25 \%$ only for $\mathrm{Pe}>10,000$. The same accuracy is achieved for $\mathrm{Pe}>20$ for trapping heights in the range $0.7<\tilde{h}<0.9$, and for $\mathrm{Pe}>400$ for 0.2 $<\tilde{h}<0.99$. In any case, the asymptotic limit is achieved (as required) at sufficiently high Peclet numbers. In the absence of external forces, the small-Pe limit of Equation (5.15) must still apply, and it can be seen that the $\tilde{V}_{p}^{t}=0$ curves all approach the proper limit for $\mathrm{Pe}<0.1$.

In the large particle or high-Pe limit, diffusional effects vanish and the collection efficiency must approach the velocity-ratio limit given by Equation (5.17) for the case of an attractive external force $\left(\tilde{V}_{p}^{t}<0\right)$. For example, for particles starting at a trap midway between the plates with a dimensionless drift velocity of -0.5 , the large-Pe collection efficiency must tend towards one-half (from Equation (5.17) and using the fact that the dimensionless gas velocity at the midplane is $\tilde{u}(h / S=0.5)=-0.5)$. As seen in Figure 13, the non-diffusing deposition limit of Equation (5.17) is essentially reached for Peclet numbers larger than $-10^{2}$ or $-10^{3}$ depending on the strength of the external force and the initial particle trapping position. For a repulsive external force $\left(\tilde{V}_{p}^{t}>0\right)$ acting against diffusional deposition, the collection efficiency is seen to decay generally exponentially with increasing Peclet number as discussed in the previous section, although no attempt has been made to quantify this observation.

Thus, numerical quadrature of Equations (5.12) and (5.13) using the present Runge-Kutta integration method is seen to converge toward all of the appropriate asymptotic limits as required.

\subsubsection{Efficiency at Intermediate Peclet Numbers}

Although the small- and large-Pe asymptotic limits for the collection efficiency are well described by analytic expressions, the shape of the collection efficiency curves for intermediate Peclet numbers can be quite complex and requires the full numerical integration of Equations (5.12) and (5.13). For example, while the efficiency-curve transition between the small- and large-Pe asymptotic limits is generally monotonic (e.g., Figure 13a for $\tilde{V}_{p}^{t}>0$ or Figure $13 \mathrm{c}$ for $\tilde{V}_{p}^{t}<-0.5$ ), in some cases there may be a local minimum (e.g., Figure 13a for $\tilde{V}_{p}^{t}=-0.1$ ) or maximum (e.g., Figure $13 \mathrm{c}$ for $\left.\tilde{V}_{p}^{t}=-0.1\right)$. The exact shape of the efficiency curve depends on the magnitudes of the three parameters, $\tilde{h}, \tilde{V}_{p}^{t}$, and Pe, and although the interaction among them can be complex, a few simple observations can be made. First, moving the particle trap away from the wafer (i.e., increasing $\tilde{h}$ ) always tends to lower the collection efficiency. Although this effect is most notable for low or intermediate Pe values where diffusional effects are strong, it is also true for large Pe values where deposition is controlled by external forces. The latter claim is supported by noting that gas velocity at the trap location, $\tilde{u}(\tilde{h})$, increases with increasing distance from the wafer so that collection efficiency decreases as shown in the largePe limit given by Equation (5.17). Trap manipulation can be accomplished in practice under some conditions. For example, in plasma processing, the trap location is determined by process parameters such as pressure, rf power, and flow rate; while these parameters may be fixed during etch by process requirements, they could be adjusted to manipulate the particle trap location just prior to plasma ex- 


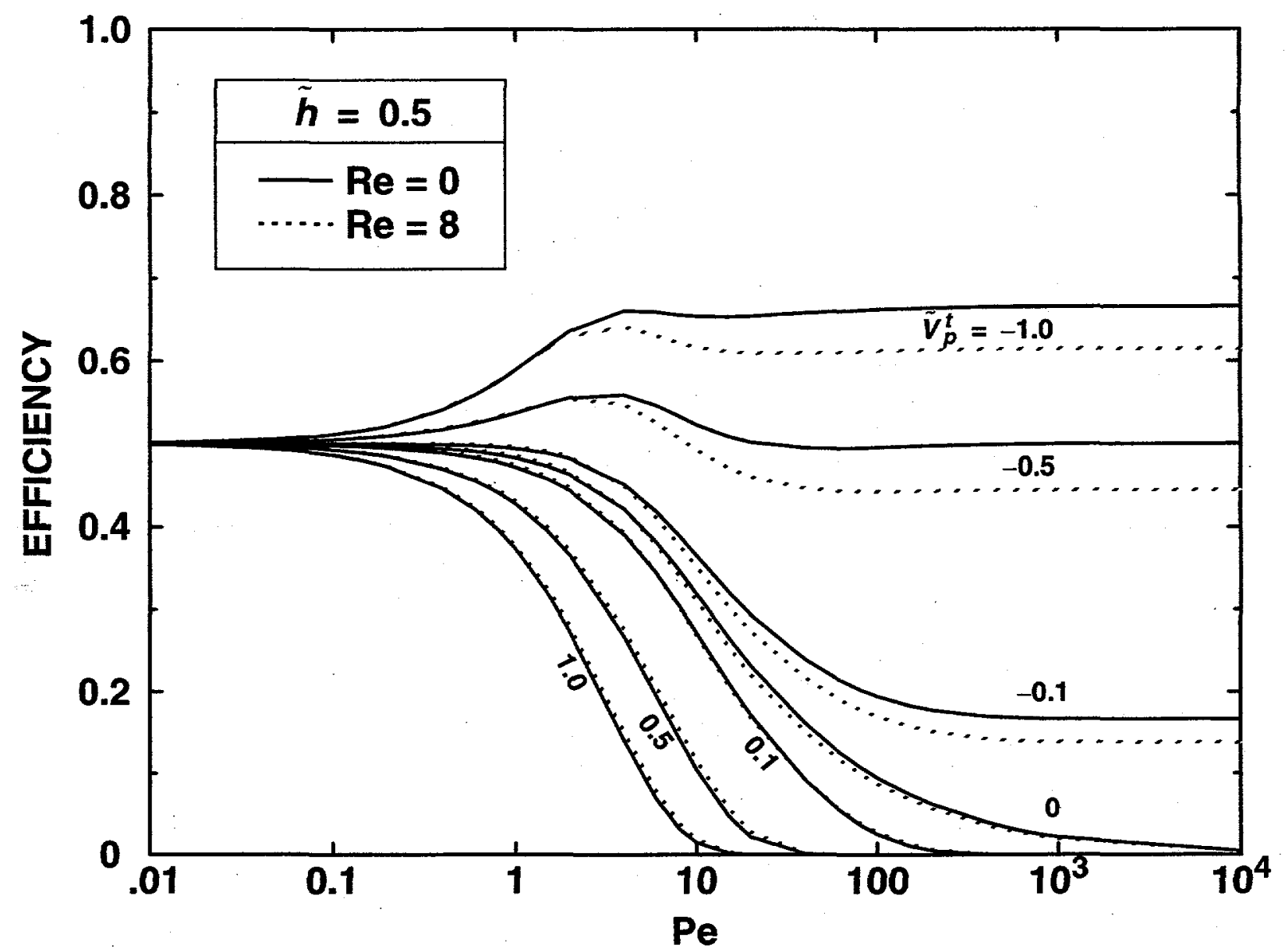

Figure 14. Efficiency vs. Pe for Various Reynolds Numbers. Figures show calculated efficiencies for $\operatorname{Re}=0$ and $2(h / S=0.5)$.

tinction. Similarly, trap position when particle nucleation is present could be controlled by pressure, wall temperature, flow rates, or chemistry selections.

Second, reducing the dimensionless attractive external force or increasing the dimensionless repulsive force always lowers the collection efficiency. This trend is clearly evident in Figure 13 for intermediate and large Peclet numbers, although the benefit becomes less significant at low Pe where diffusion dominates deposition. It is interesting to note that for attractive forces such as gravity (wafer facing up) or thermophoresis (wafer cooler than the surrounding gas), the dimensionless drift velocity increases as pressure is decreased (for constant $U_{o}$ ). In this case, the tendency toward processing at lower pressures ultimately must lead to an increase in the fraction of particles which end up on the wafer. If a process recipe is selected that maintains the wafer warmer than its surroundings, however, the drift velocity resulting from this repulsive external force will increase with decreasing operating pressure (for constant $U_{o}$ ) and thereby reduce the fraction of particles depositing on the wafer.

To explore the effect of Reynolds number on collection efficiency, calculations were made for Reynolds numbers of 0 and 8 using the analytic approximation for the flow field given in Equation (5.1); the results are shown in Figure 14. As seen, even this relatively large variation in Re (spanning the Re

\footnotetext{
4. Note that $\operatorname{Re}=8$ is beyond the range over which the analytic approximation was found to be accurate, but the analytic result is used here beyond its range to qualitatively investigate any Reynolds number dependencies.
} 
range of the majority of low pressure commercial tools) produces only modest variations in the collection efficiency. Reynolds number effects are most apparent in the large-Pe limit for attractive forces. This effect can be quantified by noting that: 1) the large-Pe efficiency limit of Equation (5.17) depends on gas velocity at the trap location, $\tilde{u}(\tilde{h})$, and 2$)$ the value of $\tilde{u}(\tilde{h})$ changes from -0.5 at $\operatorname{Re}=0$ to a value of -0.625 at $R e=8$. This $25 \%$ increase in $\tilde{u}(\tilde{h})$ associated with $\operatorname{Re}$ being increased from 0 to 8 leads to an approximately $25 \%$ decrease in collection efficiency for a weak attractive force, i.e. $\left|\tilde{V}_{p}^{t}\right| \ll|\tilde{u}(\tilde{h})|$. As $\tilde{V}_{p}^{t}$ is increased to a magnitude comparable to $\tilde{u}(\tilde{h})$, this effect diminishes; for example, for $\tilde{V}_{p}^{t}=-1$ the efficiency for $\mathrm{Re}=8$ is only $8 \%$ less than for $\mathrm{Re}=0$.

For small Peclet numbers, the effect of Reynolds number vanishes for all values of the external force. In this case, particle transport by diffusion dominates convective transport, so that the collection efficiency is decoupled from the details of the flow field. Figure 14 also shows that Reynolds effects are negligible in the presence of repulsive external forces; although this conclusion is true in an absolute sense, Reynolds number effects do become important in a relative sense for larger Peclet numbers where the collection efficiency becomes small. For example, for $\tilde{V}_{p}^{t}=1.0$ and a $\mathrm{Pe}=15$, the collection efficiency for $\operatorname{Re}=8\left(\eta=3.18 \cdot 10^{-3}\right)$ is approximately $50 \%$ larger than that for $\operatorname{Re}=0\left(\eta=2.11 \cdot 10^{-3}\right)$. While such differences may not be detectable at low particle concentrations, the difference in the number of particles depositing on the wafer may become quite large when particle concentrations are high - such as is typical of systems in which particle nucleation is occurring.

\subsubsection{Linear Combination of Deposition Terms}

In the earlier work of Cooper et al. (1989) on particle deposition in viscous, axisymmetric stagnationpoint flow of infinite extent over a finite disk, they reported that the net deposition velocity could be approximated as the sum of the deposition velocities for pure diffusion (no external forces) plus pure external force migration (neglecting diffusional effects). For their geometry the diffusional contribution follows from the Levich-Smoluchowski (1962) solution, which in terms of an equivalent deposition velocity is given by $v^{*}=\left(\mathcal{D} / r_{p}\right) \cdot \mathrm{Sh}=\left(\mathcal{D} / r_{p}\right) \cdot 0.616 \cdot \mathrm{Pe}^{1 / 3}$ (note that particle radius, $r_{p}$, was used as the characteristic length in Cooper et al.'s analysis). The external-force driven contribution is the drift velocity $V_{p}^{t}$. In the present geometry, however, approximating the net deposition as a linear combination of these two terms has not proven as successful. One complicating issue is that there are two expressions for the pure diffusion case, Equation (5.15) for the small-Pe limit, and Equation (5.16) for the large-Pe limit, opposed to the single expression from the Levich-Smoluchowski analysis. Physically, the Levich-Smoluchowski allows deposition to increase without bound as the particles become more diffusive (because of the infinite domain), while in the present geometry there is an upper bound to diffusive deposition which is imposed by the finite domain. As mentioned above, for vanishing values of the Peclet number, the appropriate approximation for efficiency is just Equation (5.15).

In the large-Pe limit, with an attractive external force acting, the linear combination of two independent terms does satisfactorily describe the deposition process in some regimes. Here, we approximate the net collection efficiency by $\eta_{\text {net }} \cong\left(\eta_{\text {diffusion }}+\eta_{\text {external }}\right)$, where $\eta_{\text {diffusion }}$ is obtained from Equation (5.16) and $\eta_{\text {external }}$ from Equation (5.17). This linear approximation is found to agree with calculations using numerical quadrature of Equations (5.12) and (5.13) to within $25 \%$ for $\mathrm{Pe}>1000$ in the range $-1<\tilde{V}_{p}^{t}<0$ and $0.3<h<0.99$. For smaller values of the Peclet number or for particle traps closer to the wafer, the error by a simple addition of terms rapidly increases. The linear combination approximation can be extended to smaller values of Pe for weak external forces and for particle traps away from the plates; for example, for $-0.01<\tilde{V}_{p}^{t}<0$ and $0.5<\tilde{h}<0.95$, better than $25 \%$ accuracy is obtained for Peclet numbers larger than about 60.

Although the simple addition of deposition efficiencies by different mechanisms works in some regimes, it is recommended that the collection efficiency be calculated directly from Equations (5.12) and (5.13) for the present parallel-plate geometry. The advent of inexpensive, commercial mathematical analysis packages for desk-top computers makes solution of the required numerical integrals less challenging. 


\subsection{Dimensional Results}

This section presents several example calculations of collection efficiency in dimensional terms when gravity and diffusion act simultaneously. The solution scheme described in the previous section is again used here, except that the dimensional inputs are first converted into the required nondimensional groups before the numerical integrations are performed. The particle diameter replaces the Peclet number as the independent variable in all of the following. All of the examples assume a $200 \mathrm{~mm}$ diameter with a showerhead-to-wafer gap of $2.54 \mathrm{~cm}$. For these calculations, a baseline process is taken as argon flowing at a mass flow rate of $1000 \mathrm{sccm}$ (standard cubic centimeters per minute) at a pressure of 1 torr and temperature of $300 \mathrm{~K}$. Constant gas properties are assumed (isothermal flow) along with a particle density of $1 \mathrm{~g} / \mathrm{cc}$. The Reynolds number for these conditions is 0.984 , which indicates viscous dominated flow and is well inside the range for which the analytic flow field expression can be used. In the following examples, trap height, pressure, flow rate, and pressure are individually varied about the baseline value. Note that in practice these parameters may not be independent; for example, trap height may depend on both pressure and gas flow rate. Finally, the section concludes with a demonstration of the reduction in deposition that can be obtained by using a resistive force, such as heating the wafer relative to the showerhead to take advantage of thermophoretic protection.

The errors associated with neglecting particle inertial effects become significant at the baseline conditions for particle diameters above $\sim 1 \mu \mathrm{m}$. For higher flow rates or at lower pressures, inertial effects become important at even smaller particle sizes. The extension of the present analysis to include inertial effects is not feasible; an alternative is to use Monte Carlo simulations (Brownian dynamics) such as performed for infinite stagnation-point flow by Peters et al. (1989) and for parallel-plate flow by Choi et al. (1996).

\subsubsection{Trap Height Effects}

Plots of calculated particle collection efficiency as a function of particle size are shown in Figure 15 for dimensionless trap heights of $0.1,0.3,0.5,0.7$, and 0.9 . All of the curves exhibit a minimum near $0.1 \mu \mathrm{m}$, with increasing efficiency for both smaller and larger sizes. This shape has commonly been reported in previous deposition studies: for example, see Figure 3 of Cooper et al. (1989) which shows the net stagnation-point deposition velocity based on additivity of convective-diffusion, electrostatic, and gravitational velocities. In Figure 15, the increase in efficiency below $0.1 \mu \mathrm{m}$ is associated with increasing diffusional deposition, while the increase in efficiency above $0.1 \mu \mathrm{m}$ is associated with increasing gravitational deposition. Note that in the parallel-plate geometry the diffusional branch does not increase without bound, but instead asymptotically approaches the highly-diffusive limit $\eta \rightarrow 1-\tilde{h}$ as discussed above. As seen in Figure 15, however, this limit is not quite achieved even for particles as small as $0.001 \mu \mathrm{m}$. As expected based on the previous discussion in nondimensional terms, the trapping height plays a key role in net particle deposition. Clearly, it is always advantageous to manipulate the particle trap to a location as far from the wafer as possible. It is clear from Figure 15 that as the size of IC-killing particles shrinks below $0.1 \mu \mathrm{m}$ that particle collection efficiencies will increase as a result of increased particle diffusion.

\subsubsection{Pressure Effects}

Plots of calculated particle collection efficiency as a function of particle size are shown in Figure 16 for reactor pressures of 1,10100 , and 760 torr. For these calculations the mass flow rate is held constant at the baseline value of $1000 \mathrm{sccm}$, and the trap height is assumed to be half-way between the wafer and showerhead). As before, all of the curves show the characteristic " $U$ " shape resulting from the combination of deposition from convective-diffusion and gravitational settling. For these conditions, it is evident that the diffusional branches of the efficiency curves are nearly independent of pressure. This result is explained by considering that the Peclet number (which along with geometry completely specifies the convective-diffusive problem) depends on the ratio of inlet gas velocity to the particle dif- 


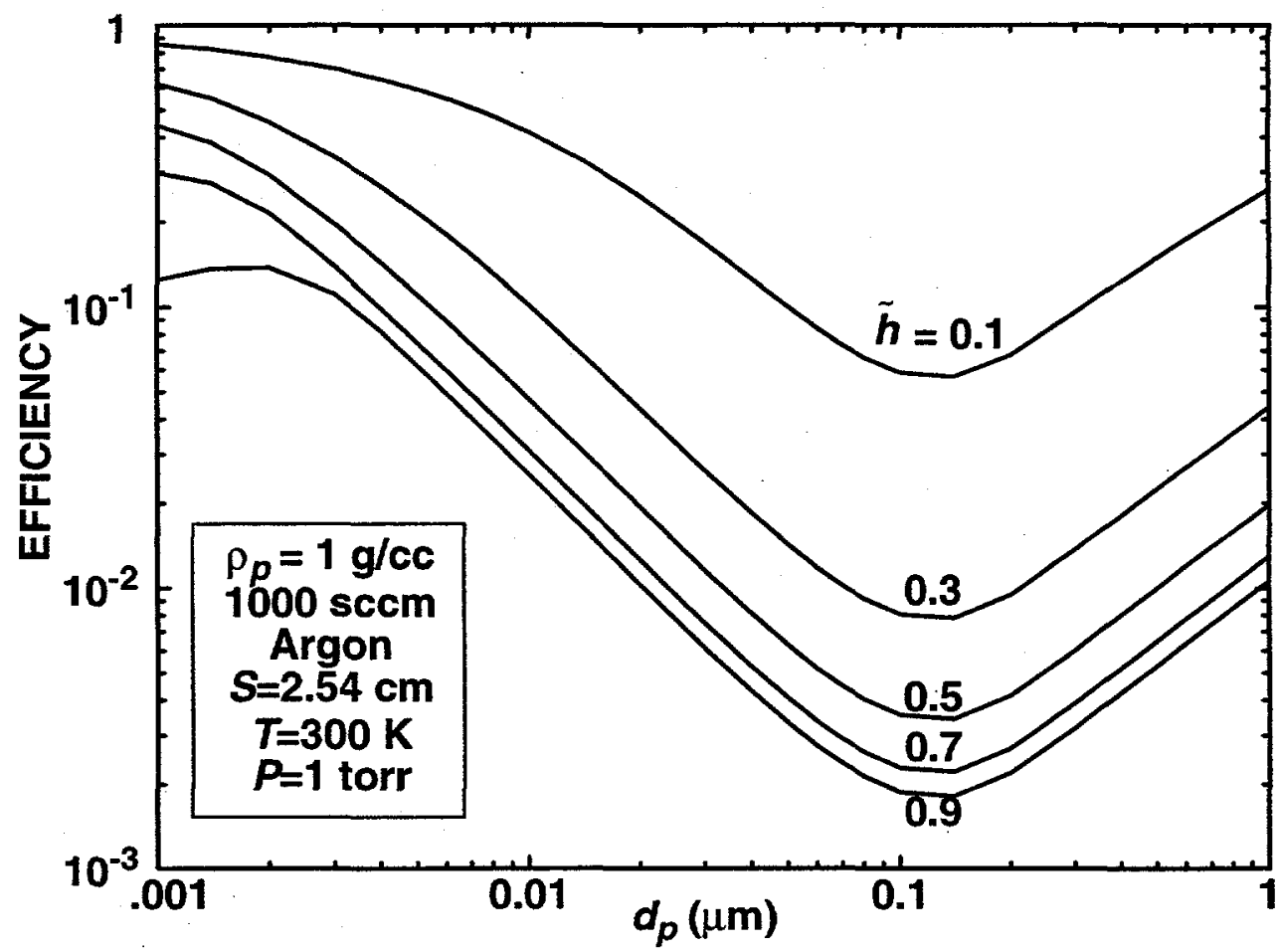

Figure 15. Efficiency vs. particle diameter and trap position (isothermal case). Collection efficiencies for particle transport over a $200 \mathrm{~mm}$ wafer including gravitational settling and diffusion for dimensionless trap locations of $0.1,0.3,0.5,0.7$, and $0.9(S=2.54 \mathrm{~cm}, Q=1000 \mathrm{sccm}$ of argon, $P=1$ torr, $T=300 \mathrm{~K}, \mathrm{Re}=0.98$, and particle density of $1 \mathrm{~g} / \mathrm{cc}$ ).

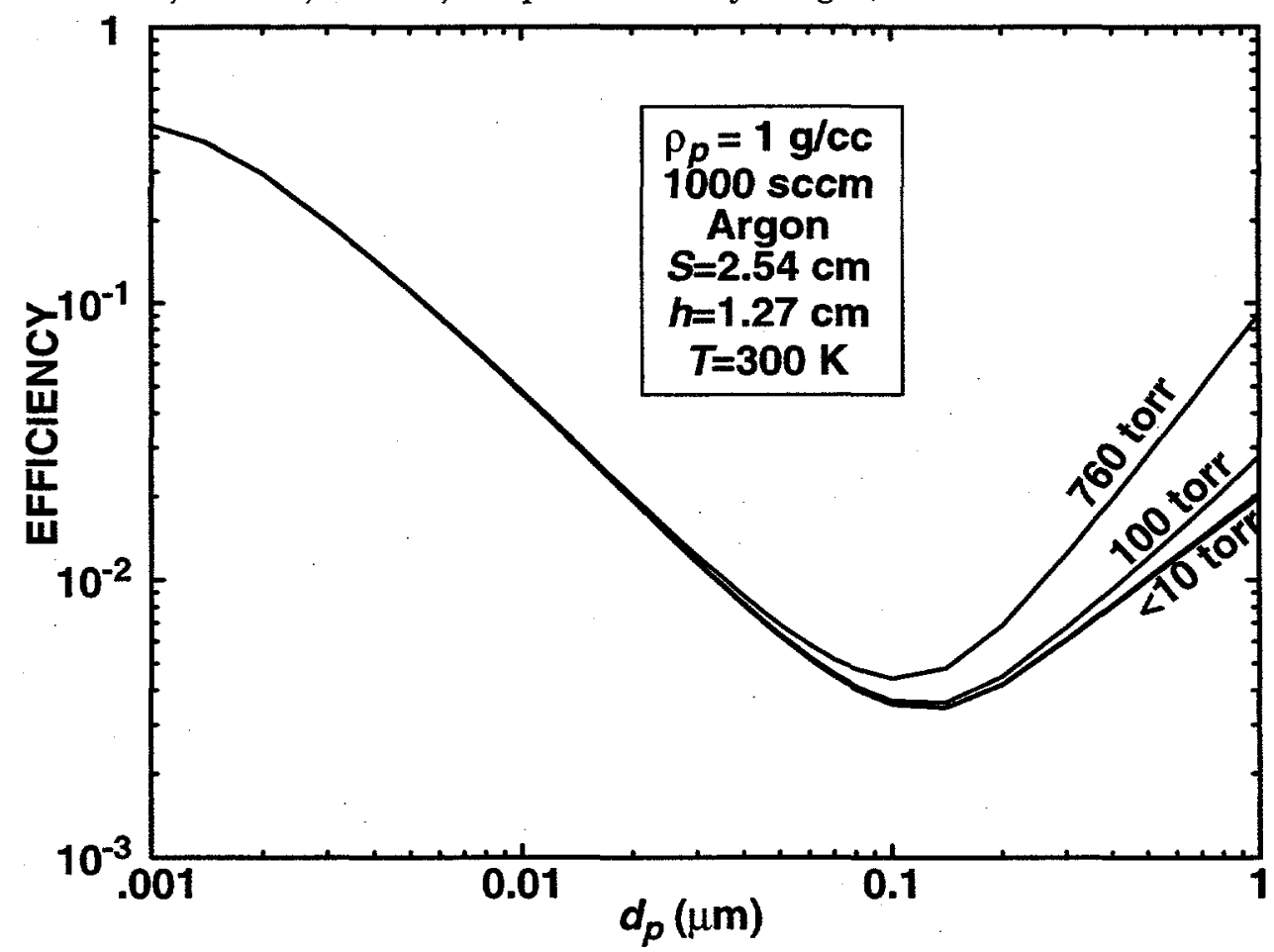

Figure 16. Efficiency vs. particle diameter and pressure (isothermal case). Collection efficiencies for particle transport over a $200 \mathrm{~mm}$ wafer including gravitational settling and diffusion for reactor pressures of $1,10,100$, and 760 torr $(S=2.54 \mathrm{~cm}, Q=1000 \mathrm{sccm}$ of argon, $h=1.27 \mathrm{~cm}, T=300 \mathrm{~K}$, $\operatorname{Re}=0.98$, and particle density of $1 \mathrm{~g} / \mathrm{cc}$ ). 
fusion coefficient, and that both of these quantities are inversely proportional to pressure at a constant mass flow rate. The pressure dependence of the diffusion coefficient is evident in the free molecule limit given in Equation (2.36), which applies at low pressures and for small particle size. The velocity used in scaling the problem, $U_{o}$, is the local gas velocity at the reactor pressure, which also is inversely proportional to pressure at a constant mass flow rate. Thus, as the Peclet number is independent of pressure, the collection efficiency resulting from particle diffusion is nearly independent of pressure for a fixed gas mass flow rate.

The branch of the efficiency curve resulting from gravitational settling is also seen to be independent of pressure below about 10 torr. This result is explained by considering the limiting expression for external-force dominated deposition given by Equation (5.17), which depends only on $\tilde{u}(\tilde{h})$ and $\tilde{V}_{p}^{t}$. The term $\tilde{u}(\tilde{h})$ is independent of pressure as it depends only on trap position and Reynolds number (see Equation (5.1)), and as at constant mass flow rate the Reynolds number is independent of pressure. In the free molecule limit given by Equation (2.28), the gravitational drift velocity varies inversely with pressure so that its ratio to the inlet velocity, $\tilde{V}_{p}^{t}$, must also be independent of pressure. Thus, all of the terms describing the collection efficiency are independent of pressure in the particle free molecule limit. As pressure increases above 10 torr and for particles larger than $1 \mu \mathrm{m}$, however, the free molecule limit for the settling velocity no longer applies and the full expression of Equation (2.26) must be used. As the particle mean free path decreases the inverse pressure dependence of the dimensional settling velocity diminishes, until the continuum regime limit Equation (2.27) is reached which is independent of pressure. As $V_{p}^{t}$ becomes independent of pres-

sure, $\tilde{V}_{p}^{t}$ becomes proportional to pressure, resulting in the marked increase in collection efficiency with pressure as seen in Figure 16 for larger particles and at higher pressures.

\subsubsection{Mass Flow Rate Effects}

Plots of calculated particle collection efficiency as a function of particle size are shown in Figure $\mathbf{1 7}$ for argon mass flow rates of 10,100 , and $1000 \mathrm{sccm}$. For these calculations the reactor pressure is held constant at the baseline value of 1 torr, and the trap height is assumed to be $1.27 \mathrm{~cm}$ (half-way between the wafer and showerhead). Again, all of the curves show the characteristic " $U$ " shape resulting from the combination of deposition from convective-diffusion and gravitational settling. These results show that the collection efficiency is a strong function of gas mass flow rate except for very small particle sizes, for which diffusion dominates and all curves must tend toward the same limit. Note that the point at which the diffusion-dominated limit is achieved varies with mass flow rate: for the lowest flow rate of $10 \mathrm{sccm}$ the limit $(\eta=0.5)$ is reached for particles less than about $0.01 \mu \mathrm{m}$, while at the highest flow rate of $1000 \mathrm{sccm}$ the collection efficiency is still below the limit for $0.001 \mu \mathrm{m}$. Thus, a significant reduction in particle collection efficiency can be achieved by increasing the mass flow rate at constant pressure. The effect of mass flow rate on the actual number of particles depositing on the wafer is less obvious. For example, consider the case in which the mass flow rate is increased from 100 to $1000 \mathrm{sccm}$. Although the collection efficiency drops by approximately a factor of 10 , the flow increases by a factor of 10 , so that if the number of particles entering the domain scales with flow rate than the total number of particles depositing on the wafer should be about the same for the two flow rates. If, however, the number of particles present is independent of the flow rate, then increased flow rates should reduce the number of particles on the wafer.

For calculations where pressure is fixed, it should be noted that the Reynolds number increases proportionately with mass flow. However, even at the highest flow rate considered, 1000 sccm, the Reynolds number is less than one and the analytic creeping flow approximation is excellent.

\subsubsection{Effect of Thermophoresis}

This final section explores the role of thermophoresis in determining particle collection efficiency. For these calculations the showerhead temperature has been held constant at $300 \mathrm{~K}$, and the wafer temperature varied to produce a temperature gradient that drives thermophoretic deposition. To accom- 


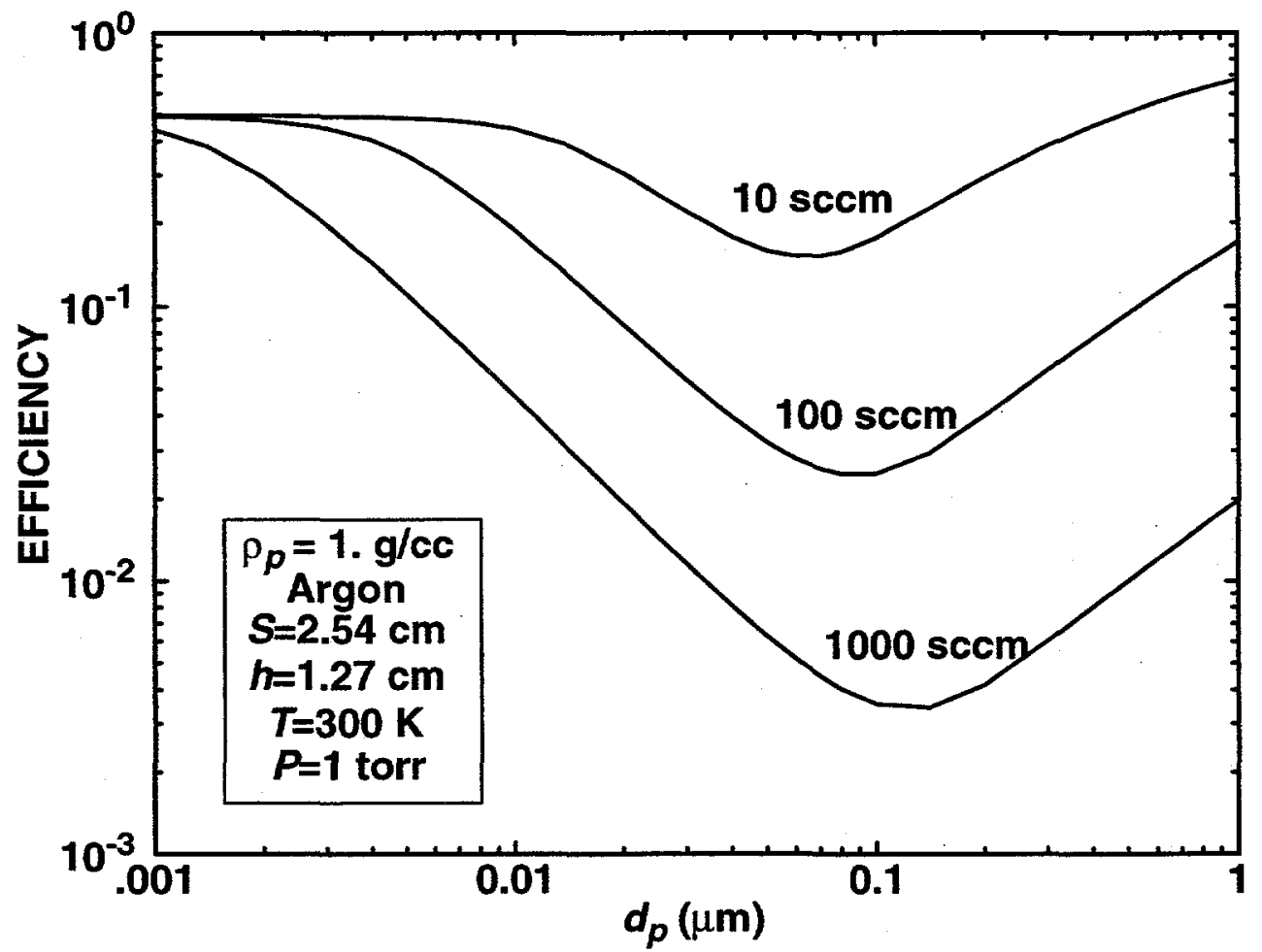

Figure 17. Efficiency vs. particle diameter and flow rate (isothermal case). Collection efficiencies for particle transport over a $200 \mathrm{~mm}$ wafer including gravitational settling and diffusion for gas mass flow rates of 10, 100, and $1000 \mathrm{sccm}$ (argon, $S=2.54 \mathrm{~cm}, h=1.27 \mathrm{~cm}, T=300 \mathrm{~K}$, $P=1$ torr, $0.01<\operatorname{Re}<0.98$, and particle density of $1 \mathrm{~g} / \mathrm{cc}$ ).

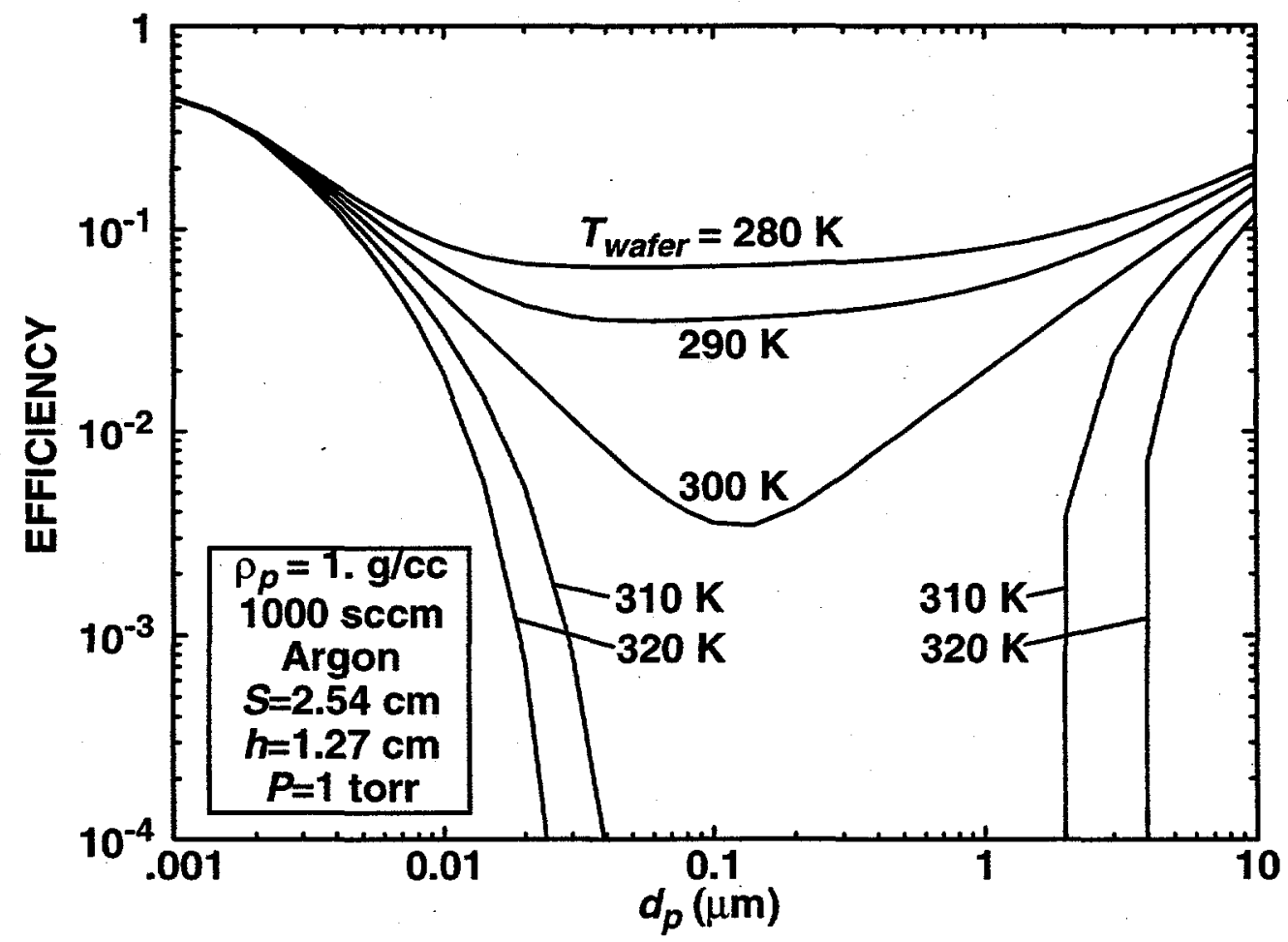

Figure 18. Efficiency vs. particle diameter and wafer temperature. Collection efficiencies including gravity, thermophoresis, and diffusion for wafer temperatures of $280,290,300,310$, and $320 \mathrm{~K}$ (200 mm wafer, $1000 \mathrm{sccm}$ argon, $S=2.54 \mathrm{~cm}, h=1.27 \mathrm{~cm}, T_{\text {showerhead }}=300 \mathrm{~K}, P=1 \mathrm{torr}$, and particle density of $1 \mathrm{~g} / \mathrm{cc}$ ). 
modate our assumption of constant properties, only small temperature differences are considered. Plots of calculated particle collection efficiency as a function of particle size are shown in Figure 18 for wafer temperatures of $280,290,300,310$, and $320 \mathrm{~K}$. The baseline conditions described above are used for all of these calculations: reactor pressure 1 torr, argon mass flow rate of $1000 \mathrm{sccm}$, wafer-toshowerhead gap of $2.54 \mathrm{~cm}$, and the trap height is assumed to be $1.27 \mathrm{~cm}$. A particle of density $1 \mathrm{~g} / \mathrm{cc}$ was assumed, and the ratio of the gas to particle thermal conductivity was taken as 0.02 to approximate a fused silica particle. As before, all of the curves show the characteristic " $U$ " shape resulting from the combination of deposition from convective-diffusion and external forces, where the net external forces contains contributions from both gravitational and thermophoretic forces. The minimum collection efficiency for the case of thermophoretic protection (wafer hotter than showerhead) becomes vanishingly small and so is not shown; note that even for thermophoretic protection the collection efficiency never exactly reaches zero as there always is some contribution from diffusion.

A key result of these calculations is that even modest temperature differences can lead to dramatic changes in collection efficiency. For example, deposition is nearly eliminated in the $0.1-1.0 \mu \mathrm{m}$ range when the wafer is kept only 10 to 20 degrees warmer than the showerhead. On the other hand, the collection efficiency increases by an order of magnitude when the wafer temperature is decreased 10 degrees as compared to the isothermal case. In addition, when the wafer temperature is kept below the showerhead temperature, the depth of the collection efficiency minimum at about $0.1 \mu \mathrm{m}$ becomes shallower; this results from the fact that the thermophoretic drift velocity is nearly independent of particle diameter as indicated by Equations (2.30) and (2.31). These calculations clearly demonstrate the importance of keeping the wafer warmer than its surroundings at all times.

\subsection{Summary}

This chapter explored particle deposition resulting from the simultaneous effect of external forces and Brownian motion in a parallel-plate geometry characteristic of a wide range of semiconductor process tools. The need to properly account for diffusion-enhanced particle deposition becomes increasingly important as the semiconductor industry moves toward smaller feature sizes and becomes concerned with smaller-sized particles. Particle transport was modeled using the Eulerian approach of Section 2.6 , so that the continuum convective-diffusion equation was solved for particle flux. One strength of the Eulerian formulation is in predicting particle transport resulting from the combination of applied external forces (including the fluid drag force) and the chaotic effect of particle Brownian motion (i.e., particle diffusion), although the current implementation neglected particle inertia. Furthermore, particles were assumed to originate in a planar trap located between the plates, and only transport in the inter-plate region was considered (showerhead acceleration is neglected). Flow between infinite parallel plates was assumed as described by the quasi-1-D creeping flow approximation of Chapter 3, where the showerhead was treated as a porous plate.

The key result of the present analysis was the particle collection efficiency - which is the fraction of trapped particles which end up on the wafer. An analytic, integral expression was derived that gives the particle collection efficiency as a function of four dimensionless parameters: $\operatorname{Re}, \tilde{h}, \tilde{V}_{p}^{t}$, and $\operatorname{Pe}(\mathrm{a}$ fifth - the interception parameter $d_{p} / S$ - was neglected in this chapter). The first parameter, the Reynolds number, completely specifies the flow field under the present assumptions. The second parameter, the dimensionless trap height, $\tilde{h}=h / S$, specifies the position of the particle source term. The influence of external forces enters through the third parameter, the dimensionless particle drift velocity, $\tilde{V}_{p}^{t}=V_{p}^{t} / U_{o}$, which is defined as the $z$-component of the net drift velocity. The fourth parameter is the particle Peclet number, $\mathrm{Pe}=S U_{o} / \mathcal{D}$, which is a measure of the relative importance of particle Brownian motion. In the free molecular limit the Peclet number is proportional to diameter squared, so that $\mathrm{Pe}^{1 / 2}$ can be thought of as a dimensionless particle size.

The theoretical, asymptotic limits of the analytic, integral expression given by Equations (5.12) and (5.13) were considered. The important results were that the collection efficiency tends to: 1) zero or one as the trap approaches the showerhead or wafer, respectively; 2 ) $1-\tilde{h}$ in the highly diffusive, 
small-Peclet number limit, and 3) $\left|\tilde{V}_{p}^{t}\right| /\left(\left|\tilde{V}_{p}^{t}\right|+|\tilde{u}(\tilde{h})|\right)$ in the large-Pe limit where an attractive external force dominates diffusion. Practically, the first two limits support imply that particle transport to the wafer is always reduced by pushing the trap as far as possible from the wafer. The third limit has already been derived in Chapter 4 for the case where particle diffusion can be neglected.

Numerical quadrature of Equations (5.12) and (5.13) using a fourth-order Runge-Kutta technique was used to calculate particle collection efficiency in terms of the controlling dimensionless parameters. Initial calculations showed the numerical results to be in good agreement with the various analytic limits, providing confidence in the current implementation. In general, the highly-diffusive limit was approached for Peclet numbers less than about 0.1, while the nondiffusing limit was essentially reached for Peclet numbers larger than $\sim 10^{2}$ or $\sim 10^{3}$ depending on the strength of the external force and the initial particle trapping position. For intermediate Peclet numbers, the shapes of the collection efficiency curves were often found to be complex; for example, some conditions gave efficiency curves which showed local minima or maxima. Despite this complexity, a few simple observations were made: moving the particle trap away from the wafer (i.e., increasing $\tilde{h}$ ) always lowered the collection efficiency, as did reducing the attractive external force or increasing the repulsive force. Finally, calculations made for Reynolds numbers of 0 and 8 showed only modest variations in particle collection efficiency, suggesting that this parameter plays only a minor role over the range likely to be encountered in realistic processing environments.

Based on the present results, the strategy of approximating net deposition by the linear combination of purely diffusive and purely attractive external-force deposition terms has not proven as successful as for unbounded, viscous axisymmetric stagnation-point flow (Cooper et al., 1989). One complicating issue in the present case is that there are two distinct limits (for the small- and large-Pe cases), opposed to the single expression from the Levich-Smoluchowski analysis. Physically, the Levich-Smoluchowski allows deposition to increase without bound as the particles become more diffusive (because of the infinite domain), while in the present geometry there is an upper bound to diffusive deposition which is imposed by the finite domain. Thus, although the simple addition of deposition efficiencies by different mechanisms can prove satisfactory in some regimes, it is recommended that the collection efficiency be calculated directly from Equations (5.12) and (5.13) for the present parallel-plate geometry. The advent of commercial mathematical analysis packages makes solution of equations containing numerical integrals less challenging.

Example calculations of collection efficiency were also presented in dimensional terms for one representative set of process conditions $(200 \mathrm{~mm}$ wafer, showerhead-to-wafer gap of $2.54 \mathrm{~cm}$, mass flow rate of $1000 \mathrm{sccm}$ argon, 1 torr, $300 \mathrm{~K}$ ). In all cases, the efficiency curves exhibited a minimum near $0.1 \mu \mathrm{m}$, with increasing efficiency for both smaller and larger sizes. Trapping height was found to play a key role in deposition, and in all cases it was advantageous to manipulate the particle trap to a location as far from the wafer as possible. Trap manipulation can be accomplished in practice under some conditions. For example, in plasma processing, the trap location is determined by process parameters such as pressure, rf power, and flow rate; while these parameters may be fixed during etch by process requirements, they could be adjusted to manipulate the particle trap location just prior to plasma extinction. Similarly, trap position when particle nucleation is present could be controlled by pressure, wall temperature, flow rates, or chemistry selections.

At constant mass flow rate, collection efficiency was found to be independent of pressure for the low pressures and small particle sizes of interest. At constant pressure, collection efficiency was found to decrease significantly with increasing mass flow rate. Thus, from a particle transport point of view, reduction of particle-on-wafer counts could be obtained by increasing the mass flow rate in a process. Another key result was that even modest temperature differences can lead to dramatic changes in collection efficiency due to the thermophoretic force. For example, deposition is nearly eliminated when the wafer is kept only 10 to 20 degrees warmer than the showerhead. On the other hand, the collection efficiency increases by an order of magnitude when the wafer temperature is decreased 10 degrees below the showerhead temperature. Caution is suggested in implementing any of these strategy as the effect on the particle source term is not known. 


\section{Inertia-Enhanced Deposition}

As discussed in the introduction, the use of a showerhead restricts the area available to the flow inside the showerhead; consequently, the velocity of the gas inside the holes is much larger than the face velocity in the gap below. Particles originating upstream of the showerhead and suspended in the flow can be dramatically accelerated while passing through the showerhead, so that at the exit of the showerhead particle velocities much larger than the fluid face velocity are possible. Depending on conditions, particle acceleration by the showerhead can lead to inertia-enhanced particle deposition on the wafer below (Rader and Geller, 1998). Thus, a complete description of particle deposition on a wafer in a parallel-plate reactor must include a description of particle transport through the showerhead as well as an analysis of particle transport in the inter-plate region.

This chapter explores the role of inertia-enhanced deposition using the Lagrangian particle transport formulation given in Section 2.2. The strength of the Lagrangian formulation is in predicting particle transport resulting from the combination of applied external forces and particle inertia; the current implementation does not account for particle diffusion. The problem is separated into two domains in which particle and fluid transport are determined: 1) within a showerhead-hole, and 2) between the showerhead and wafer. The fluid transport in both domains has already been treated in Chapter 3 . For flow in the showerhead, analytic expressions for the velocity profile were derived for plug flow (Equation (3.3)) and fully developed parabolic flow (Equation (3.4)). In Section 6.1, these velocity profiles will be used to calculate particle acceleration while passing through the showerhead. The result of this analysis will be an analytic expression for the dimensionless particle velocity at the exit of the showerhead, as a function of showerhead geometry, dimensionless jet velocity, and particle Stokes number. To improve generality, the results of Section 6.1 are presented in nondimensional form.

A Lagrangian analysis of particle transport in the inter-plate region is presented next in Section 6.2. The desired result is a determination of whether or not a particle entering the domain through the showerhead ultimately deposits on the wafer; this determination requires an integration of the particle equations of motion - Equations (2.2) and (2.3). As the particle trajectory is strongly influenced by the flow through the drag force, a description of the inter-plate flow field is also needed; both the numerical and analytic flowfields discussed in Section 3.4 are used. For this analysis, the particle initial velocity (at the showerhead exit) is treated as a parameter, i.e., it is not calculated by the method of Section 6.1. The result of this analysis is a determination of the particle collection efficiency as a function of dimensionless particle initial velocity, reactor geometry, inter-plate Reynolds number, particle Stokes number, and dimensionless particle drift velocity. As before, all of the discussion is presented in nondimensional form.

Section 6.3 explores coupled transport where the effects of particle acceleration in the showerhead are combined with inter-plate transport. The result of this analysis is a determination of the particle collection efficiency as a function of showerhead and reactor geometry, the dimensionless jet velocity, inter-plate Reynolds number, particle Stokes number, and dimensionless particle drift velocity. The methods of Section 6.1 are first used to calculate the particle velocity at the showerhead exit; this result is then used as the particle initial velocity for the trajectory calculations presented in Section 6.2. All of the results in Section 6.3 are presented in nondimensional form.

To explore the implications of inertia-enhanced deposition in dimensional terms, Section 6.4 presents several illustrative examples of particle deposition using realistic reactor dimensions and process parameters. To simplify discussion, free molecule limits are referred to throughout this chapter for all particle force and drift velocity expressions; this approximation is warranted by the low operating pressures and small particle sizes of interest in semiconductor manufacturing defect control. In the dimensional plots, however, the complete expressions for forces and drift velocities have been used for the calculations. The chapter concludes with a list of design guidelines for reducing particle deposition in semiconductor manufacturing tools. 


\subsection{Particle Transport in the Showerhead Holes}

For this analysis, particles are assumed to be evenly distributed across the showerhead hole inlet and to enter with zero radial velocity and with an initial axial velocity equal to the face velocity $U_{o}$. The particle will immediately see a fluid velocity $U_{j e t}(r)$, and will either be accelerated or deaccelerated by fluid drag depending on the magnitude of $U_{j e t}$. Because of inertia, however, the particle will require a finite time to respond: in particular, the particle will accelerate to $U_{j e t}$ only if the showerhead hole is sufficiently long or the particle sufficiently small. In practice, the particle velocity at the exit of the showerhead, $V_{p o}$, will fall somewhere between $U_{o}$ and $U_{j e t}$; depending on the relative magnitudes of these two velocities, the showerhead thickness, and the particle response time $\tau$.

Assuming fully developed flow at the inlet and neglecting lift forces, the particle will remain at its initial radial position while in the tube; consequently, the axial fluid velocity driving the particle through the tube will also remain constant during the traverse. For plug flow, all particles will be accelerated by the same fluid velocity, $\bar{U}_{j e t}$, independent of radial starting position. For fully-developed parabolic flow, the local fluid velocity for each particle will depend on its starting position; particles near the wall will be slowed by drag, while particles near the centerline will be significantly accelerated. For parabolic flow, the assumption that the particles are evenly distributed (i.e., that the particle flux is constant) across the tube inlet is based on the assumption that the fluid entrance length is so short that particle do not have time to migrate radially as the flow is developing. ${ }^{1}$

The problem of a particle of given initial velocity experiencing a step-function change in the local fluid velocity is a classic problem. Although the problem could be solved in dimensional form, it is convenient for matching with the second part of this analysis if we solve the nondimensionalized particle equations of motion, Equations (2.19) and (2.20). As stated previously, we choose the mean chamber axial velocity $U_{o}$ as the characteristic velocity and the inter-plate spacing $S$ as the characteristic length scale. External forces are assumed negligible compared to the large drag forces encountered in the showerhead holes. Given a particle of Stokes number St entering the hole with initial velocity $U_{o}$, and an axially-constant fluid velocity $U_{\text {jet }}(r)$, Equations (2.19) and (2.20) can be solved analytically for the particle velocity at the showerhead exit, $z=L$ :

$$
\begin{gathered}
\frac{L}{S}=\frac{U_{j e t}}{U_{o}} \tilde{t}+\operatorname{St}\left(\frac{U_{j e t}}{U_{o}}-1\right)\left(e^{-\frac{\tilde{t}}{\mathrm{St}}}-1\right) \\
\frac{V_{p o}}{U_{o}}=\frac{U_{j e t}}{U_{o}}-\left(\frac{U_{j e t}}{U_{o}}-1\right) e^{-\frac{\tilde{t}}{\mathrm{St}}}
\end{gathered}
$$

The solution procedure is as follows: 1 ) calculate $U_{j e t} / U_{o}, L / S$, and St from process and particle parameters, 2) solve Equation (6.1) for the dimensionless time $\tilde{t}$, and 3) use $\tilde{t}$ in Equation (6.2) to solve for the dimensionless particle velocity at the showerhead exit. Equation (6.1) must be solved iteratively; for this purpose we use a Newton root-finding technique.

Some complexity has been added to Equations (6.1) and (6.2) by our use of one characteristic length and one characteristic velocity for both the showerhead and parallel-plate domains. An interesting result is found if $L$ and $U_{j e t}$ (natural choices for characterizing transport through the showerhead) are substituted for $S$ and $U_{o}$ as the characteristic length and velocity. With the appropriate redefinitions of the nondimensional terms, a set of equations similar to Equations (2.19) and (2.20) can be derived that depend on the jet Stokes number, which is related to our earlier definition by:

\footnotetext{
1. An alternate assumption is that the particles follow streamlines, in which case the particle concentration is constant across the tube inlet so that the local flux of particles through the tube depends on radial position.
} 


$$
\mathrm{St}_{j e t}=\frac{\tau U_{j e t}}{L}=\mathrm{St} \frac{U_{j e t}}{U_{o}} \frac{S}{L} .
$$

Using the same assumptions and initial conditions as above, we can derive a set of equations analogous to Equations (6.1) and (6.2) that are independent of $L / S$, i.e. $V_{p o} / U_{j e t}=f\left(U_{j e t} / U_{o} \mathrm{St}_{j e t}\right)$. In addition, the functional dependence on the velocity ratio $U_{j e t} / U_{o}$ is very weak (entering only as a result of the assumption that the initial particle velocity is $U_{o}$ ), and vanishes for the limiting case where $U_{j e t} / U_{o} \gg 1$ (which includes the case where the particle initial velocity is zero). In this limiting case, the ratio of the showerhead-exit velocity of the particle to $U_{\text {jet }}$ depends only on the jet Stokes number. This result suggests plotting the results of Equations (6.1) and (6.2) as $V_{p o} / U_{j e t}$ against $S t_{j e t}$ - such as shown in Figure 19. As can be seen, the dimensionless particle velocity at the exit of the showerhead, $V_{p o} / U_{j e t}$, is reasonably insensitive to the velocity ratio when $U_{j e t} / U_{o}>10$.

Thus, the analysis of particle transport in the showerhead domain is complete: given the three inputs $\bar{U}_{j e t} / U_{o} L / S$, and St (and particle initial radial position to determine $U_{j e t}(r)$ for parabolic flow), we can calculate the dimensionless velocity $V_{p o} / U_{o}$ at which the particle exits the showerhead. Interestingly, both the mean velocity ratio and the length ratio depend only on reactor geometry; process conditions such as temperature, pressure, or flow rate enter only through the Stokes number. Thus, for a given Stokes number, the extent of particle acceleration in the showerhead is entirely determined by hardware and is thus a characteristic of a specific tool design. Finally, in moving to the calculation of particle trajectories between the plates, the sign of the particle velocity $V_{p o} / U_{j e t}$ must be switched to account for the different coordinate systems used in the two domains (see Figure $\mathbf{1 b}$ and inset).

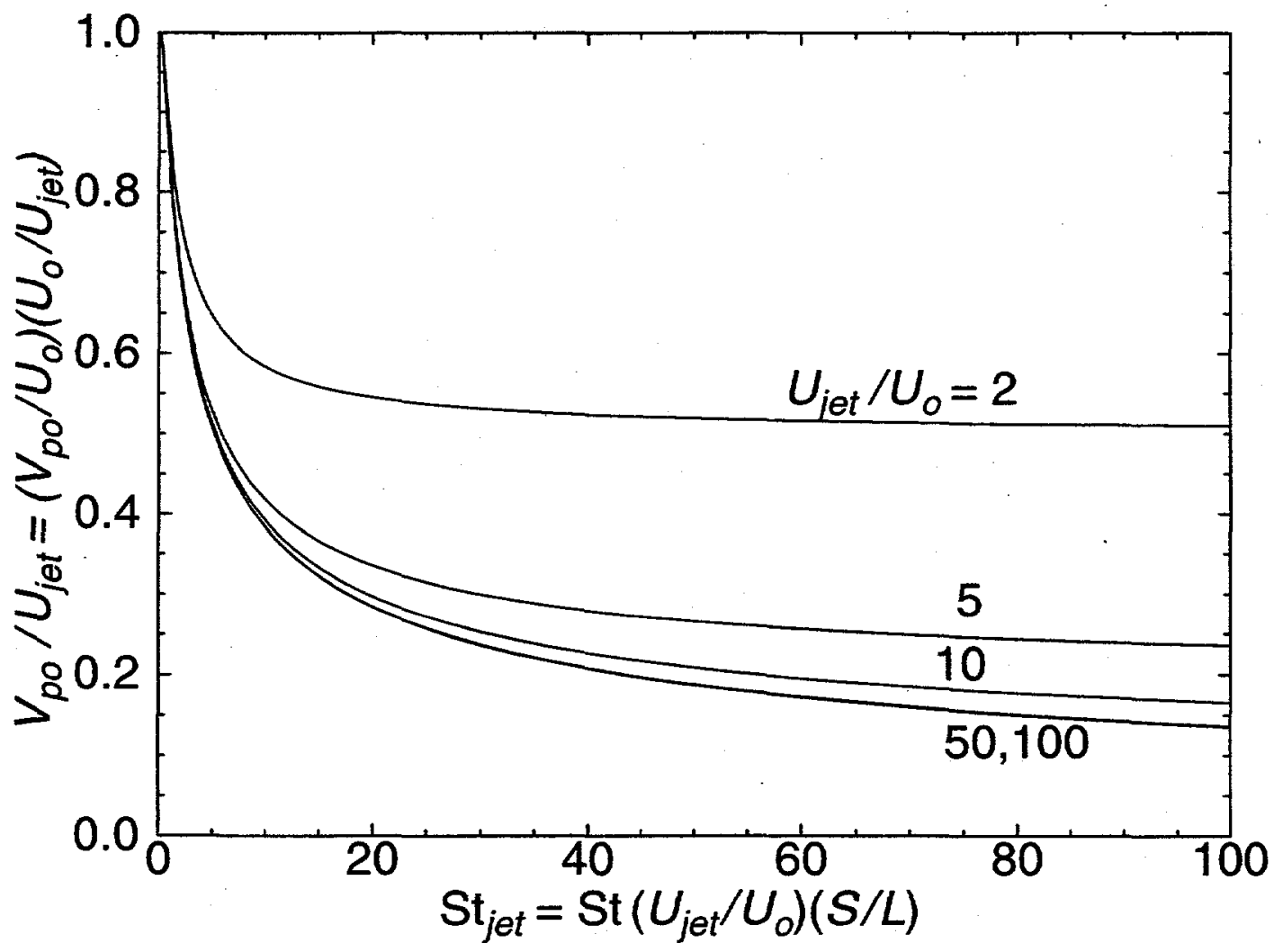

Figure 19. Acceleration of Particles Through Showerhead. Dimensionless velocity of particles exiting showerhead tubes, $V_{p d} / U_{j e t}$, as a function of jet Stokes number, $\mathrm{St}_{j e t}$, for a range of velocity ratios $\left(U_{j e t} / U_{o}=2,5,10,50\right.$, and 100$)$. 


\subsection{Particle Transport between Parallel Plates}

In this section, a Lagrangian formulation is used to calculate particle trajectories in the inter-plate region using both numerical FIDAP and analytic solutions of the flow field. For numerical simulations of flow between finite parallel plates, we used the built-in particle tracking capabilities of FIDAP 7.0. The FIDAP implementation allows the user to start particles with user-defined initial positions and velocities; FIDAP then integrates the particle equations of motion - Equations (2.2) and (2.3) - to calculate the particle trajectory through the inter-plate region. The particles are typically started at the showerhead exit; the initial velocity is treated in this section as a parameter (later the initial particle velocity will be determined by the methods of Section 6.1 ). For all of the trajectory calculations, a large aspect ratio $\left(R_{W} / S=12\right)$ geometry was used so that the flow was 1-D throughout most of the domain.

FIDAP requires that the user specify a variety of parameters which control the particle integration method. In the present work, a range of these parameters were tested to assure that accurate particle paths were obtained. For the flow parameters and geometry used in this study, an explicit integration algorithm with a variable time step and global error control was found to provide satisfactory accuracy with the following parameters: VARIABLE $=2 . \times 10^{-5}$, WINDOW $=0.4$, and UCHAR=VCHAR $=0.001 .^{2}$ An initial time step of $1 . \times 10^{-4}$ was selected - this time step was automatically adjusted by FIDAP during the integration to provide the required level of error control. A generalized drag coefficient with $\mathrm{ACOEF}=1$ was selected, which results in Stokes drag law being used. Because of inherent limitations in the FIDAP integration algorithm, reported efficiency values are typically accurate to three significant figures.

For the asymptotic flow result given by Equation (3.5), particle collection efficiencies were calculated using a stand-alone code that uses a fourth order Runge-Kutta scheme to obtain particle trajectories by integration of Equations (2.19) and (2.20). The integrator includes automatic error control features, and residual and cumulative error tolerances of $10^{-10}$ provide accurate particle tracking; reducing error tolerances below this level did not change calculated efficiencies to five significant figures. The algorithm allowed for particle interception effects, in that the particle was considered collected if it approached within one particle radius of the surface. For the calculations presented in this section, though, an artificially small particle diameter was used $\left(d_{p} / S=1 . \times 10^{-7}\right)$ so that the interception effect was negligible.

\subsubsection{General Results}

In this section we present an overview of particle transport between the plates. Under the present assumptions, four dimensionless parameters uniquely determine particle transport in the inter-plate region: Re, St, $V_{p}{ }^{t} / U_{o}$, and $V_{p o} / U_{o}$ (a fifth - the interception parameter $d_{p} / S$ - is neglected in this section). The first parameter, the Reynolds number, completely specifies the flow field for the infinite parallel-plate geometry - as demonstrated in the analytic low-Re approximation to the flow field given in Equation (3.5). For a finite-plate geometry, the aspect ratio is also needed to specify the flow. The second parameter, the particle Stokes number, is used in this work as a dimensionless particle diameter - as suggested by the free molecule limit Equation (2.23). The influence of external forces enters through the third parameter, the dimensionless particle drift velocity, which parameterizes the forces via the $z$-component of the net drift velocity, $V_{p}{ }^{t}$. The fourth parameter, the dimensionless particle velocity at the showerhead exit, is determined by the strength of the showerhead acceleration effect as. described in Section 6.1. In this section, however, $V_{p o} / U_{o}$ will be taken as an independent variable; in the following section we discuss the coupling of the showerhead and parallel-plates domains.

\footnotetext{
2. The error control approach in FIDAP is not straightforward and the link between the reported parameters and some absolute measure of accuracy is not obvious. In all cases particle trajectories were repeated with increasingly stringent error tolerances until converged results were obtained. The values of the parameters which provided sufficient accuracy are specific to this problem, and are reported here for archival purposes.
} 
The effect of the these dimensionless parameters is shown in Figure 20, where particle collection efficiency is plotted against Stokes number for $\mathrm{Re}=8$. For Figure $20 \mathrm{a}$, an initial dimensionless particle velocity $V_{p o} / U_{o}=-1$ is assumed (no particle showerhead acceleration), while in Figure $20 \mathrm{~b}$ the initial velocity is taken as -100 (substantial showerhead acceleration characteristic of commercial reactors). In each plot the influence of external forces is explored by varying the drift velocity: curves for $V_{p}^{t} / U_{o}$ $=-0.5,-0.1,-0.01,0,0.1$, and 1.0 are shown. Negative values of the drift velocity correspond to an external force directed towards the wafer (attractive, enhancing deposition), while positive values correspond to an external force directed away from the wafer (repulsive, inhibiting deposition). Several important features of these plots will now be explored.

First, inertial effects lead to particle deposition even in the absence of external forces as shown by the curves for $V_{p}{ }^{t} / U_{o}=0$ in Figure 20. In this case there is a critical Stokes number, $\mathrm{St}_{\text {crit }}$, below which no deposition occurs. At $\mathrm{St}_{\text {crit }}$ there is a sharp jump in efficiency, which then increases towards unity with increasing St. The jump is steeper, higher (approaching unit collection efficiency) and occurs for a much smaller $\mathrm{St}_{\text {crit }}$ in the case with substantial showerhead acceleration than when the particles enter with the fluid face velocity. This effect is discussed in greater detail below. As seen in Figure 20, particle inertia can also lead to deposition even when an external force is pushing particles away $\left(V_{p}^{t} / U_{o}>0\right)$. The extent of external force "protection" is significantly reduced for large initial velocities: compare the $V_{p o} / U_{o}=0$ and 1 curves in Figure 20a and b.

Second, when an external force is directed toward the wafer $\left(V_{p}^{t} / U_{o}<0\right)$, particle deposition occurs at all values of the Stokes number. In the small-St limit (negligible particle inertia), the collection efficiency should tend to Equation (4.2) for external forces that can be described by a potential; this trend is clearly evident in Figure 20. For example, for $V_{p}{ }^{t} / U_{o}=-0.5$, Equation (4.2) predicts an efficiency of $1 / 3$ which is the observed asymptote of the appropriate curves in Figure 20a and $b$. The presence of an attractive external force smooths the shape of the efficiency curves for large attractive forces; however, when the magnitude of the attractive force is small (e.g., $\left.V_{p}{ }^{t} / U_{o}=-0.01\right)$, the efficiency still exhibits a sharp increase in the neighborhood of $\mathrm{St}_{\text {crit }}$ (from the no-force case). The rise in efficiency near $\mathrm{St}_{\text {crit }}$ is much steeper for high initial particle velocities.

Finally, in the large-St limit, the collection efficiency must approach unity for all cases. That is, for large enough Stokes numbers, particle inertia leads to straight trajectories and complete deposition independent of the details of the flow field, the external forces, or particle initial velocity (assuming it is not zero). This limit is approached in all of the calculations shown in Figure 20.

In the remainder of this section, we will explore in greater detail three specific aspects of particle transport between parallel plates: 1) comparison of the numerical and analytic solutions for the flow field, 2) effect of fluid Reynolds number, and 3) asymptotic limit of $\mathrm{St}_{c r i t}$ in the limit of large particle initial velocity.

\subsubsection{Comparison of Efficiencies using Numerical and Analytic Flow Solutions}

To determine the accuracy of particle transport calculations using the low-Re analytic flowfield approximation, collection efficiency results using Equation (3.5) with the Runge-Kutta particle trajectory solver were compared to those calculated with FIDAP. For the FIDAP tests, the particle trajectories were calculated in the central region of the domain where edge effects were insignificant. Since the greatest discrepancy between the analytic and numerical velocity fields occurs at higher Reynolds numbers, efficiency calculations were made with the two techniques for a Reynolds number of eight (the highest value used in this study). The results are shown in Figure 20. Calculations with the analytic approximation are shown as solid lines, while the FIDAP calculations are shown as open symbols. Good agreement is found between the two techniques with efficiencies for the same Stokes number differing by at most $2 \%$. This result is not too surprising, as the analytic flow field typically agreed with the FIDAP result within 5\% everywhere except for the radial velocity near the inlet, where the radial velocity is near zero and consequently has little effect on particle trajectories. In par- 
a)

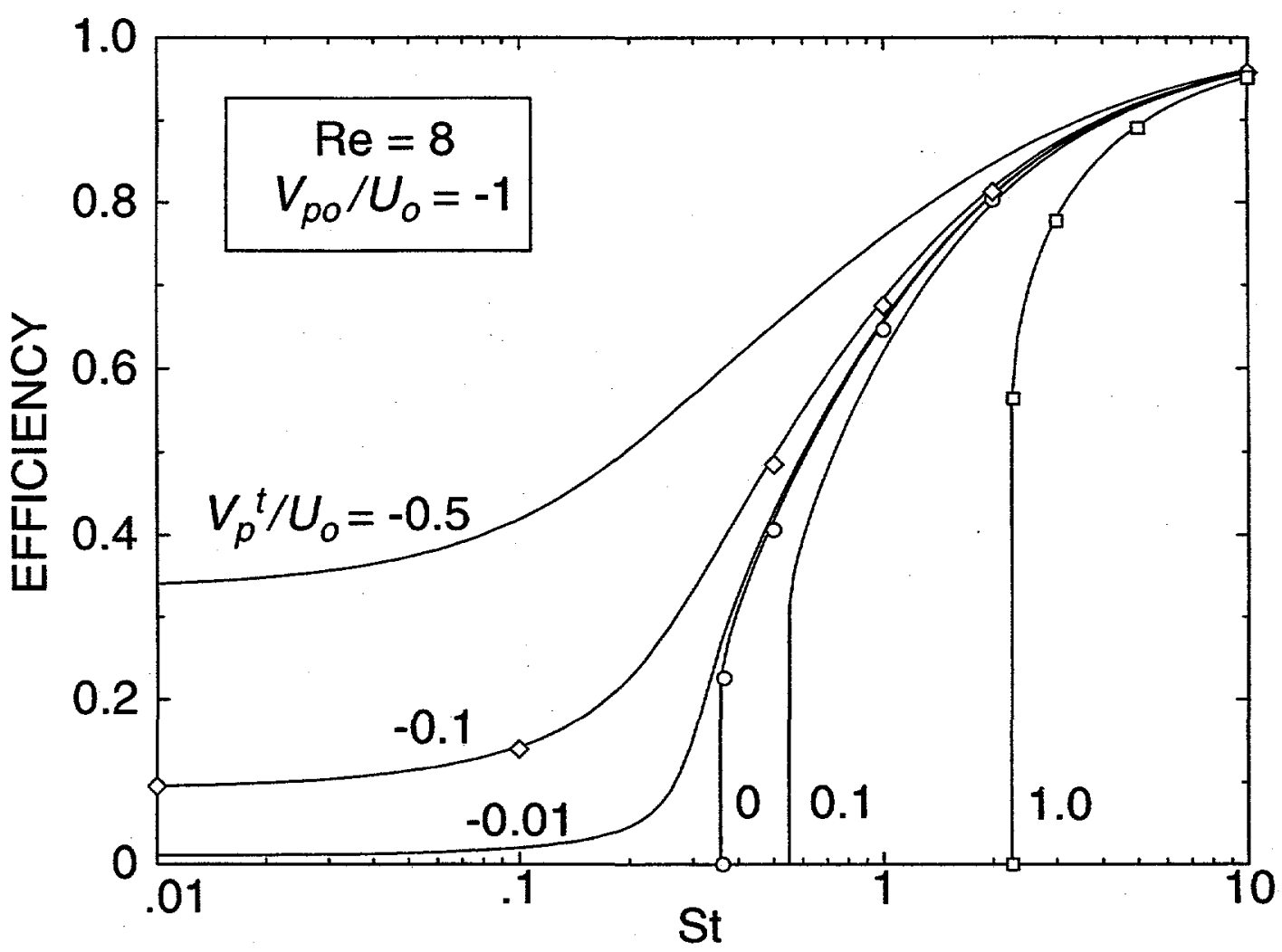

b)

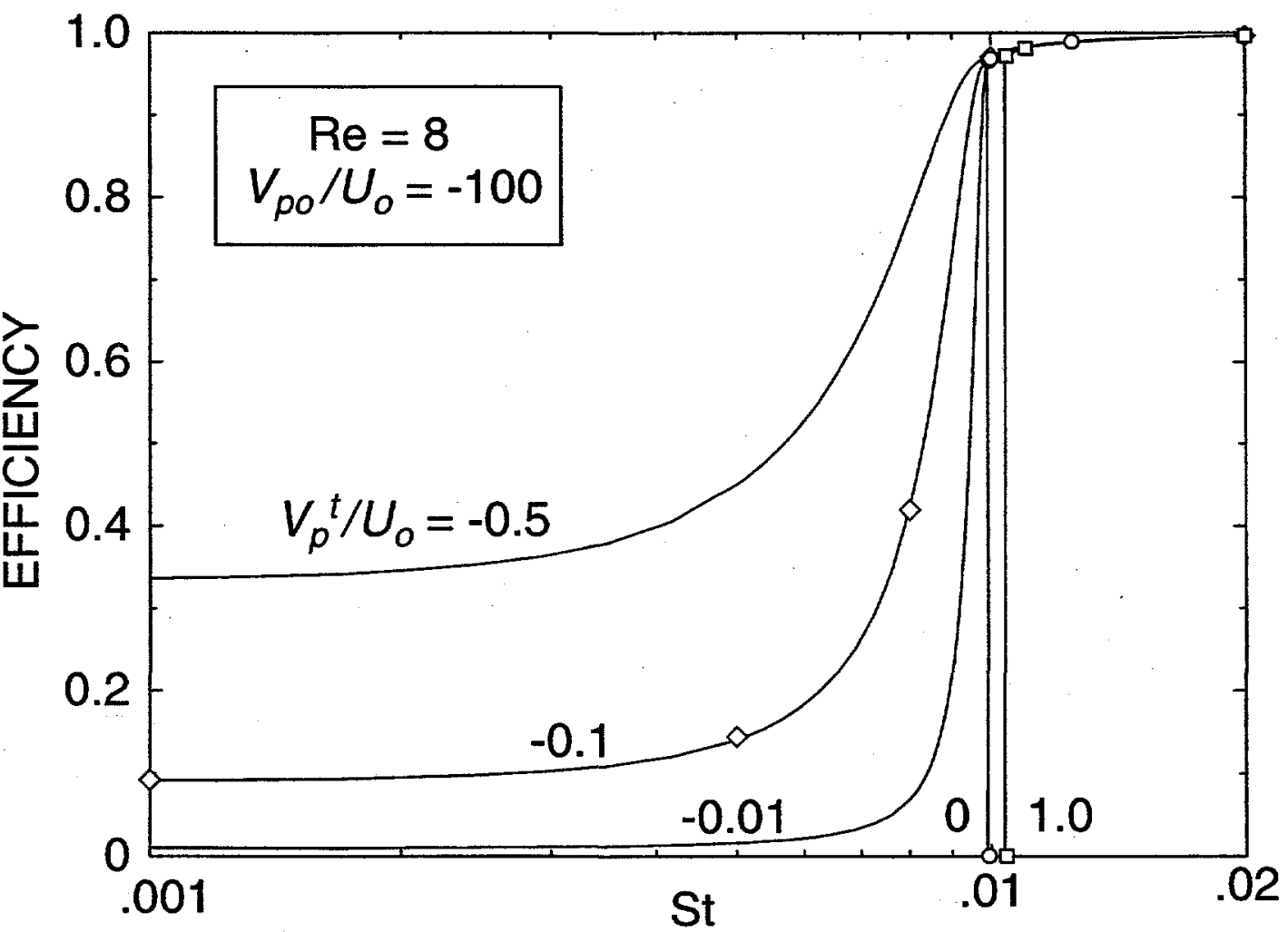

Figure 20. Efficiency vs. Stokes number for Various Drift Velocities for $R e=8$. Solid lines are calculated using an analytic flow field and a Runge-Kutta integrator, while the symbols are calculated using numerical flow solutions and the FIDAP particle tracking post-processing routines. a) Particle dimensionless initial velocity of $-1, b)$ Particle dimensionless initial velocity of -100 . 
ticular, particles entering the domain with large initial velocities would be expected to be fairly insensitive to the details of the flow field near the showerhead; this trend is observed in Figure $20 \mathrm{~b}$ for which the analytic and FIDAP results are nearly indistinguishable. Both the analytic and FIDAP results approach the low-St limit given by Equation (4.2) for $V_{p}^{t} / U_{o}<0$. That is, Equation (4.2) holds for non-diffusing, non-inertial particles ${ }^{3}$ entrained in any flowfield that conserves fluid mass (e.g., satisfies Equation (3.1)). The solutions calculated with FIDAP conserve mass within numerical accuracy, and it is readily shown that Equation (3.5) conserves mass exactly. At the other extreme, both techniques yield efficiencies approaching unity in the large Stokes-number limit.

For a more quantitative comparison, representative values for $\mathrm{St}_{\text {crit }}$ and the corresponding efficiency for both flow solution methods (analytic and numerical) are tabulated in Table 2 in the absence of external forces. The $\mathrm{St}_{\text {crit }}$ value was selected for these comparisons as its value is very sensitive to differences between flowfields. For a fixed particle initial velocity, the differences between the two flowsolution methods increase with Reynolds numbers; this effect is most pronounced at low initial particle velocities $\left(V_{p o} / U_{o}=-0.1\right.$ and -1) although even in these cases the differences in $\mathrm{St}_{c r i t}$ are less than

Table 2: Comparison of particle collection efficiencies calculated using analytic and FIDAP flowfields. Various combinations of particle initial velocities $V_{p o} / U_{o}$ and flow Reynolds numbers are presented for the case where no external force is acting $\left(V_{p}^{t} / U_{o}=0\right)$.

\begin{tabular}{|c|c|c|c|c|c|}
\hline \multirow[b]{2}{*}{$V_{p o} / U_{o}$} & \multirow[b]{2}{*}{$\operatorname{Re}$} & \multicolumn{2}{|c|}{ Analytic - Equation (3.5) } & \multicolumn{2}{|c|}{ FIDAP } \\
\hline & & $\mathrm{St}_{\text {crit }}$ & $\eta_{\text {crit }}$ & $\mathrm{St}_{\text {crit }}$ & $\eta_{\text {crit }}$ \\
\hline \multirow{4}{*}{-0.1} & 0 & 0.4753 & 0.1706 & 0.475 & 0.171 \\
\hline & 2 & 0.4488 & 0.1749 & 0.449 & 0.175 \\
\hline & 4 & 0.4247 & 0.1785 & 0.427 & 0.177 \\
\hline & 8 & 0.3832 & 0.1886 & 0.392 & 0.179 \\
\hline \multirow{4}{*}{-1} & 0 & 0.4210 & 0.2367 & 0.422 & 0.237 \\
\hline & 2 & 0.4037 & 0.2329 & 0.404 & 0.232 \\
\hline & 4 & 0.3873 & 0.2301 & 0.389 & 0.230 \\
\hline & 8 & 0.3571 & 0.2274 & 0.364 & 0.222 \\
\hline \multirow{4}{*}{-10} & 0 & 0.09186 & 0.7577 & 0.0919 & 0.758 \\
\hline & 2 & 0.09148 & 0.7485 & 0.0915 & 0.749 \\
\hline & 4 & 0.09109 & 0.7390 & 0.0912 & 0.742 \\
\hline & 8 & 0.09033 & 0.7218 & 0.0906 & 0.727 \\
\hline \multirow{4}{*}{-100} & 0 & 0.009917 & 0.9707 & 0.00992 & 0.971 \\
\hline & 2 & 0.009913 & 0.9693 & 0.00992 & 0.970 \\
\hline & 4 & 0.009909 & 0.9679 & 0.00991 & 0.968 \\
\hline & 8 & 0.009902 & 0.9653 & 0.00991 & 0.966 \\
\hline
\end{tabular}

\footnotetext{
3. For external forces that can be described by a potential, which includes both the gravitational and thermophoretic forces under the assumption of constant fluid properties.
} 
$2 \%$ and in efficiency are less than $5 \%$. In the case of creeping flow $(\mathrm{Re}=0)$, the analytic and numerical solutions for the flowfield are identical, so that the small discrepancies observed are the result of the less-accurate particle tracking capabilities of FIDAP compared to the Runge-Kutta formulation. ${ }^{4}$ For Reynolds numbers less than four, the use of the analytic flowfield provides a very accurate approximation of particle transport over a wide range of $V_{p o} / U_{o}$.

For a fixed Reynolds number, the differences between the two flow-solution methods decrease with increasing initial particle velocities. For large initial particle velocities (e.g., $\left.V_{p o} / U_{o}=-100\right)$, the critical Stokes numbers and efficiencies from the analytic and numerical schemes are in excellent agreement for all Reynolds numbers. This supports the claim made above: the details of the flowfield become less important for particles entering the domain at high velocity. Even for the worse case shown in Table 2, high Reynolds number $(\operatorname{Re}=8)$ and low particle initial velocity $\left(V_{p o} / U_{o}=-0.1\right)$, the differences in $\mathrm{St}_{\text {crit }}(<2 \%)$ and in efficiency $(<5 \%)$ are fairly modest. For low Reynolds numbers (say Re $<4$ ) and/or high initial velocities (say $\left|V_{p o} / U_{o}\right|>50$ ), the analytic approximation of is excellent.

The success of the analytic approximation for low Reynolds number flow greatly simplifies calculation of efficiencies, since the analytic result can be included in a robust Runge-Kutta integrator that runs quite efficiently on a workstation. Although numerical solutions of the flowfield are needed for more complicated 2- and 3-D geometries or for high Reynolds number flow, such programs are quite large and calculations require much more extensive computer resources (as well as a license). Unless otherwise indicated, all of the following work was performed using the analytic flow approximation.

\subsubsection{Effect of Fluid Reynolds Number}

The effect of Reynolds number on particle transport is expected to be greatest at low initial particle velocities; while for high particle initial velocities the influence of the flow field should be less important. Examples of these trends have already been presented in Table 2 neglecting external forces. At a low initial particle velocity of $V_{p o} / U_{o}=-0.1$ variations in $S t_{\text {crit }}$ were $24 \%$ between the $R e=0$ and 8 cases. At a high initial velocity of $V_{p o} / U_{o}=-100$ variations in $\mathrm{St}_{\text {crit }}$ were much smaller; e.g., less than a $0.2 \%$ difference when $R e$ is changed from zero to eight.

Additional calculations were performed with external forces acting; these results are plotted in Figure 21. The calculations were performed using the analytic approximation for the flow field for an initial particle velocity of $V_{p o} / U_{o}=-1$; this relatively low value was selected to accentuate any Reynoldsnumber effects. Figure 21 shows that the qualitative features of the efficiency curves do not change in the Reynolds number range of interest $(\operatorname{Re}<8)$. In particular, for a given external force (parameterized by $V_{p}^{t} / U_{o}$ ), the curves for all Reynolds numbers tend to the same large-Stokes limit (unity). For an attractive external force $\left(V_{p}^{t} / U_{o}<0\right)$, the curves for all Reynolds numbers tend to the same small-Stokes limit - Equation (4.2). However, a Reynolds number dependence is observed for intermediate Stokes numbers. In the absence of an external force or for a repulsive force $\left(V_{p}^{t} / U_{o} \geq 0\right)$, the value of $\mathrm{St}_{\text {crit }}$ does depend on Reynold number - although for a strong repulsive force this dependence is weak. Trajectory calculations (not shown) were also made for the same values of $V_{p}{ }^{t} / U_{o}$ and Re as used in Figure 21 but with a higher initial particle velocity of $V_{p o} / U_{o}=-100$; the resulting efficiency curves showed very little dependence on Re when $V_{p}{ }^{t} / U_{o} \geq 0$, though Reynolds number effects were still significant for attractive forces.

Although there are situations for which the particle collection efficiency is relatively insensitive to the flow field (e.g., for $V_{p}{ }^{t} / U_{o} \geq 0$ with $V_{p o} / U_{o}>>1$ ), in general the effect of Reynolds number should be included. This is not a serious obstacle, however, as the analytic flow field was shown in the previous section to work acceptably well for Reynolds numbers up to four for high aspect-ratio domains.

\footnotetext{
4. Based on these and other $\mathrm{Re}=0$ comparisons, the errors in FIDAP-calculated values for $\mathrm{St}$ crit and efficiency are less than $\sim 0.5 \%$.
} 


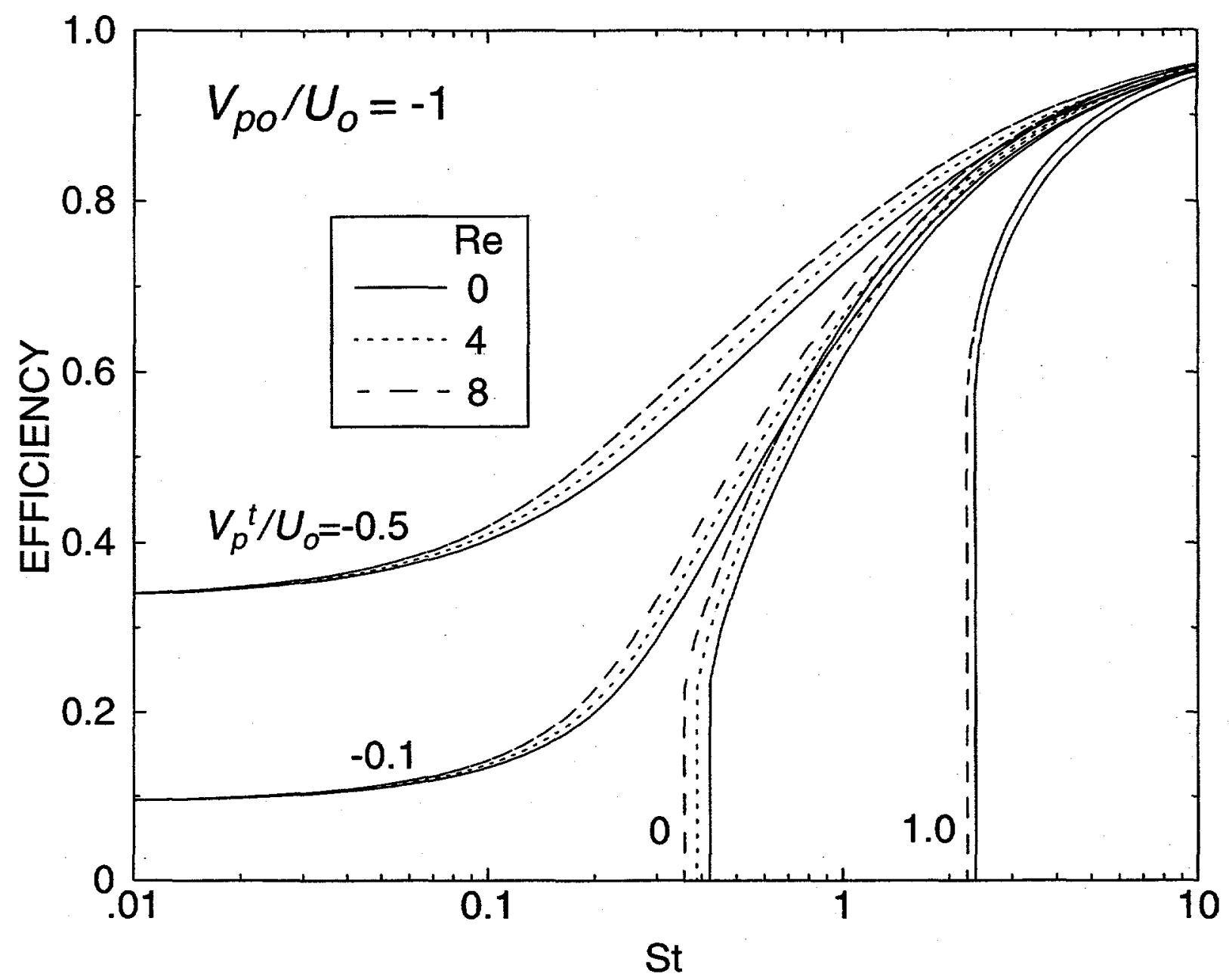

Figure 21. Efficiency vs. Stokes number for Various Drift Velocities and Reynolds Numbers. The initial particle velocity is -1 for all curves, which are calculated using the analytic flow field.

\subsubsection{Asymptotic Limit of Critical Stokes Number}

An interesting result is suggested by Figure $20 \mathrm{~b}$ and Table 2 for the case of no external force: as the particle initial velocity becomes large, the collection efficiency tends toward a step function which jumps from zero to unity at a critical Stokes value equal to the inverse of the dimensionless initial particle velocity. To confirm this result, a series of calculations were made to explore the dependence of the critical Stokes number on initial particle velocity in the absence of an external force. These results are shown in Figure 22, where $\mathrm{St}_{c r i t}$ is plotted as a function of $V_{p o} / U_{o}$ for fluid Reynolds numbers of 0,4 , and 8 . For a given value of $V_{p o} / U_{o}$, particles with $\mathrm{St}<\mathrm{St}_{c r i t}$ (below the line) will exit the reactor, while particles with $\mathrm{St}>\mathrm{St}_{\text {crit }}$ (above the line) will impact. The effect of Reynolds number is negligible for large values of $V_{p o} / U_{o}$; in fact, for $V_{p o} / U_{o}>10$ the three Re curves approach the same asymptotic limit. For large values of $V_{p o} / U_{o}$ particle inertia dominates deposition and the details of the flow field become unimportant. Inspection of the large initial-velocity asymptotic limit reveals the following relationship:

$$
\mathrm{St}_{\text {crit }} \rightarrow \frac{U_{o}}{V_{p o}}
$$




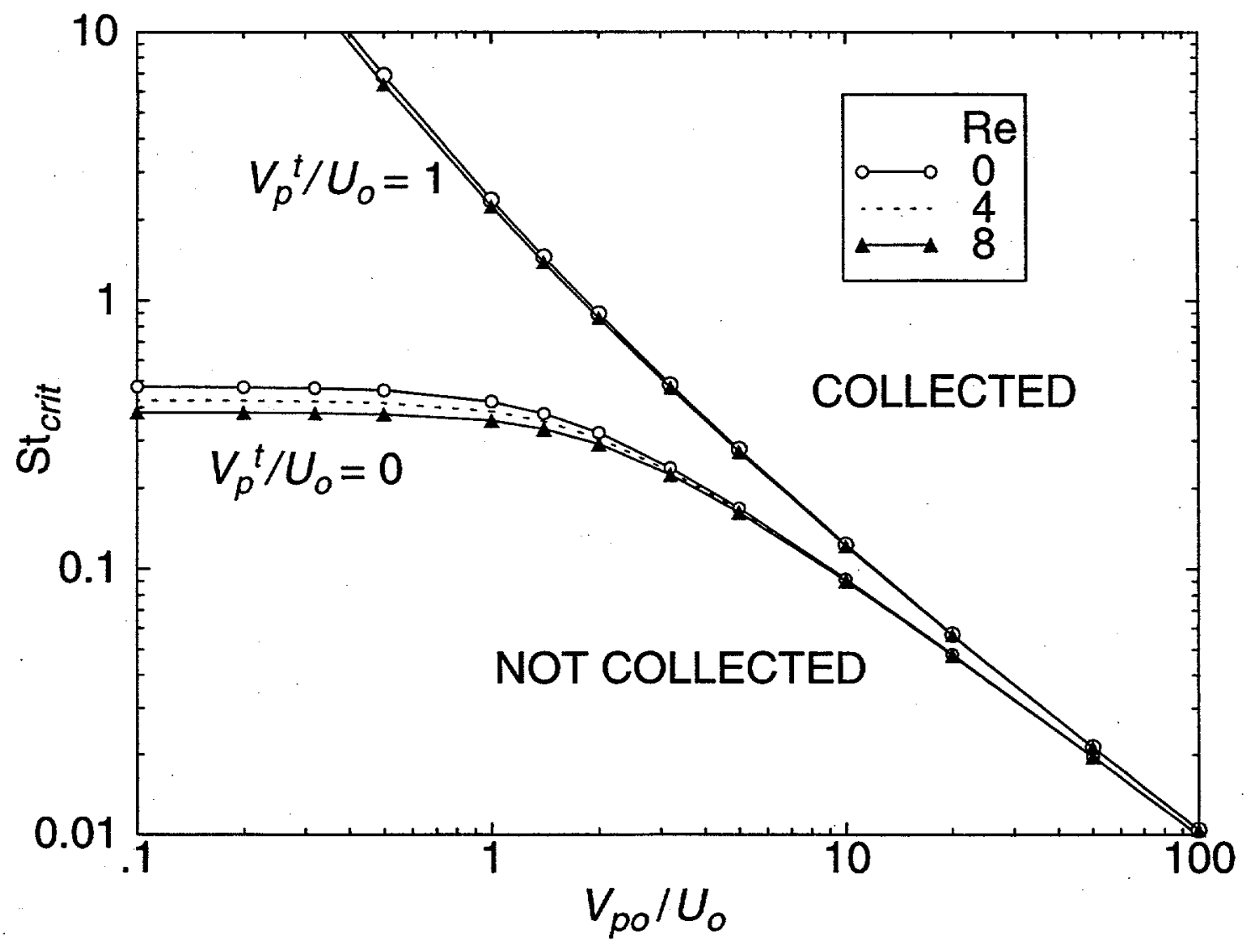

Figure 22. Critical Stokes Number vs. Particle Dimensionless Inlet Velocity. Values of the critical Stokes number were calculated using the analytic approximation to the flow field for Reynolds numbers of 0,4 , and 8 . One set of curves applies for no external force $\left(V_{p}^{t} / U_{o}=0\right)$, the other set applies for a strong force resisting deposition $\left(V_{p}^{t} / U o=1\right)$.

A simple explanation of this limit is readily illustrated by rearranging Equation (6.4) to give $\mathrm{St}_{c r i t} V_{p o} / U_{o}=\tau V_{p o} / S=1$, which states that impaction occurs when the particle stopping distance based on its initial velocity $V_{p o}$ equals the showerhead-to-wafer gap. ${ }^{5}$

Figure 22 also shows the variation of the critical Stokes number when a large external force opposing deposition is applied $\left(V_{p}^{t} / U_{o}=1\right)$. Although the value of $\mathrm{St}_{c r i t}$ is greatly increased for small $V_{p o} / U_{o}$ (compared to the case with no external force acting), all curves approach the same asymptote - Equation (6.4) - in the limit of very large initial particle velocity. The $\mathrm{St}_{\text {crit }}$ value for a large external force was found to be $\sim 4 \%$ higher than without an external force for $V_{p o} / U_{o}=100$. As noted above, the influence of Reynolds number on $\mathrm{St}_{c r i t}$ is greatly reduced when a resistive force is acting.

Thus, for no or repulsive external forces, a great simplification results for large values of the initial particle velocity (say for $V_{p o} / U_{o}>100$ ): the collection efficiency can be closely approximated by a step function (from zero to unity) at a critical Stokes number calculated by Equation (6.4). When an attractive force is present, the concept of a critical Stokes number breaks down, as there is some deposition at all Stokes numbers. Even in this case, however, a sharp increase in efficiency near $\mathrm{St}_{\text {crit }}$ is still seen (such as shown in Figure 20b).

5. A further implication of Equation (6.4) is that for large values of $V_{p d} U_{o}$ a more appropriate choice for the characteristic velocity in defining particle Stokes number would have been $V_{p o}$. 


\subsection{Coupled Transport - Nondimensional Results}

In this section, showerhead-enhanced inertial deposition is explored by coupling the transport of particles through the showerhead and in the inter-plate region. The procedure is as follows: 1) for given values of $U_{j e t} / U_{o}, L / S$, and St calculate the dimensionless velocity of the particle exiting the showerhead, $V_{p o} / U_{o}$, using Equations (6.1) and (6.2); and then 2) using $V_{p o} / U_{o}$ as the initial particle velocity, and the parameters $\mathrm{Re}, \mathrm{St}$, and $V_{p}{ }^{t} / U_{o}$, integrate the particle trajectory between the plates to determine the particle collection efficiency. Thus, the coupled particle transport problem (for an infinite parallel-plate geometry and under the present assumptions) is completely specified by five independent dimensionless parameters (note that $V_{p o} / U_{o}$ depends on the other four). Efficiency results from these coupled calculations should look qualitatively like those shown in Figures (20) and (21), although some variations are expected as the initial particle velocity is no longer fixed but depends on the degree of particle acceleration through the showerhead.

It is valuable at this point to clarify the use of the jet to face-velocity parameter $U_{j e t} / U_{o}$, which is the local fluid velocity that a particle experiences while passing through a showerhead hole. For the assumption of plug flow through the showerhead, $U_{j e t} / U_{o}=\bar{U}_{j e t} / U_{o}=A_{\text {showerhead }} / \Sigma A_{\text {jet }}$ where $\bar{U}_{\text {jet }}$ is the mean velocity in the tube. In the plug-flow case, $U_{j e t} / U_{o}$ must always be larger than unity and is constant across each showerhead tube cross-section. In commercial reactors, values of $\bar{U}_{j e t} / U_{o}$ are seldom less than twenty, and can range up to several hundred. The other limit considered in this work is fully-developed parabolic flow through the showerhead holes. In this case, $U_{j e t} / U_{o}$ is function of both the area ratio and the radial starting position of the particle in the showerhead tube. For example, a particle starting on the tube centerline would experience a fluid velocity twice the mean, so that $U_{j e t}(r=0) / U_{o}=2 \bar{U}_{j e t} / U_{o}$. Because the fluid velocity must vanish at the tube wall, jet to face-velocity ratios less than one are possible for the parabolic case for particles starting near the wall. In the following, results are parameterized with the most general form $U_{j e t} / U_{o}$.

\subsubsection{Critical Stokes Numbers}

One set of coupled efficiency calculations is shown in Figure 23, which plots efficiency versus Stokes number for jet to face-velocity ratios of $0.1,1,10,100$, and 1000. For these calculations, the showerhead thickness was assumed equal to the plate gap $(L / S=1)$ for the case of $\operatorname{Re}=0$ and no external forces acting $\left(V_{p}^{t} / U_{o}=0\right)$. The critical Stokes number (the smallest $\mathrm{St}$ for which collection occurs) is found to decrease with increasing values of $U_{j e t} / U_{o}$. This result is not surprising: as $U_{j e t} / U_{o}$ increases the particle velocity at the showerhead exit $\left(V_{p o} / U_{o}\right)$ must also increase, and we have shown in Section 6.3.1 that increasing values of $V_{p o} / U_{o}$ lead to smaller values for $\mathrm{St}_{\text {crit }}$ (see Figure 22). In particular, we have shown in the limit of large $V_{p o} / U_{o}$ that $\mathrm{St}_{c r i t} \rightarrow U_{o} / V_{p o}$. It is interesting to note that, for coupled transport, the large $U_{\text {jet }} / U_{o}$ limit of $\mathrm{St}_{\text {crit }}$ is not $U_{o} / U_{\text {jet }}$, but a slightly higher value (e.g., for $U_{j e t} /$ $U_{o}=100, \mathrm{St}_{c r i t}=0.01237$ ). This difference is explained by the fact that, because of inertia, the particle can't accelerate to the jet velocity before exiting the showerhead (i.e., $V_{p o} / U_{o} \leq U_{j e t} / U_{o}$ ); consequently, a larger Stokes number is required to initiate deposition. In the slow-jet limit, say $U_{j e t} / U_{o}<1$, the critical Stokes number becomes less sensitive to the particle inlet velocity, although the shapes of the efficiency curves can be quite different (e.g., compare the curves for $U_{j e t} / U_{o}=0.1$ and 1 ). Here, the details of the efficiency curve result from a complicated interplay among the parameters. Overall, reducing the value of $U_{j e t} / U_{o}$ (more and/or larger showerhead holes) increases $\mathrm{St}_{\text {crit }}$ - with the favorable result of increasing the minimum size for which inertial effects lead to particle deposition on the wafer.

The showerhead thickness also plays a role in determining the magnitude of the critical Stokes number. The effect of showerhead thickness is presented in Figure 24, in which collection efficiency is plotted against St for $L / S=0.1,0.5,1$ and 2 (for $U_{j e t} / U_{o}=100, R e=0$, and $V_{p}^{t} / U_{o}=0$ ). For large $L / S$ values, there is sufficient time in the showerhead for the particle to accelerate to the jet velocity $\left(V_{p o} / U_{o} \rightarrow U_{j e t} / U_{o}\right)$, which by Equation (6.4) gives the asymptotic limit $\mathrm{St}_{\text {crit }} \rightarrow U_{o} / U_{j e t}$. As seen in Figure 24, this limit is approached for $L / S>2$. For very thin showerheads, the particles spend only a short time in the showerhead, and will exit the showerhead with a velocity much less than the jet ve- 


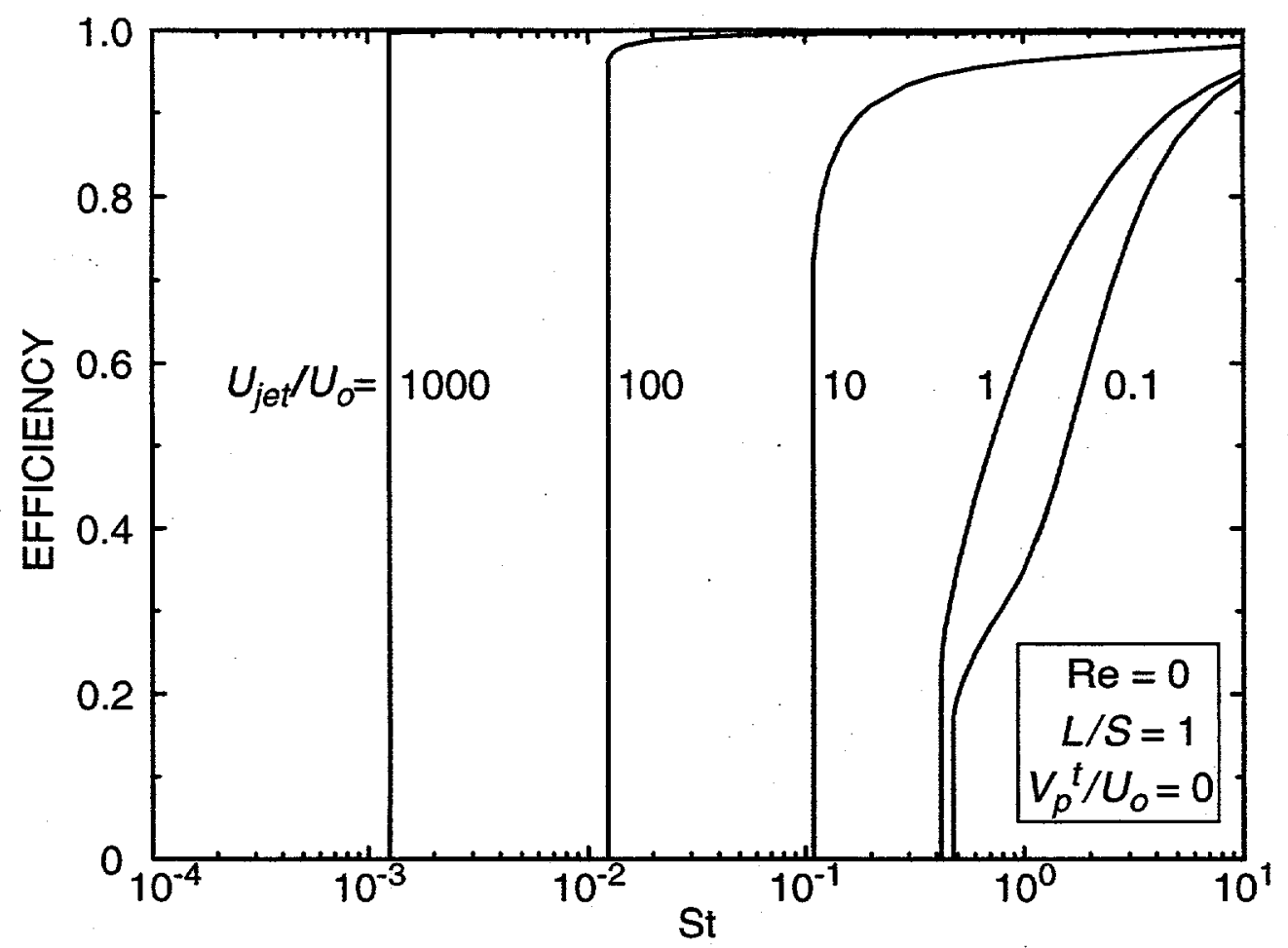

Figure 23. Effect of Jet to Face-Velocity Ratio. Efficiency versus Stokes number for $U_{j e t} / U_{o}=1,10,100$, and 1000 including coupling between showerhead and inter-plate transport (for this calculation $L / S=1, \operatorname{Re}=0, V_{p}{ }^{t} / U_{o}=0$, and $r_{p} / S=1 \times 10^{-7}$ ).

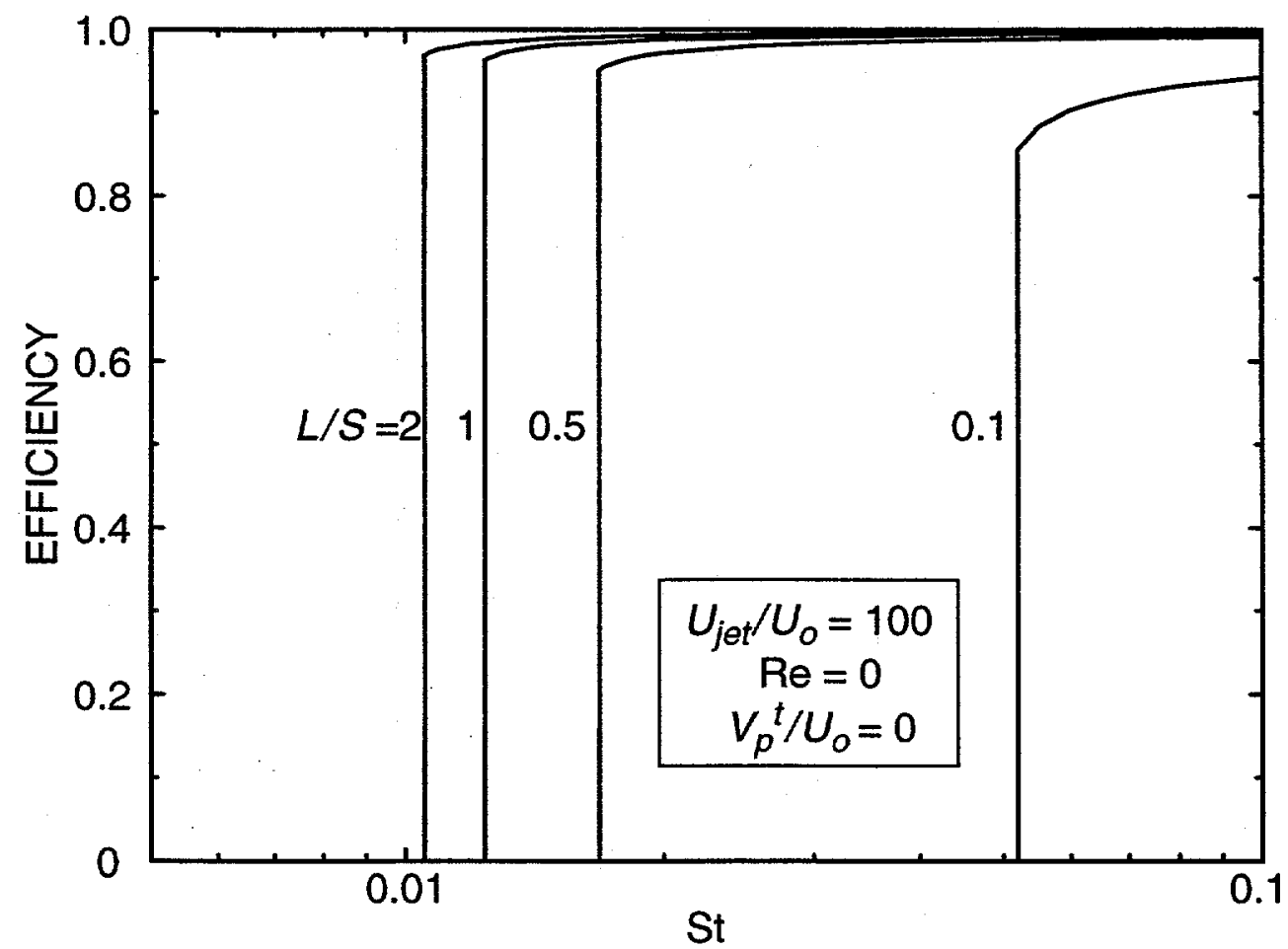

Figure 24. Effect of Showerhead Thickness. Efficiency versus Stokes number for $L / S=0.1,0.5,1$, and 2 including coupling between showerhead and inter-plate transport (for this calculation $U_{j e t} / U_{o}=100, \operatorname{Re}=0, V_{p}^{t} / U_{o}=0$, and $r_{p} / S=1 \times 10^{-7}$ ). 


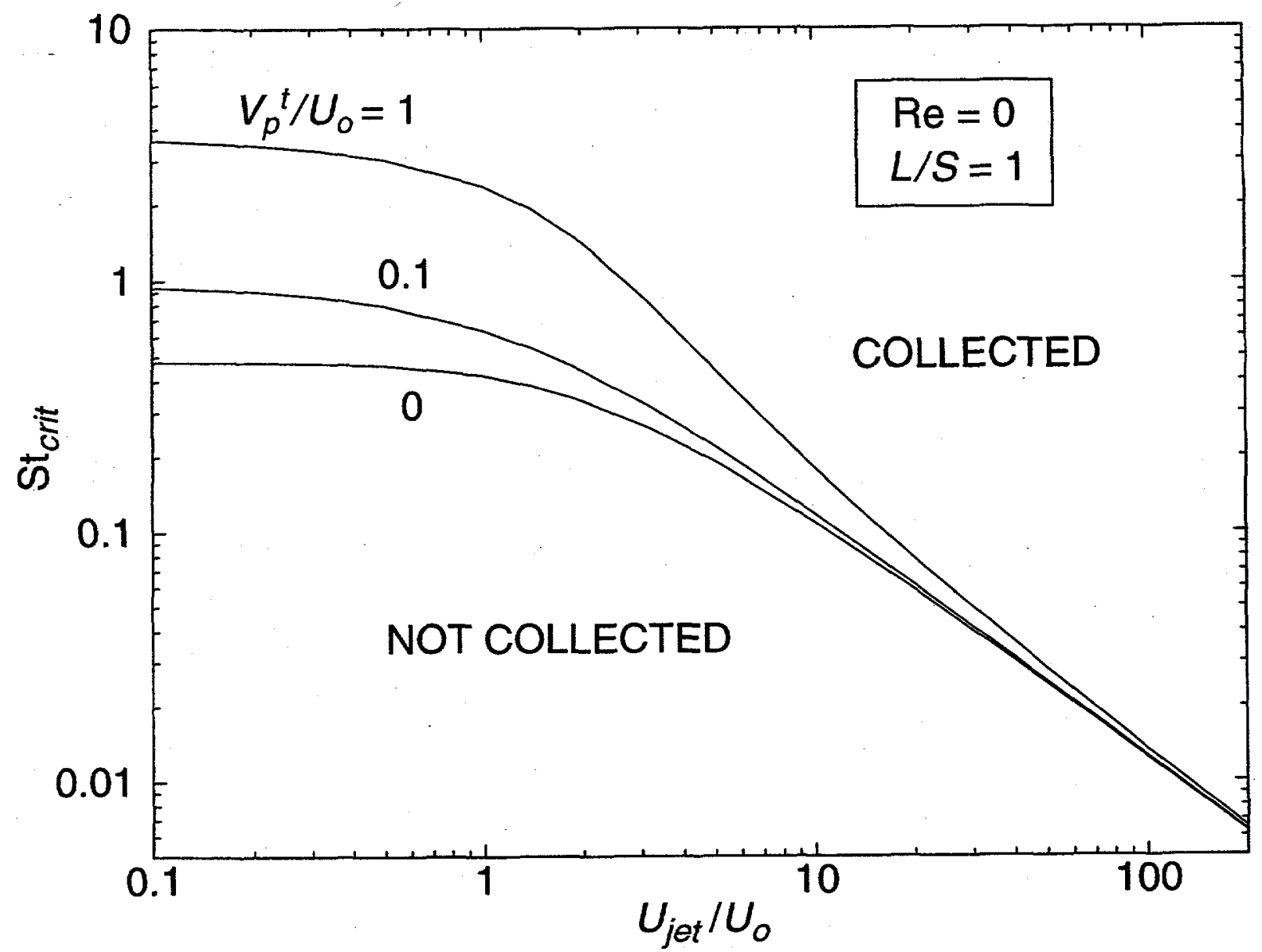

Figure 25. Critical Stokes Number vs. Jet to Face-Velocity Ratio. Values of the critical Stokes number were calculated vs. jet to face-velocity ratios for various levels of resistive forces $\left(V_{p}^{t} / U_{o}=0\right.$, 0.1 , and 1) including coupling between showerhead and inter-plate transport (for this calculation $L / S=1, \operatorname{Re}=0$, and $r_{p} / S=1 \times 10^{-7}$ ).

locity. In this case, larger Stokes numbers are needed to initiate deposition - as seen in Figure 24 for the curves with $L / S=0.1$ and 0.5 . Based on these results, $\mathrm{St}_{\text {crit }}$ can be increased (and inertial deposition reduced) by reducing the dimensionless showerhead thickness $L / S$.

An additional set of calculations was performed to determine the variation of critical Stokes number with different resisting forces $\left(V_{p}^{t} / U_{o} \geq 0\right)$ when the coupling between the showerhead and inter-plate regimes is considered. Some of these calculations are shown in Figure 25, where $\mathrm{St}_{\text {crit }}$ is plotted as a function of the jet to face-velocity ratio is plotted for the case of no external force acting, $V_{p}{ }^{t} / U_{o}=0$, and for repulsive forces, $V_{p}^{t} / U_{o}=0.1$ and 1 (assuming $\operatorname{Re}=0$ and $L / S=1$ ). ${ }^{6}$ Although $\mathrm{St}_{\text {crit }}$ is sensitive to the magnitude of the resisting force for low velocity ratios, for the large $U_{j e t} / U_{o}$ values typical of commercial reactors (say $U_{j e t} / U_{o}>20$ ) it is not. Thus, although a repulsive force will always help to reduce inertial deposition, the benefit will be small in systems characterized by large $U_{\text {jet }} / U_{o}$.

6. The value of $V_{p}^{t} / U_{o}=1$ corresponds to very strong repulsive forces which is unlikely to be exceeded in commercial reactors. 


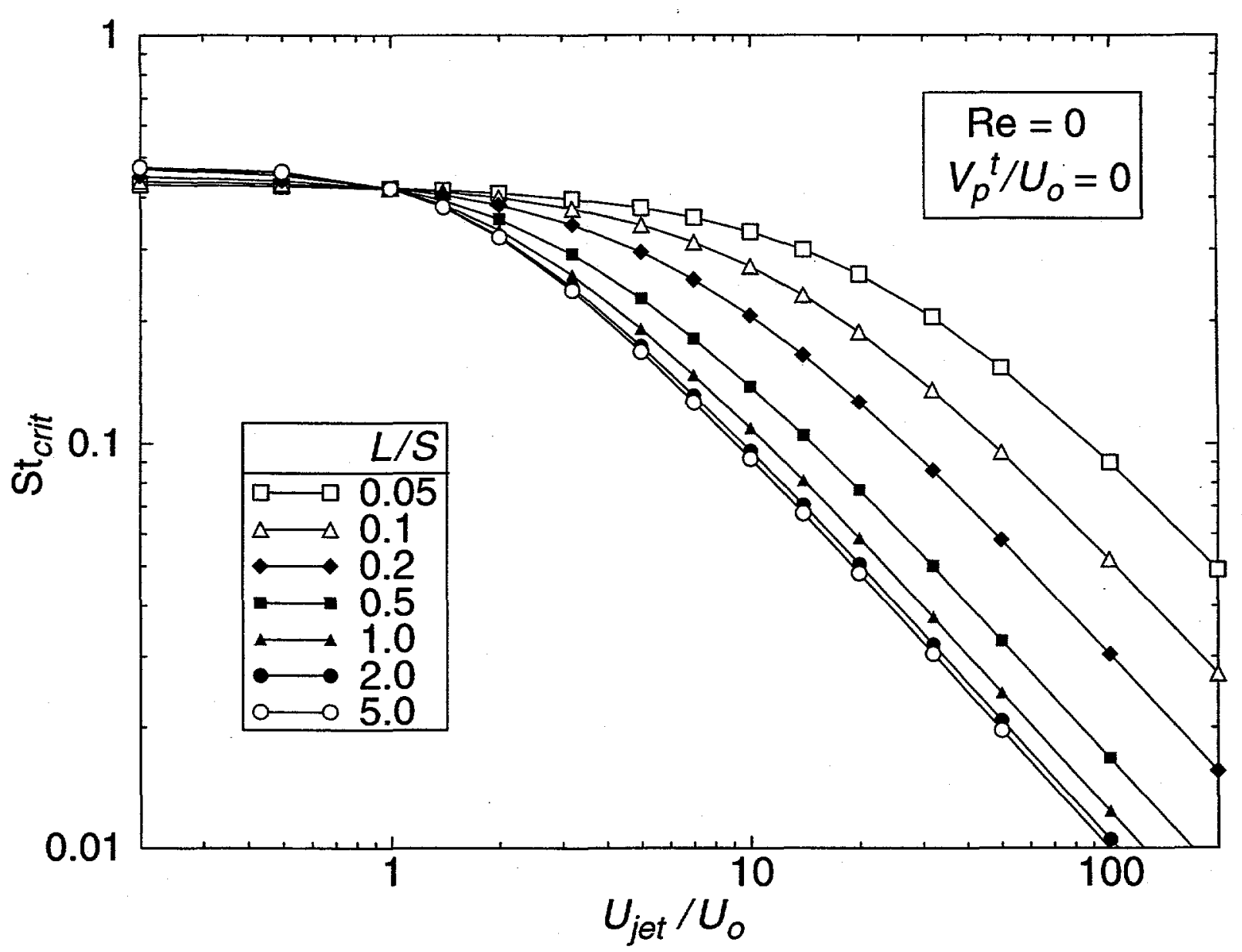

Figure 26. Grand Design Curve. Grand design curve for estimating the critical Stokes number based on showerhead parameters (for this calculation $\mathrm{Re}=0, V_{p}^{t} / U_{o}=0$, and $r_{p} / S=1 \times 10^{-7}$ ).

\subsubsection{Grand Design Curves}

If the effect of a repulsive force is neglected, the value of $\mathrm{St}_{\text {crit }}$ (for a given flowfield) is determined solely by the values of $U_{j e t} / U_{o}$ and $L / S$. Interestingly, both of these parameters are geometrical in nature. The geometric interpretation of $L / S$ is obvious (the ratio of showerhead thickness to inter-plate gap), while that for $U_{j e t} / U_{o}$ requires some explanation. Under the assumption of plug flow within the showerhead holes, it has already been shown that $U_{j e t} / U_{o}=\bar{U}_{j e t} / U_{o}=A_{\text {showerhead }} / \Sigma A_{\text {jet }}$ where $\bar{U}_{j e t}$ is the mean velocity in the tube. Thus, in the plug-flow limit the velocity ratio is completely specified by the number and size of the showerhead holes and by the diameter of the showerhead - purely geometric properties of the showerhead. Under the assumption of parabolic flow, the radial starting position of the particle within the showerhead hole must also be considered, but this is another a geometrical parameter. Thus, for a specific flowfield and neglecting external forces, the critical Stokes number is uniquely specified by chamber and showerhead geometry (and possibly an assumed particle starting position), and is independent of process parameters (e.g., gas temperature, pressure, or flow rate).

This simplification leads to the idea of the grand design curves, which give critical Stokes number as a function of the velocity ratio $U_{j e t} / U_{o}$ for various dimensionless showerhead thicknesses $L / S$. An example of a grand design curve over a wide range of these two parameters is shown in Figure 26 for 
$\operatorname{Re}=0$ and $V_{p}{ }^{t} / U_{o}=0$ (these values are also tabulated in Table 3 in the Appendix). The qualitative trends are consistent with earlier discussion: the critical Stokes number decreases with increasing values of $U_{j e t} / U_{o}$ and $L / S$. The curves for all values of $L / S$ intersect at $U_{j e t} / U_{o}=1$, which corresponds to the case for which the particle enters (and exits) the showerhead at the same velocity. Variations in $\mathrm{St}_{\text {crit }}$ with $L / S$ become small for dimensionless showerhead thicknesses larger than about two; in this case, the particle has had sufficient time to accelerate to near the gas velocity, so that making the showerhead longer has little effect. For velocity ratios less than one, the critical Stokes number is essentially independent of velocity ratio and the dimensionless showerhead thickness. For large velocity ratios, the curves in Figure 26 for different $L / S$ values become parallel with a slope of unity - suggest-

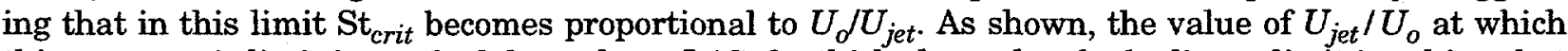
this asymptotic limit is reached depends on $L / S$ : for thick showerheads the linear limit is achieved at much smaller velocity ratios than for thin showerheads.

For design applications, the grand design curves are used with the parameters $U_{j e t} / U_{o}$ and $L / S$ to find the critical Stokes number for the proposed reactor geometry. To minimize particle deposition on the wafer, it is desirable to choose parameters that give as large a critical Stokes number as possible, as increasing $\mathrm{St}_{\text {crit }}$ increases the minimum size at which inertial deposition begins. Based on Figure 26, larger values of $\mathrm{St}_{\text {crit }}$ are obtained by decreasing $U_{j e t} / U_{o}$ (use more and/or larger showerhead holes) or by decreasing $L / S$ (use a thin showerhead or a large inter-plate gap). Using a resistive force (such as by heating the wafer relative to the showerhead) will always help, but as discussed above, the effect is fairly small under realistic conditions. Once $\mathrm{St}_{\text {crit }}$ has been determined, the corresponding particle critical size, $d_{p, c r i t}$, can be found from Equation (2.21). Note that although the critical Stokes number depends only on geometric parameters, the critical particle diameter depends on geometric, process, and particle parameters. Thus, particle density and gas pressure, temperature, type, and flow rate all play a role in determining $d_{p, \text { crit }}$. The effects of process parameters on showerhead-enhanced inertial deposition are discussed in a later section.

\subsubsection{External Forces}

Although particle deposition only occurs for $\mathrm{St}>\mathrm{St}$ crit for resistive external forces, deposition will always take place when attractive forces are present. To demonstrate this behavior, particle collection efficiencies calculated using fully coupled particle transport (i.e, including showerhead acceleration) are shown for various values of the external force in Figure 27 (for $\operatorname{Re}=0, U_{j e t} / U_{o}=100, L / S=1$ ). The results are very similar to those in Figure 20b, which gives efficiency for a fixed particle inlet velocity $\left(V_{p o} / U_{o}=100\right)$ instead of the present case where the showerhead exit velocity is calculated based on showerhead parameters. ${ }^{7}$ The large and small Stokes limits are the same: for small St inertial effects vanish and the efficiency must tend to Equation (4.2), while for large St inertia dominates and efficiency must tend to unity. At intermediate values of St there are some differences. For example, it is seen that the critical Stokes number for the coupled analysis is slightly larger than for the case where $V_{p o} / U_{o}$ is held constant; as discussed above, for a finite-length showerhead the particle velocity at the showerhead exit must be slightly less that $U_{j e t} / U_{o}$, so that a larger Stokes number is needed to initiate inertial deposition. Note that the case of constant $V_{p}^{t} / U_{o}$ (i.e., $V_{p}{ }^{t} / U_{o}$ is independent of particle size or Stokes number) corresponds to the physically meaningful situation in which a thermophoretic force is acting (as the thermophoretic drift velocity is independent of particle diameter in the free molecular regime). For other external forces - such as gravity - the net drift velocity will not be a constant but will vary with particle diameter (and hence on St).

\subsubsection{Parabolic Profile}

For fully developed parabolic flow in the showerhead hole, the gas velocity varies with radial position

\footnotetext{
${ }^{7}$ Note that although $\mathrm{Re}=8$ in Figure $20 \mathrm{~b}$ and $\mathrm{Re}=0$ in Figure 27, Reynolds number effects are small.
} 


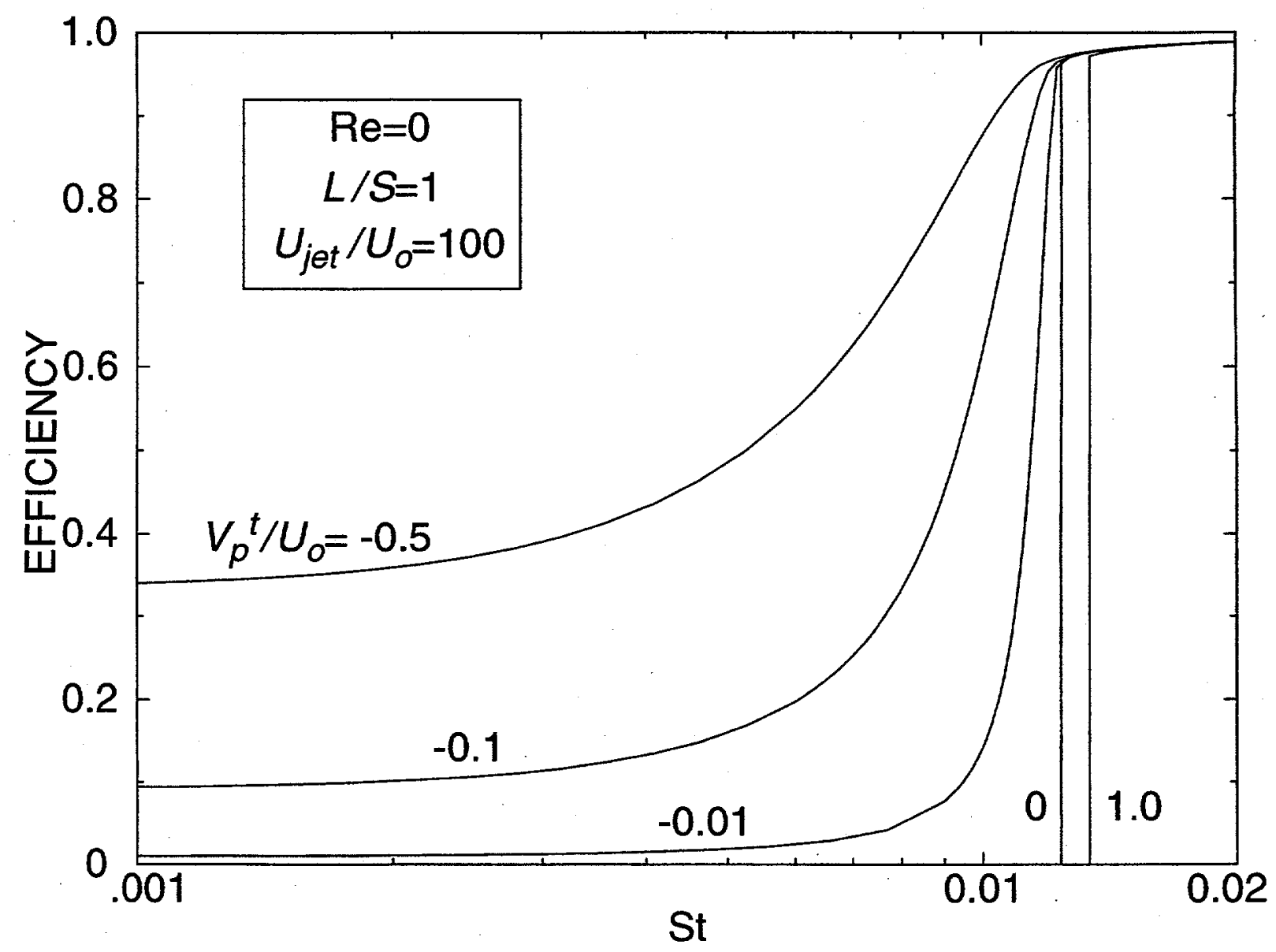

Figure 27. Efficiency vs. Stokes for various drift velocities. Collection efficiencies for fully coupled particle transport for $V_{p}^{t} / U_{o}$ values of $-0.5,-0.1,-0.01,0$, and 1 (for this calculation $\operatorname{Re}=0, L / S=1$, and $U_{j e t} / U_{o}=100$, and $r_{p} / S=1 \times 10^{-7}$ ).

within the hole as given by Equation (3.4); in this case the local velocity experienced by a particle in a showerhead hole depends on its radial starting position and the mean velocity $\bar{U}_{\text {jet }}$ (as given by Equation (3.3)). For example, a particle starting on the hole centerline will experience the highest local gas velocity $\left(2 \bar{U}_{j e t}\right)$, while ones starting near the hole wall will experience much lower velocities. As the amount of acceleration the particle experiences within the showerhead depends on the local gas velocity, the particle collection efficiency must vary with particle radial position within the showerhead hole. Thus, the calculation of the net collection efficiency for the parabolic flow case requires integrating the local efficiency radially across the showerhead hole. Assuming that the particles are uniformly distributed across the showerhead hole (i.e., that the flux of particles across the tube crosssection is constant), the net efficiency is:

$$
\eta_{n e t}(\mathrm{St})=\frac{2}{R_{j e t}^{2}} \int_{0}^{R_{j e t}} \eta\left[U_{j e t}(r), \mathrm{St}\right] r d r .
$$

The numerical integration of Equation (6.5) is computationally expensive, as each evaluation of the integrand $\eta\left[U_{j e t}(r)\right.$, St $]$ requires a coupled calculation of the particle acceleration through the showerhead (with local gas velocity $U_{j e t}(r)$ ) along with the corresponding numerical integration of the particle trajectory between the two plates. The integration of Equation (6.5) is further complicated by the 


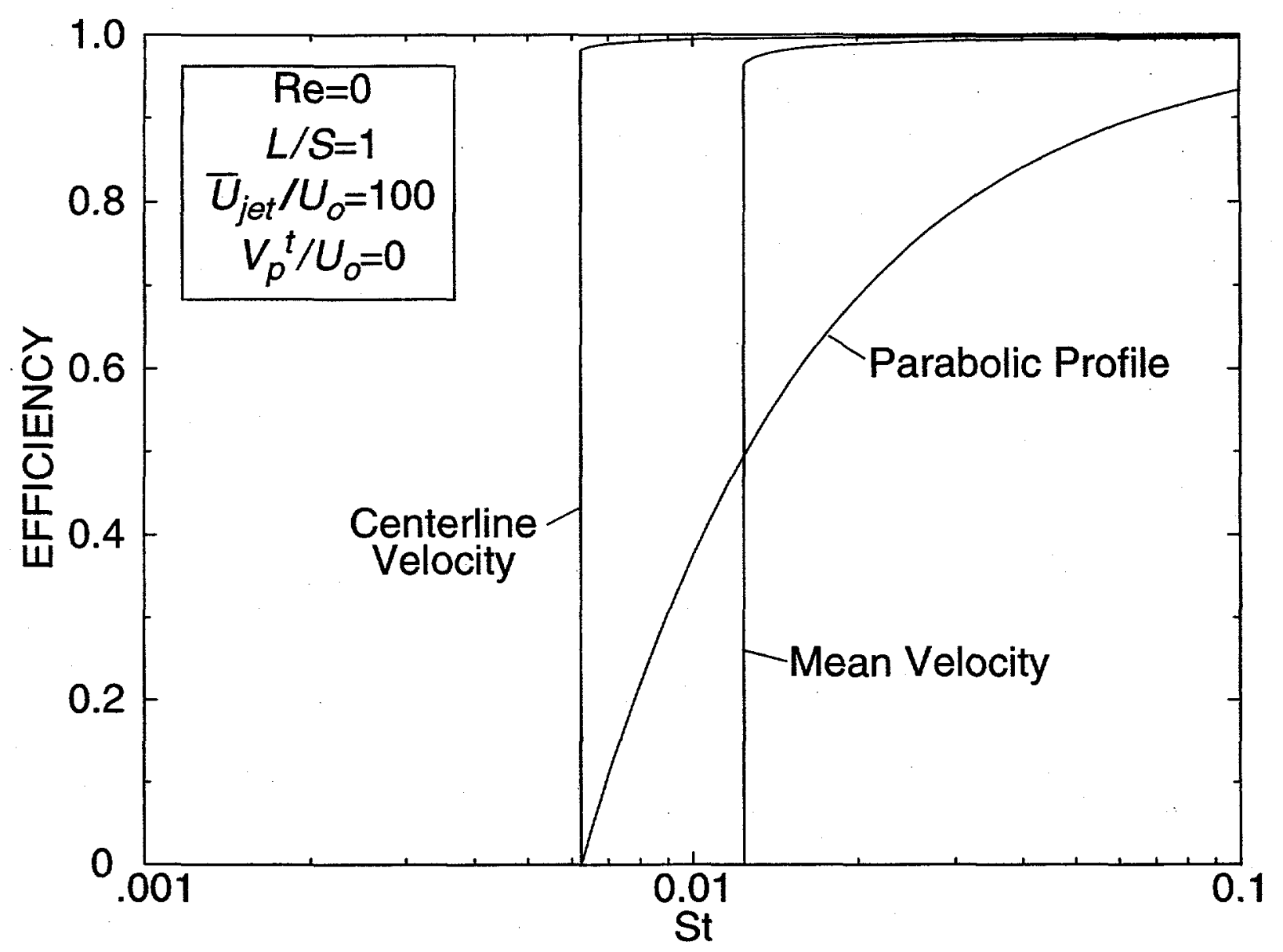

Figure 28. Efficiency vs. Stokes for parabolic showerhead profile (no external force). Collection efficiencies for fully coupled particle transport for particles experiencing the showerhead hole centerline and mean velocities (plug flow assumption), and integrated over the parabolic velocity profile in the showerhead holes in the absence of external forces $(\operatorname{Re}=0, L / S=1$, $V_{p}{ }^{t} / U_{o}=0$, and $\bar{U}_{j e t} / U_{o}=100$, and $r_{p} / S=1 \times 10^{-7}$ ).

fact that, in some cases, efficiency can change significantly with small changes in St or $U_{j e t}(r)$. In the present work an adaptive Gauss integration scheme with automatic error control has been used to evaluate Equation (6.5).

An example of a net efficiency curve for a parabolic velocity profile is shown in Figure 28 for the case where there is no external force ( $\operatorname{Re}=0, \bar{U}_{j e t} / U_{o}=100, L / S=1$, and $V_{p}^{t} / U_{o}=0$ ). For comparison, efficiency curves are also shown for plug flow (where all the particles experience the mean velocity) and for the hypothetical case where all of the particles experience the centerline velocity (essentially a plug flow moving at twice the mean velocity). These three curves show three interesting effects. First, the critical Stokes number for the centerline case is approximately one half that for the plug flow case; this result is explained by the fact that the critical Stokes number is inversely proportional to jet velocity in the limit of large values of $U_{j e t} / U_{o}$ (i.e., doubling the local showerhead gas velocity halves $\mathrm{St}_{\text {crit }}$, see Figure 25). ${ }^{8}$ Second, the efficiency curve for the parabolic case approaches unity more slowly than either of the plug flow curves. This is expected, as particles located near the tube wall experience

8. The critical Stokes number becomes less dependent on the velocity ratio for small $U_{\text {jet }} / U_{o}$. 
very low local velocities; for some region very close to the wall particles actually deaccelerate while passing through the showerhead. Note that although this slow approach to complete collection is favorable from a defect reduction point of view, it is not of practical significance because in this large Stokes regime the majority of particles entrained in the flow would still be deposited.

Third, the critical Stokes number for the parabolic case is the same as for the centerline case. The smallest particles deposited are those experiencing the highest velocity in the showerhead hole; thus, for parabolic flow inertia-enhanced deposition begins with those particles moving along the showerhead-hole centerline. The slope of the parabolic case is not as steep as for the centerline case because few particles are contained in the small-area region near the centerline, whereas in the centerline case we have assumed all of the particles are moving at the centerline velocity. The centerline case therefore serves as a limit of the smallest Stokes value for which inertial-enhancement to deposition becomes important in parabolic flow. In fact, the centerline case serves as a lower limit for all laminar flow conditions in the tubes, since even for developing flow the maximum velocity in the tube will always be less than $2 \bar{U}_{j e t}$. Thus, the most conservative practice for predicting the effects of inertia-enhanced deposition in real reactors is to use $U_{j e t} / U_{o}=2 \bar{U}_{j e t} / U_{o}$ as the characteristic velocity ratio in the grand design curves.

Another example of a net efficiency curve for a parabolic velocity profile is shown in Figure 29; in this case there is a relatively strong force acting to increase particle deposition $\left(\operatorname{Re}=0, \bar{U}_{j e t} / U_{0}=100\right.$, $L / S=1$, and $V_{p}{ }^{t} / U_{o}=-0.1$ ). Although the general trends are much the same as in Figure 28, the efficiency curve for the parabolic case now lies closer to the mean velocity case than to the centerline velocity case. Although the centerline case still provides a lower bound to inertial-enhancement, for strong attractive forces it is far too conservative. This result is explained by considering that, in the presence of a strong external force, particles starting very near the centerline are deposited whether inertia is important or not. Thus, inertial effects only become noticeable somewhere away from the centerline in a region better characterized by the mean velocity.

The present results show that, for parabolic flow in the showerhead, the best practice for determining net efficiency is to perform the full integration of Equation (6.5). However, since this calculation can be computationally expensive, the following approximations are suggested: for attractive external forces (say $V_{p}^{t} / U_{o}<-0.01$ ) use the mean velocity approximation, otherwise (say $V_{p}^{t} / U_{o}>0$ ) use the more conservative centerline approximation.

\subsection{Coupled Transport - Dimensional Results}

The two approaches to reduce inertia-enhanced particle deposition are: ${ }^{9} 1$ ) design equipment with as large a value of $\mathrm{St}_{c r i t}$ as possible, and 2) select process conditions that give as high a value of $d_{p, c r i t}$ as possible. Based on our previous analysis, the only three ways to increase $\mathrm{St}_{\text {crit }}$ is to design for minimum $\bar{U}_{j e t} / U_{o}$ and/or $L / S$, and to apply an external force that resists deposition. To minimize $\bar{U}_{j e t} / U_{o}$, very porous showerhead designs are needed to reduce the constriction of the flow (decrease the area ratio given by Equation (3.3) by either increasing the number or size of holes). The ratio $L / S$ can be reduced by reducing the showerhead thickness or by increasing the showerhead-to-wafer gap. Intuitively, a short showerhead thickness reduces the time available to accelerate the particle, and a large showerhead-to-wafer gap provides the particle more opportunity to slow down. Finally, a resistive external force could be used to inhibit inertial deposition such as by keeping the wafer warmer than the showerhead to take advantage of thermophoresis (remember that the resistive force typically had a fairly weak effect on reducing inertia-enhanced deposition).

Once a hardware design is fixed, $\mathrm{St}_{\text {crit }}$ is fixed, but it is still possible to minimize inertial deposition by selecting process conditions that give as high a value of $d_{p, c r i t}$ as possible. Based on the free molecular definition of Stokes number in Equation (2.23), we can see that low face velocities $U_{o}$ and large show-

9. It is impossible to eliminate inertial effects, as one can always imagine a particle large enough so that it will impact. 


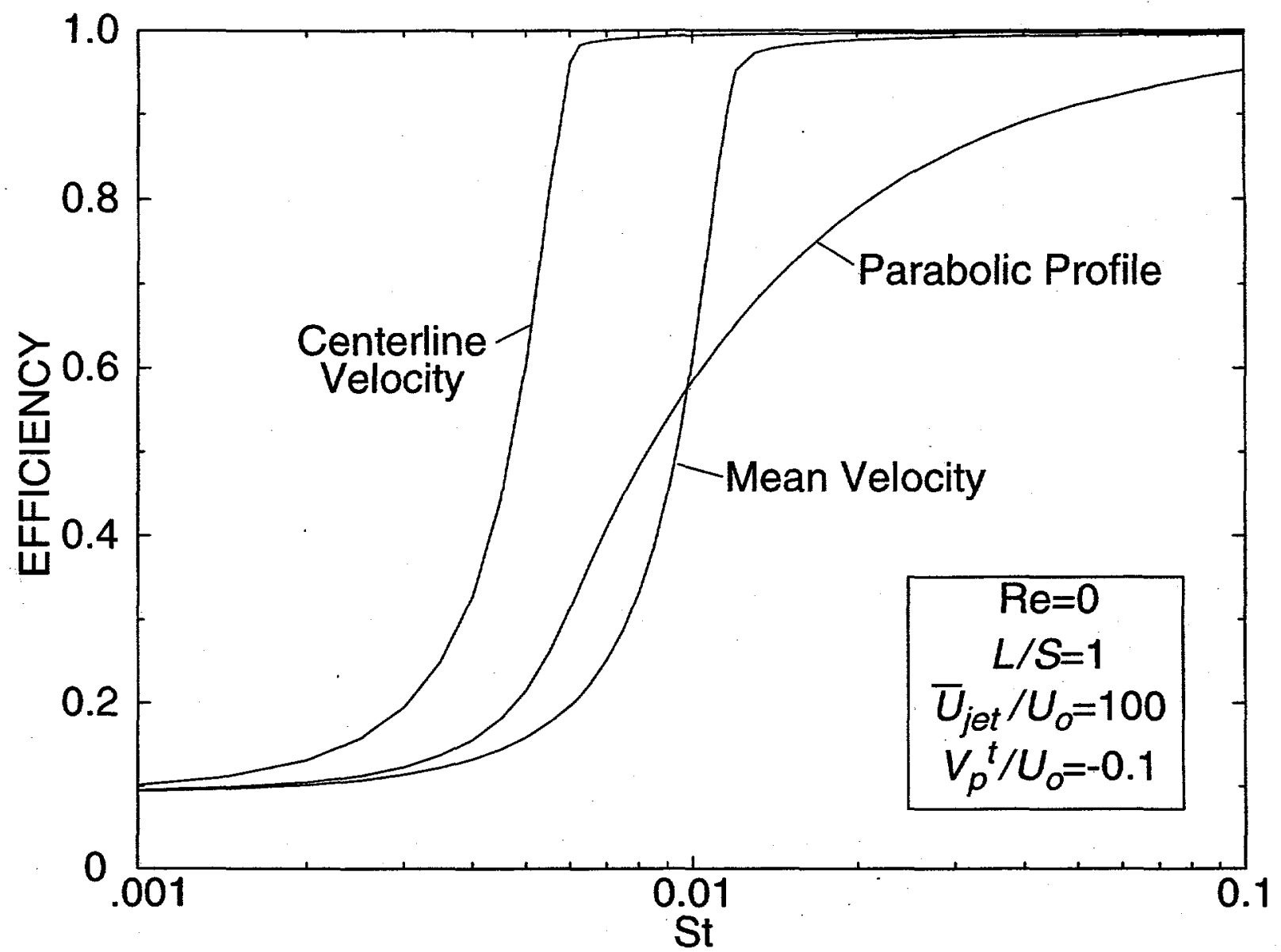

Figure 29. Efficiency vs. Stokes for parabolic showerhead profile (attractive force). Collection efficiencies for fully coupled particle transport for particles experiencing the showerhead hole centerline and mean velocities (plug flow assumption), and integrated over the parabolic velocity profile in the showerhead holes for the case of a attractive force (Re $=0, L / S=1, V_{p}{ }^{t} / U_{o}=-0.1$, and $\bar{U}_{j e t} / U_{o}=100$, and $\left.r_{p} / S=1 \times 10^{-7}\right)$.

erhead-to-wafer gaps $S$ are preferred. Mean gas velocities can be reduced by reducing mass flow rates (at constant pressure) or by operating at higher pressures (for a fixed mass flow rate). As seen in Equation (2.23), operating at higher pressures also directly increases $d_{p, c r i t}$. Thus, for a constant mass How rate, a twofold increase in pressure produces a fourfold increase in $d_{p, \text { crit }}$ (one factor of two directly from the pressure reduction, and an additional factor of two from lowering the face velocity) ${ }^{10} \mathrm{Re}-$ call that Equation (2.23) strictly applies to particles in the free molecular limit (small sizes and/or low pressures) which is a reasonable assumption of the pressure and particle size regimes in typical semiconductor manufacturing reactors.

An example of particle collection efficiency as a function of particle size is shown in Figure 30 for a hypothetical $200 \mathrm{~mm}$ (diameter) reactor characterized by: an argon flow rate of $1000 \mathrm{sccm}$ (standard cubic centimeters per minute) through a showerhead $2.54 \mathrm{~cm}$ thick with 1000 holes of diameter 0.0635

\footnotetext{
10. For higher pressures and/or larger particle diameters the quadratic relationship between pressure and critical diameter fails as the free-molecule expression for Stokes number given by Equation (2.23) becomes inaccurate. In the continuum limit the particle relaxation time becomes independent of pressure and proportional to diameter squared, so that a fourfold pressure increase would result in a twofold increase in $d_{p, c r i t}$. Thus, the influence of pressure on $d_{p, c r i t}$ is most pronounced in the free-molecule regime.
} 


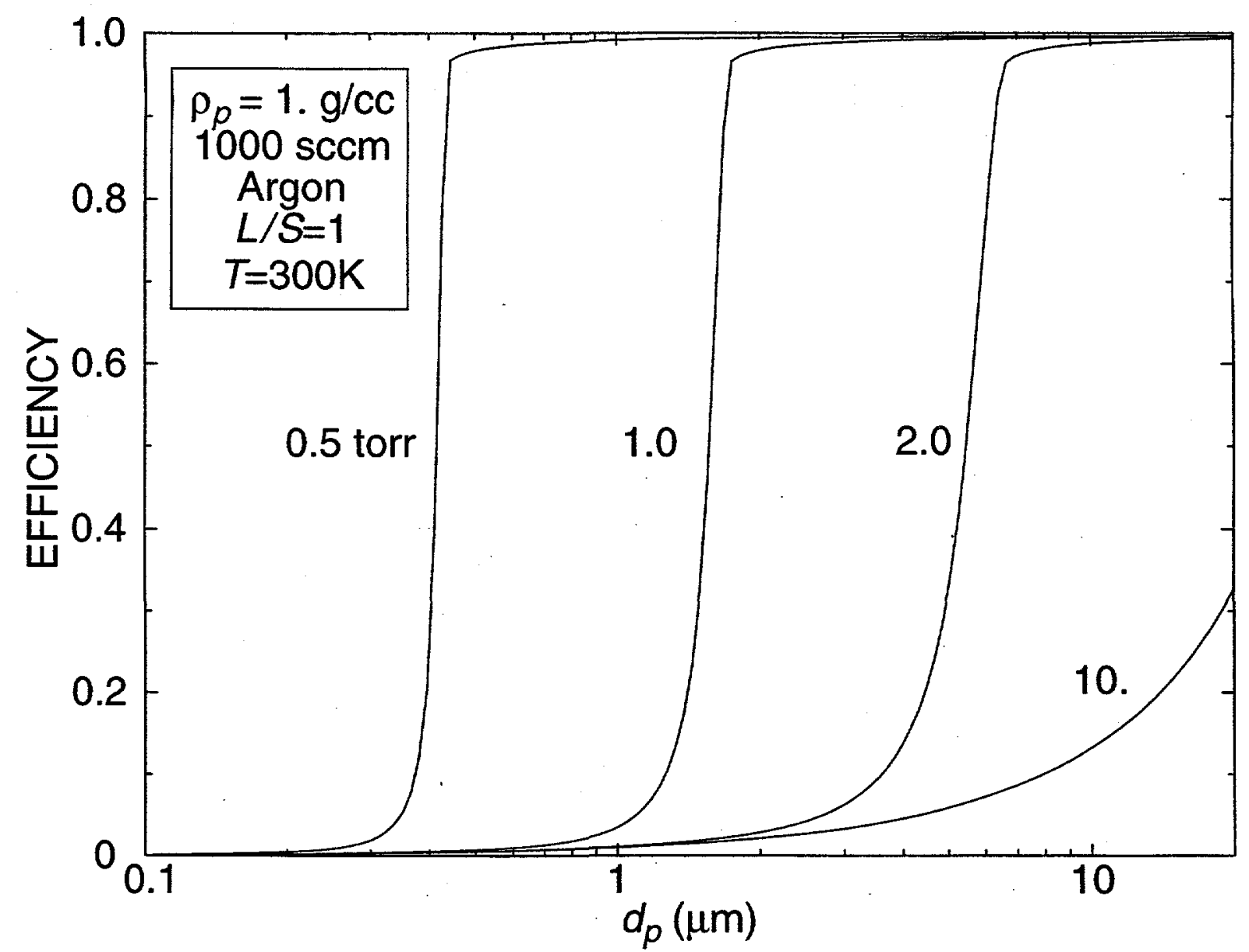

Figure 30. Efficiency us. particle diameter and pressure (isothermal case). Collection efficiencies for fully coupled particle transport assuming plug flow through the showerhead hole for reactor pressures of $0.5,1.0,2.0$, and 10 . torr $\left(\operatorname{Re}=0.98, L=S=2.54 \mathrm{~cm}, \bar{U}_{j e t} / U_{o}=99.2, Q=1000 \mathrm{sccm}\right.$ argon, $T=300 \mathrm{~K}$, and particle density of $1 \mathrm{~g} / \mathrm{cc}$ ).

$\mathrm{cm}$ with a showerhead-to-wafer gap of $2.54 \mathrm{~cm}$. The flow is assumed isothermal and a particle density of $1 \mathrm{~g} / \mathrm{cc}$ used. These reasonably physical parameters give the dimensionless quantities $L / S=1$, $\bar{U}_{j e t} / U_{o}=99.2$, and $\mathrm{Re}=0.984$; the dimensionless drift velocity varies with size according to the expression for gravitational settling velocity - Equation (2.26). Efficiency curves are shown for reactor pressures (i.e., pressure between the two plates) of $0.5,1.0,2.0$, and 10. torr. For pressures less than 2 torr, inertial-enhancement to deposition is clearly evident by the abrupt jump in efficiency from nearly zero to unity in the vicinity of a critical size. As seen, this critical size is a strong function of pressure, with an approximately fourfold decrease in the critical size for a twofold decrease in chamber pressure. For chamber pressures above about 10 torr inertial effects no longer contribute to deposition; the increase in efficiency for increasing particle size seen in Figure 30 for the $P=10$ torr case results because the gravitational drift velocity increases with size.

The same reactor geometry and process conditions have been used to calculate the effect of thermophoresis on collection efficiency as shown in Figure 31. The showerhead temperature has been held constant at $300 \mathrm{~K}$ while the wafer (lower plate) temperature is made colder $(280 \mathrm{~K})$, isothermal $(300 \mathrm{~K})$, or hotter (320K). Fairly modest temperature differences have been used in accordance with our isothermal (constant gas properties) assumption for flow calculations, but those small temperature differences are allowed to drive particle thermophoresis. The isothermal case shows that, for small particle sizes, deposition decreases with decreasing size because the gravitational drift velocity is de- 


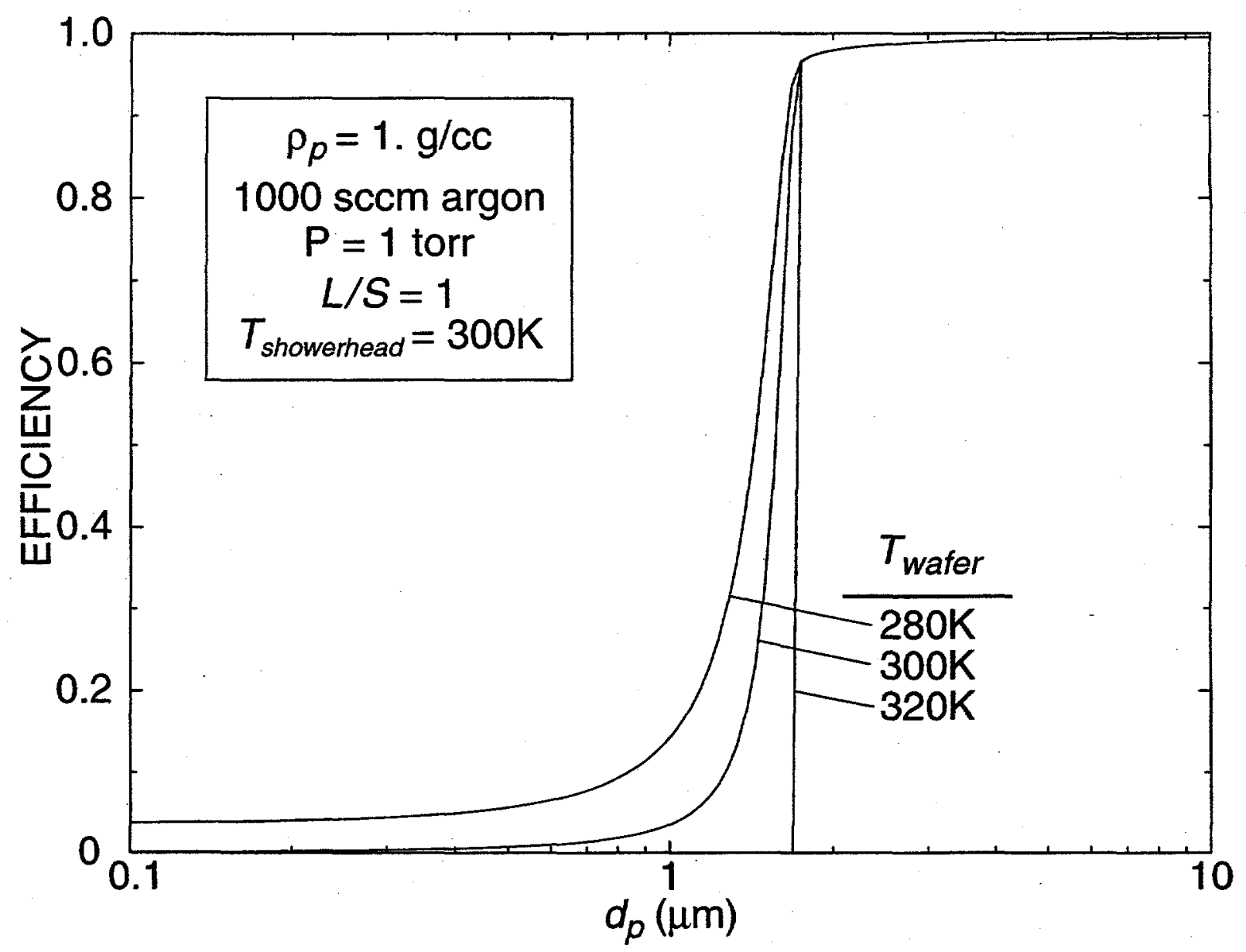

Figure 31. Efficiency us. particle diameter and wafer temperature (with thermophoresis). Collection efficiencies for fully coupled particle transport assuming plug flow through the showerhead hole for wafer temperatures of 280,300 , and $320 \mathrm{~K}$ ( $\operatorname{Re}=0.98, L=S=2.54 \mathrm{~cm}, \bar{U}_{\text {jet }} / U_{o}=99.2$, $Q=1000 \mathrm{sccm}$ argon, $P=1$ torr, $T_{\text {showerhead }}=300 \mathrm{~K}$, and particle density of $1 \mathrm{~g} / \mathrm{cc}$ ).

creasing. When the wafer temperature is less than the showerhead temperature, thermophoresis acts to increase deposition compared to the isothermal case. Although the differences become small for sizes larger than $d_{p, c r i t}$ (about $1.5 \mu \mathrm{m}$ ), the thermophoretic contribution is clear in the small-particle limit where the efficiency approaches a constant. ${ }^{11}$ Heating the wafer relative to the showerhead eliminates all small-particle deposition as the thermophoretic resistive force overwhelms the attractive gravitational force. The critical diameter at which inertial effects dominate is shifted to a slightly larger size than for the isothermal case, although the shift is fairly small since the effect of an exter$\mathrm{nal}$ force on $\mathrm{St}_{\text {crit }}$ is fairly weak. By using thermophoretic protection and by designing a reactor with a sufficiently large $d_{p \text {,crit }}$ particle deposition can be mitigated over the particle size range of interest.

The two previous examples highlight two important points: 1) that inertia-enhanced deposition becomes dramatically more important at low pressure, and 2) that thermophoretic protection coupled with careful control of inertial effects can significantly reduce particle deposition. Dimensional calculations could also be used to demonstrate all of the effects reported in Section 6.3, however, because of the wide variability in reactor geometry and particle and process parameters, only these two examples are presented.

\footnotetext{
11. Since the thermophoretic drift velocity is independent of particle diameter (Equation (2.31)) while the settling velocity is proportional to diameter, for small particles thermophoretic deposition dominates and the efficiency becomes constant.
} 


\subsection{Summary}

This chapter explored showerhead-enhanced inertial deposition in a parallel plate geometry characteristic of a wide range of semiconductor process tools. One common feature of a showerhead design is that the area available to the flow is constricted inside the showerhead; consequently, the high velocity gas inside the showerhead hole can accelerate particles which pass through the showerhead. Depending on conditions, particle acceleration by the showerhead can lead to inertia-enhanced deposition on the wafer below. A Lagrangian (or particle tracking) formulation was used in this work, which is ideal for predicting particle transport resulting from the combination of applied external forces and particle inertia; the current implementation does not account for particle diffusion.

Numerical models were presented for determining the extent of inertia-enhanced deposition in a parallel-plate geometry for particles originating upstream of the showerhead. The problem was treated in two steps, in which particle and fluid transport were determined: 1) within a showerhead-hole, and 2) between the showerhead and wafer. For flow in the showerhead, analytic expressions from Chapter 3 were used for two limiting cases: plug and fully-developed parabolic flow. Given the flow field, particle transport analysis led to an analytic expression for the dimensionless particle velocity at the exit of the showerhead, $V_{p o} / U_{o}$, as a function of two geometric parameters (the dimensionless jet velocity, $U_{j e t} / U_{o}$, and the ratio of the showerhead thickness to inter-plate gap, $\left.L / S\right)$ and the particle Stokes number, St.

The flow field in the inter-plate region was calculated analytically using a quasi-1-D asymptotic approximation, and numerically with a numerical finite-element simulation. For Reynolds numbers, Re, less than about four, the two methods were found to be in good agreement. A Lagrangian analysis was also used to describe particle transport in the inter-plate region to determine whether a particle entering the domain through the showerhead deposits on the wafer. The result of this analysis was a determination of the particle collection efficiency (fraction of particles entering the reactor that deposit on the wafer) as a function of $V_{p o} / U_{o}$, reactor geometry, Re, St, and the dimensionless particle drift velocity $\left(V_{p}^{t} / U_{o}\right.$ - a measure of the strength of applied external forces). For Reynolds numbers less than four, particle deposition efficiencies calculated with the analytic approximation for the interplate flow field agreed very well with efficiencies calculated using numerical flow solutions.

To calculate the net effect of inertia-enhanced deposition, the analysis of particle acceleration in the showerhead was combined with that for inter-plate transport. The final result was a determination of the particle collection efficiency as a function of $U_{j e t} / U_{o}, L / S, \operatorname{Re}, V_{p}^{t} / U_{o}$, and St. Results were presented in both nondimensional and dimensional forms. A key result was a calculated value for the critical Stokes number $\left(\mathrm{St}_{c r i t}\right)$, which is the smallest dimensionless particle size for which particles deposit (in the absence of any external forces or for a net external force acting to accelerate particles toward the wafer). The concept of a grand design curve was introduced, which gives $\mathrm{St}_{\text {crit }}$ as a function of the dimensionless jet velocity, $U_{j e t} / U_{o}$ for various showerhead thickness ratios, $L / S$. The focus of the discussion was to identify strategies that might be used to reduce particle deposition in commercial reactors.

Two approaches to reduce inertia-enhanced particle deposition were identified: 1) design equipment with as large a value of $\mathrm{St}_{\text {crit }}$ as possible, and 2) select process conditions that give as high a value of $d_{p, c r i t}$ as possible. Based on the present analysis, $\mathrm{St}_{\text {crit }}$ is determined primarily by reactor and showerhead geometry. Only three methods were identified for increasing $\mathrm{St}_{\text {crit }}$ :

- Decrease the showerhead velocity ratio $\bar{U}_{j e t} / U_{o}$ by increasing the number of and/or enlarging the size of the showerhead holes.

- Decrease the showerhead thickness ratio $L / S$ by making the showerhead very thin or the inter-plate gap large

- Apply an external force that resists deposition (as by keeping the wafer warmer than the adjacent gas). 
Given a specific hardware design (and corresponding $\mathrm{St}_{\text {crit }}$ ), inertial deposition can be reduced by selecting process conditions that give as high a value of $d_{p, c r i t}$ as possible. Based on the free molecule definition of Stokes number in Equation (2.23), the following general guidelines are offered to increase the critical diameter (for a given $\mathrm{St}_{\text {crit }}$ ):

- Increase the gap between the showerhead and wafer

- Use low mass flow rates

- Raise chamber pressure

- Use a high molecular weight gas.

All of the above recommendations specifically pertain to reducing particle inertial deposition that results from particle acceleration in the showerhead; note that these guidelines may conflict with those intended to reduce deposition by other mechanisms (e.g., gravity or diffusion). In order to reduce particle deposition in real tools, it is up to equipment designers/operators to identify the dominant deposition mechanism so that an effective improvement strategy can be identified. In addition, equipment designers should be aware that while these recommendations should improve particle performance, the effect of any changes on process performance must be investigated. 
BLANK PAGE 


\section{Summary}

This report presented numerical models for assessing particle transport and deposition in a parallelplate geometry, providing a computational tool for predicting the number of particles depositing on a wafer during semiconductor processing. No attempt was made here to analyze particle generation mechanisms; for the present discussion, particles were assumed to originate either: 1) upstream of the showerhead with a known concentration or 2) from a specified position between the plates with a fixed number or at a known generation rate. Thus, while the present transport analysis addressed the question of the fraction of gas-borme particles that deposit on the wafer, a prediction of the number of particles that deposit on the wafer additionally requires a clear understanding of the controlling particle generation mechanisms. In practical terms, the present analysis helps identify strategies for reducing the probability that particles are transported to and deposit on a wafer; a complete strategy for reduction of total particle-on-wafer counts also requires that particle source terms be understood and controlled

The basic phenomena controlling particle transport and the underlying general equations were reviewed in Chapter 2; note that the field of particle transport is quite broad so that for brevity discussion proceeded with an emphasis on conditions encountered in semiconductor process tools (i.e., subatmospheric pressures and submicron particles). Included in the discussion were expressions for the following particle forces: fluid drag, gravity, thermophoresis, and electric. The concepts of particle drift velocity and stopping distance were introduced, and issues of continuum vs. free molecular particle transport were outlined. Particle concentrations were assumed to be low enough to allow a dilute approximation, for which the coupling between the fluid and particle phases is one-way. In this case, the fluid/thermal transport equations can be solved either analytically or numerically neglecting the particle phase; the resulting velocity and temperature fields were then used as input for the particle transport calculations. In all of the present work isothermal flow was assumed, although small temperature differences were allowed to drive particle thermophoresis; both analytic and numerical solutions of the flow field were presented. Descriptions of the techniques used to calculate fluid flow in the parallel plate geometry were given in Chapter 3.

In order to provide a single parameter that can be used to compare particle deposition among many cases, Chapter 4 introduced a particle collection efficiency. The collection efficiency was defined as the fraction of particles present in the inter-plate region of the reactor that deposit on the wafer. Particles were presumed to either enter the reactor through the showerhead (uniformly spread between $r=0$ and $R_{W}$ ), or to originate in a plane parallel to the wafer. The latter case would correspond to particles being released from a plasma trap upon plasma extinction; in this case the particles are initially assumed to be uniformly spread radially between $r=0$ and $R_{W}$ at some distance $h$ from the wafer. Analytic expressions for collection efficiency were presented in Chapter 4 for the limiting case where external forces control deposition (i.e., neglecting particle diffusion and inertia).

Particle transport in the parallel-plate geometry was predicted using both the Lagrangian approach (where individual particle trajectories are calculated) and the Eulerian approach (where the particle concentration field is modeled as a continuum). The strength of the Eulerian formulation is in predicting particle transport resulting from the combination of applied external forces (including the fluid drag force) and the chaotic effect of particle Brownian motion (i.e., particle diffusion), although the current implementation cannot account for particle inertia. In particular, the Eulerian formulation cannot accommodate particle acceleration effects within the showerhead, and is therefore restricted to particle transport in the inter-plate region.

The Eulerian formulation, presented in Chapter 5, yielded an analytic, integral description of particle deposition for the case where the flow field between the plates can be approximated analytically. The need to properly account for diffusion-enhanced particle deposition becomes increasingly important as the semiconductor industry moves toward smaller feature sizes and becomes concerned with smallersized particles. Based on the Eulerian analysis, the following guidelines are intended to help tool operators and designers reduce particle deposition when diffusional effects are important: 
- Keep traps as far from wafer as possible

- Take advantage of repulsive forces, such as by thermophoretic protection gained by keeping the wafer warmer than the showerhead

- Reduce attractive forces

- For a specific pressure, use as high a mass flow rate as possible

The strength of the Lagrangian formulation was in predicting particle transport resulting from the combination of applied external forces and particle inertia, although the current implementation cannot account for particle diffusion. It is the Lagrangian formulation, treated in Chapter 6, that can properly account for inertia-enhanced deposition resulting from particle acceleration in the showerhead. The problem was treated in two steps, in which particle and fluid transport were determined: 1) within a showerhead-hole, and 2) between the showerhead and wafer. For fluid and particle transport in the showerhead, approximate analytic expressions can be derived based on a few assumptions. The output of this first step is the particle velocity at the exit of the showerhead, as a function of showerhead geometry, flowrate, and gas and particle properties. The particle showerhead-exit velocity was next used as an initial condition required for particle transport between the plates. The output of the second step is a determination of whether the particle deposits on the wafer, as a function of showerhead-exit particle velocity, the plate separation, flowrate, and gas and particle properties. Based on the Lagrangian analysis, two approaches were identified to help tool operators and designers reduce particle deposition when inertial effects are important: 1) design equipment with as large a value of $\mathrm{St}_{\text {crit }}$ as possible, and 2) select process conditions that give as high a value of $d_{p, c r i t}$ as possible. Based on this analysis, $\mathrm{St}_{\text {crit }}$ is determined primarily by reactor and showerhead geometry. Only three methods were identified for increasing $\mathrm{St}_{\text {crit }}$ :

- Decrease the showerhead velocity ratio $\bar{U}_{j e t} / U_{o}$ by increasing the number of and/or enlarging the size of the showerhead holes.

- Decrease the showerhead thickness ratio $L / S$ by making the showerhead very thin or the inter-plate gap large

- Apply an external force that resists deposition (as by keeping the wafer warmer than the adjacent gas).

Given a specific hardware design (and corresponding $\mathrm{St}_{\text {crit }}$ ), inertial deposition can be reduced by selecting process conditions that give as high a value of $d_{p ; c r i t}$ as possible. Based on the free molecule limit of Stokes number, the following general guidelines are offered to increase the critical diameter (for a given $\mathrm{St}_{\text {crit }}$ ):

- Increase the gap between the showerhead and wafer

- Use low mass flowrates

- Raise chamber pressure

- Use a high molecular weight gas.

The previous recommendations specifically pertain to reducing particle deposition given an assumed dominant deposition mechanism; note that one set of guidelines ( e.g., for inertia) may conflict with those intended to reduce deposition by other mechanisms (e.g., gravity or diffusion). In order to reduce particle deposition in real tools, it is up to equipment designers/operators to first identify the dominant deposition mechanism so that an effective improvement strategy can be identified. Note that the guidelines given above are not intended to replace detailed calculations (using the proper analysis with the actual process conditions), but to provide the user with a general feel for inherently-clean practices. In addition, equipment designers should be aware that while these recommendations should improve particle performance, the effect of any changes on process performance must be investigated. 


\section{References}

1. Allen, M.D. and Raabe, O.G. (1982) "Re-evaluation of Millikan's oil drop data for the motion of small particles in air," J. Aerosol Sci. 6: 537-547.

2. Bae, G.-N., Lee, C.S., and Park, S.O. (1994) "Measurement of particle deposition velocity toward a horizontal semiconductor wafer by using a wafer surface scanner," Aerosol Sci. Technol. 21: 7282.

3. Barnes, M. S., Keller, J. H., Forster, J. C., O’Neill, J. A., and Coultas, D. K. (1992) Phys. Rev. Lett. 68: 313.

4. Batchelor, G.K. and Shen, C. (1985) "Thermophoretic deposition of particles in gas flowing over cold surfaces," J. Colloid Interface Sci. 107(1): 21-37.

5. Bender, C.M. and Orszag, S.A. (1978) Advanced Mathematical Methods for Scientists and Engineers, McGraw-Hill, New York, p. 261.

6. Bird, R.B., Stewart, W.E., and Lightfoot, E.N. (1960) Transport Phenomena, Wiley and Sons, New York.

7. Broday, D., Fichman, M., Shapiro, M., and Gutfinger, C. (1997) "Motion of diffusionless particles in vertical stagnation flows - I. General model and deposition efficiency of spheres," J. Aerosol Sci. 28(1): 23-34.

8. Busnaina, A., Zheng, X., and Zhu, X. (1993) "Three-dimensional computer simulation of gas flow and particle transport in a horizontal diffusion furnace," J. Inst. Environ. Sci. 36(2): 25-32

9. Bowling, R.A. and Larrabee, G.B. (1989) "Behavior and detection of particles in vacuum processes,” J. Electrochem. Soc. 136(2): 497-502.

10. Chari, K. and Rajagopalan, R. (1985) “Deposition of colloidal particles in stagnation-point flow," $J$. Chem. Soc. Faraday Trans. 2 81: 1345-1366.

11. Choi, S.J., Rader, D.J., and Geller, A.S. (1996) "Massively parallel simulations of Brownian dynamics particle transport in low pressure parallel-plate reactors," J. Vac. Sci. Technol. A 14(2): 660-665.

12. Choi, S. J., Ventzek, P. L. G., Hoekstra, R. J. and Kushner, M. J. (1994) Plasma Sources Sci. Technol. 3: 418.

13. Clift, R., Grace, J.R., and Weber, M.E. (1978) Bubbles, Drops, and Particles, Academic Press, New York.

14. Collins, S., O’Hanlon, J.F., Carlile, R. and Kang, J. (1996) presented at Dusty Plasma-95 (Wickenburg, AZ, Oct. 1-7, 1995) (to appear in J. Vacuum Sci. Technol.).

15. Coltrin, M.E., Kee, R.J., Evans, G.H., Meeks, E., Rupley, F.M., and Grcar, J.F. (1991) "SPIN (Version 3.83): a Fortran program for modeling one-dimensional rotating-disk/stagnation-flow chemical vapor deposition reactors," SAND91-8003, Sandia National Laboratories, Albuquerque, New Mexico.

16. Cooper, D.W. (1986) "Particulate contamination and microelectronics manufacturing: an introduction," Aerosol Sci. Technol. 5: 287-299

17. Cooper, D.W., Miller, R.J., Wu, J.J., and Peters, M.H. (1990) "Deposition of submicron aerosol particles during integrated circuit manufacturing: theory," Part. Sci. Technol. 8: 209-224. 
18. Cooper, D.W., Peters, M.H., and Miller, R.J. (1989) "Predicted deposition of submicrometer particles due to diffusion and electrostatics in viscous axisymmetric stagnation-point flow," Aerosol Sci. Technol. 11: 133-143.

19. Cunningham, E. (1910) "On the velocity of steady fall of spherical particles through fluid medium," Proc. R. Soc. A-83, 357-365.

20. Dabros, T. and van de Ven, T.G.M. (1983) "A direct method for studying particle deposition onto solid surfaces," Colloid \& Polymer Sci. 261: 694-707.

21. Donovan, R.P., Yamamoto, T., Periasamy, R., and Clayton, A.C. (1993) "Mechanisms of particle transport in process equipment," J. Electrochem. Soc. 140(10): 2917-2922.

22. Eglin, J.M. (1923) "The coefficients of viscosity and slip of carbon dioxide by the oil drop method and the law of motion of an oil drop in carbon dioxide, oxygen, and helium, at low pressures," Phys. Rev. 22: 161-170.

23. Epstein, P.S. (1924) "On the resistance experienced by spheres in their motion through gases", Phys. Rev. 23: 710-733.

24. Evans, G.H. and Greif, R. (1987a) "A numerical model of the flow and heat transfer in a rotating disk chemical vapor deposition reactor," J. Heat Transfer 109: 928-935.

25. Evans, G.H. and Greif, R. (1987b) "Effects of boundary conditions on the flow and heat transfer in a rotating disk chemical vapor deposition reactor," Numerical Heat Transfer 12: 243-252.

26. Evans, G.H. and Greif, R. (1988) "Forced flow near a heated rotating disk: a similarity solutions," Numerical Heat Transfer 14: 373-387.

27. Friedlander, S.K. (1977) Smoke, Dust and Haze, J. Wiley and Sons, New York.

28. Fuchs, N.A. (1964) The Mechanics of Aerosols, Dover Publications, New York.

29. Henderson, C.B. (1976) "Drag coefficients of spheres in continuum and rarefied flows," ALAA J. 14(6): 707-708.

30. Hinds, W.C. (1982) Aerosol Technology, Wiley, New York.

31. Houtman, C., Graves, D.B., and Jensen, K.F. (1986) "CVD in stagnation point flow: an evaluation of the classical 1D treatment," J. Electrochem. Soc. 133(5): 961-970.

32. Ishida, Y. (1923) "Determination of viscosities and of the Stokes-Millikan Law constant by the oil drop method," Phys. Rev. 21: 550-563.

33. Jellum, G.M., Daugherty, J.E., and Graves, D.B. (1991) "Particle thermophoresis in low pressure glow discharges," J. Appl. Phys. 69: 6923-6934

34. Knudsen, M. and Weber, S. (1911) Ann. Phys. 36: 981-994.

35. Levich, V.G. (1962) Physicochemical Hydrodynamics Prentice-Hall, Englewood Cliffs, NJ.

36. Liu, B.Y.H. and Ahn, K-h. (1987) "Particle deposition on semiconductor wafers," Aerosol Sci. Technol. 6: $215-224$

37. Marple, V.A. and Liu, B.Y.H. (1974) "Characteristics of laminar jet impactors," Environ. Sci. Technol. 8(7): 648-654.

38. Marple, V.A. and Liu, B.Y.H. (1975) “On fluid flow and aerosol impaction in inertial impactors," J. Colloid Interface Sci. 53(1): 31-34. 
39. Millikan, R.A. (1923) "The general law of fall of a small spherical body through a gas, and its bearing upon the nature of molecular reflection from surfaces," Phys. Rev. 22: 1.

40. Opiolka, S., Schmidt, F., and Fissan, H. (1994) "Combined effects of electrophoresis and thermophoresis on particle deposition onto flat surfaces," J. Aerosol Sci. 25(4): 665-671.

41. Otani, Y., Emi, H., Kanaoka, C., and Kato, K. (1989) "Determination of deposition velocity onto a wafer for particles in the size range between 0.03 and $0.8 \mu \mathrm{m},{ }^{\prime}$ J. Aerosol Sci. 20(70): 787-796.

42. Periasamy, R., Yamamoto, T., Donovan, R.P., and Clayton, A.C. (1993) "Particle transport at subatmospheric pressures: models and verification," J. Electrochem. Soc. 140(10): 2949-2951.

43. Peters, M.H. and Cooper, D.W. (1991) "Approximate analytic solutions for particle deposition in viscous stagnation-point flow in the inertial-diffusion regime with external forces," J. Colloid and Interface Sci. 142(1): 140-148.

44. Peters, M.H., Cooper, D.W., and Miller, R.J. (1989) "The effects of electrostatic and inertial forces on the diffusive deposition of small particles onto large disks: viscous axisymmetric stagnation point flow approximations," J. Aerosol Sci. 20(1): 123-136.

45. Peterson, T.W., Stratmann, F., and Fissan, H. (1989) "Particle deposition on wafers: a comparison between two modeling approaches," J. Aerosol Sci. 20(6): 683-693.

46. Pui, D.Y.H., Ye, Y., and Liu, B.Y.H. (1990) "Experimental Study of Particle Deposition on Semiconductor Wafers," Aerosol Sci. Technol. 12: 795-804.

47. Rader, D.J. (1990) "Momentum slip correction factor for small particles in nine common gases," $J$. Aerosol Sci. 21(2): 161-168.

48. Rader, D.J. and Geller, A.S. (1994) "Particle transport modeling in semiconductor process environments," Plasma Sources Sci. Technol. 3: 426-432.

49. Rader, D.J. and Geller, A.S. (1998) "Showerhead-enhanced inertial particle deposition in parallelplate reactors," Aerosol Sci. Technol. 28: 105-132.

50. Rader, D.J., Geller, A.S., and Choi, S.J. (1999) "Particle deposition in parallel plate reactors I. Simultaneous diffusion and external forces," submitted to Aerosol Sci. Technol.

51. Rader, D.J., Geller, A.S., Choi, S.J., and Kushner, M.J. (1994a) "Application of numerical models to predict particle contamination in semiconductor process environments," 1994 Proceedings Institute of Environmental Sciences 40th Technical Meeting: 308-315.

52. Rader, D.J., Geller, A.S., Choi, S.J., and Kushner, M.J. (1994b) "Particle transport in plasma reactors," Microcontamination 94 Conference Proc., Oct. 4-6, San Jose, CA: 39-48.

53. Rader, D.J. and Marple, V.A. (1985) "Effect of ultra-Stokesian drag and particle interception on impaction characteristics," Aerosol Sci. Technol. 4: 141-156.

54. Ramarao, B.V., and Tien, C. (1989) "Aerosol deposition in two-dimensional laminar stagnation flow," J. Aerosol Sci. 20(7): 775-785.

55. Robinson, A. (1956) "On the motion of samll particles in a potential field of flow," Communications on Pure and Applied Mathematics 9:69-48

56. Roth, R. M., Spears, K. G., Stein, G. D., and Wong, G. (1985) "Spatial dependence of particle light scattering in an rf silane discharge," Appl. Phys. Lett. 46: 253-255.

57. Schmidt, F., Stratmann, F., Fissan, H., Periasamy, R., Donovan, R.P., Clayton, A.C., Yamamoto, T., and Ensor, D.S. (1992) "Theoretical and experimental study of particle transport in vacuum chambers," J. Aerosol Sci. 23(S1): 59-62. 
58. Shiratani, M., Matsuo, S., and Watanabe, Y. (1991) "In Situ Observation of Particle Behavior in rf Silane Plasmas," Jpn. J. Appl. Phys. 30: 1887.

59. Sommerer, T. J., Barnes, M. S., Keller, J. H., McCaughey, M. J., and Kushner, M. J. (1991) “Monte Carlo-fluid hybrid model of the accumulation of dust particles at sheath edges in radio-frequency discharges," Appl. Phys. Lett. 59(6): 638-640.

60. Stratmann, F., Fissan, H., and Peterson, T.W. (1988) "Particle deposition onto a flat surface from a point particle source," J. Environ. Sci. 31: 39-41.

61. Talbot, L., Cheng, R.K., Schefer, R.W., and Willis, D.R. (1980) "Thermophoresis of particles in a heated boundary layer," J. Fluid Mech. 101(4): 737-758.

62. Terrill, R.M. and Cornish, J.P. (1973) "Radial flow of a viscous, incompressible fluid between two stationary, uniformly porous discs," J. Appl. Math. Phys. (ZAMP) 24: 676-688.

63. Turton, R. and Levenspiel, O. (1986) "A short note on the drag correlation for spheres," Powder Technol. 47: 83-86.

64. Waldmann, L. and Schmitt, K.H. (1966) "Chapter VI. Thermophoresis and Diffusiophoresis of Aerosols," in Aerosol Science, C.N. Davies, ed., Academic Press, New York.

65. Wu, J.J.,Miller, R.J, Cooper, D.W., Flynn, J.F., Delson, D.J., and Teagle, R.F. (1989) "Deposition of submicron aerosol particles during integrated circuit manufacturing: Experiments," J. Environ. Sci. 32(1): 27-28,43-45.

66. Ye, Y., Pui, D.Y.H., Liu, B.Y.H., Opiolka, S., Blumhorst, S., and Fissan, H. (1991) “Thermophoretic Effect of Particle Deposition on a Free Standing Semiconductor Wafer in a Clean Room," J. Aerosol Sci. 22(1): 63-72.

67. Yeon, C.-K., Kim, J.-h., and Whang, K.-W. (1995) "Dynamics of particulates in the afterglow of a radio frequency excited plasma,” J. Vac. Sci Technol. A. 13: 927-930.

68. Yuu, S. and Jotaki, T. (1978) "The calculation of particle deposition efficiency due to inertia, diffusion and interception in a plane stagnation flow," Chem. Eng. Sci. 33: 971-978. 


\section{Appendix}

Table 3: Tabulation of Grand Design Curve Data. Critical Stokes numbers for various dimensionless jet velocities and showerhead thicknesses (Re $=0$, no external force applied, $r_{p} / S=10^{-7}$ )

\begin{tabular}{|c||c|c|c|c|c|c|c|}
\cline { 2 - 8 } \multicolumn{1}{c||}{} & \multicolumn{7}{c|}{$L / S$} \\
\hline$U_{\text {jet }} / U_{0}$ & 0.05 & 0.1 & 0.2 & 0.5 & 1.0 & 2.0 & 5.0 \\
\hline \hline 0.1 & .4312 & .4403 & .4549 & .4742 & .4753 & .4753 & .4753 \\
\hline 0.2 & .4301 & .4381 & .4513 & .4707 & .4739 & .4739 & .4739 \\
\hline 0.5 & .4267 & .4317 & .4401 & .4546 & .4614 & .4624 & .4624 \\
\hline 1.0 & .4210 & .4210 & .4210 & .4210 & .4210 & .4210 & .4210 \\
\hline 1.4 & .4165 & .4125 & .4059 & .3932 & .3842 & .3799 & .3791 \\
\hline 2.0 & .4098 & .4000 & .3839 & .3544 & .3339 & .3232 & .3208 \\
\hline 3.2 & .3966 & .3760 & .3440 & .2914 & .2592 & .2424 & .2378 \\
\hline 5.0 & .3776 & .3432 & .2947 & .2270 & .1912 & .1736 & .1685 \\
\hline 7.0 & .3578 & .3114 & .2524 & .1810 & .1473 & .1314 & .1266 \\
\hline 10. & .3306 & .2720 & .2065 & .1382 & .1093 & .09606 & .09199 \\
\hline 14. & .2991 & .2314 & .1654 & .1049 & .08116 & .07064 & .06736 \\
\hline 20. & .2602 & .1881 & .1268 & .07685 & .05852 & .05054 & .04804 \\
\hline 32. & .2048 & .1358 & .08615 & .05004 & .03754 & .03219 & .03051 \\
\hline 50. & .1539 & .09538 & .05805 & .03283 & .02441 & .02084 & .01972 \\
\hline 100. & .09011 & .05198 & .03042 & .01679 & .01238 & .01053 & .009941 \\
\hline 200. & .04899 & .02716 & .01557 & .008485 & .006232 & .005290 & .004992 \\
\hline
\end{tabular}




\section{Distribution}

$\begin{array}{lll}\text { MS 0874 } & 1705 & \text { R.S. Blewer } \\ \text { MS 0874 } & 1705 & \text { R.P. Donovan } \\ \text { MS 9409 } & 8270 & \text { L.E. Klebanoff } \\ \text { MS 0841 } & 9100 & \text { P. J. Hommert } \\ \text { MS 0828 } & 9103 & \text { T. C. Bickel } \\ \text { MS 0827 } & 9114 & \text { R.O. Griffith } \\ \text { MS 0827 } & 9114 & \text { J.E. Brockmann } \\ \text { MS 0827 } & 9114 & \text { S.J. Choi } \\ \text { MS 0827 } & 9114 & \text { A.S. Geller (10) } \\ \text { MS 0827 } & 9114 & \text { D.J. Rader (10) } \\ \text { MS 9018 } & 8940-2 & \text { Central Technical Files } \\ \text { MS 0899 } & 4916 & \text { Technical Library (2) } \\ \text { MS 0619 } & 00111 & \text { Review and Approval Desk }\end{array}$

David Graves

University of California, Berkeley

Department of Chemical Engineering

Berkeley, CA 94720-1462

Mark Kushner

University of Illinois

Department of Electrical and Computer Eng.

1406 W. Green St.

Urbana, IL 61801

Peter McMurry

University of Minnesota

125 Mechanical Engineering

111 Church St. SE

Minneapolis, MN 55455

SEMATECH (4)

David Jensen

Brad Van Eck

Mark Louis

Robin Worley

2706 Montopolis Dr.

Austin, TX 78741
Pat Gabella

Semi/SEMATECH

2706 Montopolis Dr.

Austin, TX 78741

Applied Materials (3)

Dan Clark

Terry Francis

Sandeep Nijhawan

3050 Bowers Ave.

Santa Clara, CA 95054

FSI International (2)

Scott Becker

Natraj Narayanswami

322 Lake Hazeltine $\mathrm{Dr}$

Chaska, MN 55318

Dikran Babikian

Silicon Valley Group, Inc.

2240 Ringwood Ave.

San Jose, CA 95131

Novellus (2)

Ed Baker

Don Schlosser

M/S 350

3970 N. First St.

San Jose, CA 95134

Douglas Brown

Varian

35 Dory Rd.

Gloucester, MA 01930

Iraj Emami

AMD

5204 East Ben White Blvd

M/S 608

Austin, TX 78741-7306

Intel (2)

Scott Sibbett

Randy Williams

4100 Sara Road

Rio Rancho, NM 87124 
Sadasivan Shankar

Intel

RN2-40

2200 Mission College Blvd.

P.O. Box 58119

Santa Clara, CA 95052-8119

Texas Instruments (5)

Allen Bowling

J. Michael Grobelny

Asad Haider

Venu Menon

Thomas Schneider

13353 Floyd Rd.

Dallas, TX 75265

Susan L. Cohen

IBM

11-253

IBM T.J. Watson Research Center

Yorktown Heights, NY 10598 